Aus der Abteilung Allgemeinmedizin

(Kommissarische Leiterin Dr. med. Anne Simmenroth-Nayda)

im Zentrum Innere Medizin

der Medizinischen Fakultät der Universität Göttingen

und dem Institut für Allgemeinmedizin

(Prof. Dr. med. Martin Scherer)

Universitätsklinikum Hamburg-Eppendorf

\title{
Veränderungen von Parametern der Sprechstimme
}

im

\section{Medizinstudium}

(Kurztitel ,Stimmstudie')

\author{
INAUGURAL - DISSERTATION \\ zur Erlangung des Doktorgrades \\ der Medizinischen Fakultät \\ der Georg-August-Universität zu Göttingen
}

vorgelegt von

Maria Rodenstock geb. Strauß

aus

Leinefelde

Göttingen 2012 
D e k a n: $\quad$ Prof. Dr. med. C. Frömmel

I. Berichterstatter: Prof. Dr. med. M. Scherer

II. Berichterstatter/in:

III. Berichterstatter/in:

Tag der mündlichen Prüfung: 


\section{Inhaltsverzeichnis}

ABKÜRZUNGSVERZEICHNIS ...............................................................................

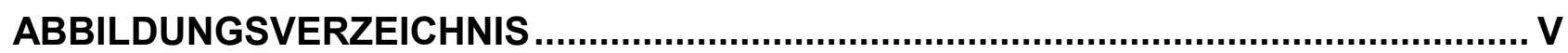

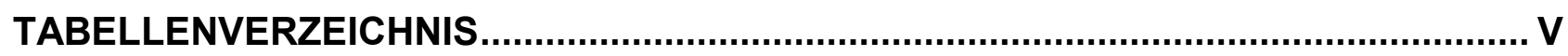

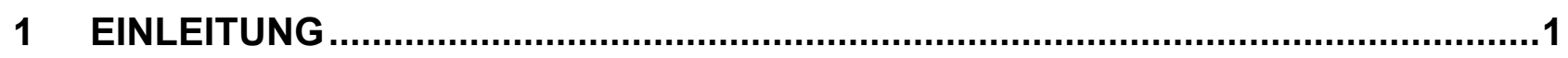

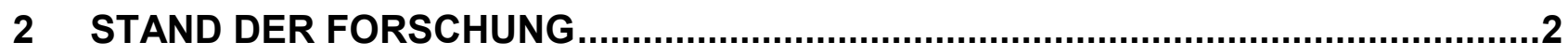

2.1 Stimme

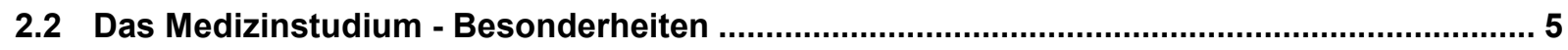

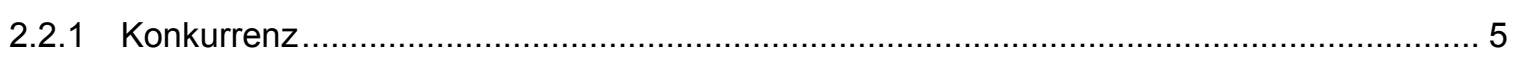

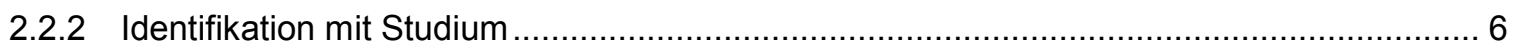

2.2.3 Medizinische Kommunikation und professionelle Identität ............................................... 7

2.3 Die Kommunikation des Arztes und ihre Wirkung auf Patienten ....................................... 8

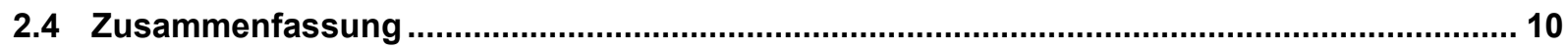

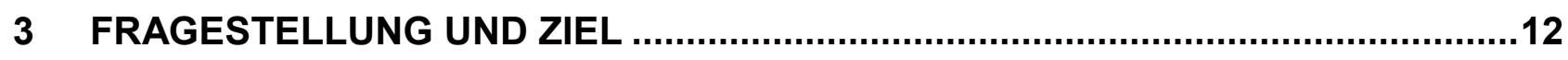

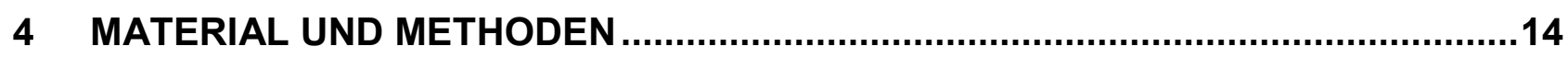

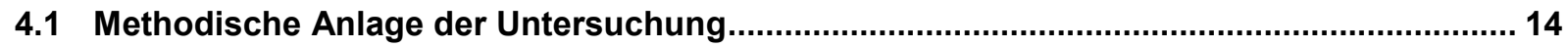

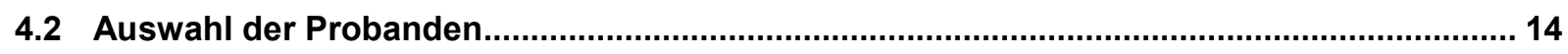

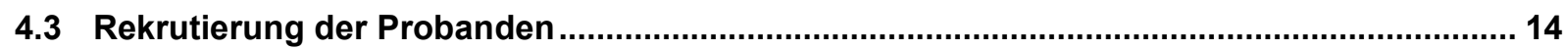

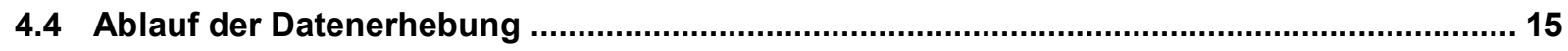

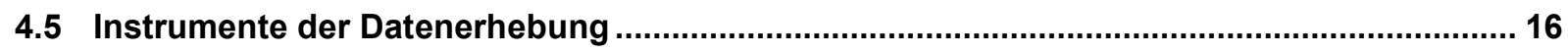

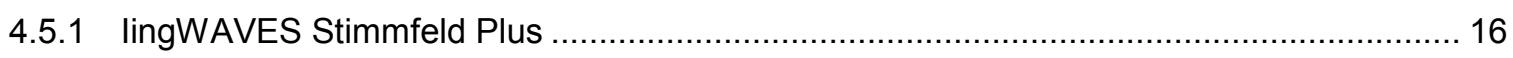

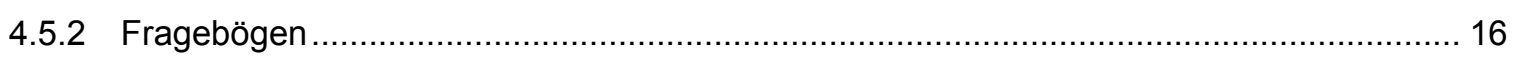

4.5.2.1 lingWAVES Fragebogen VDI-Stimmstörungsindex ....................................... 17

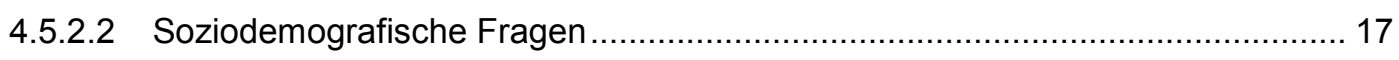

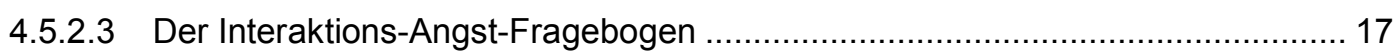

4.5.2.4 Das Freiburger Persönlichkeitsinventar ..................................................... 18 


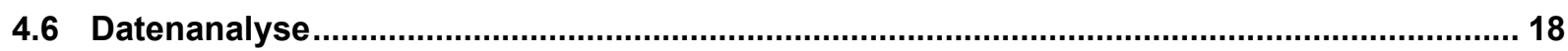

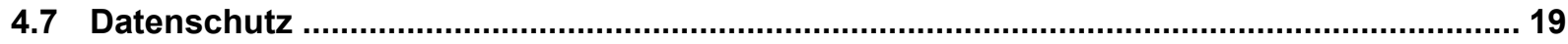

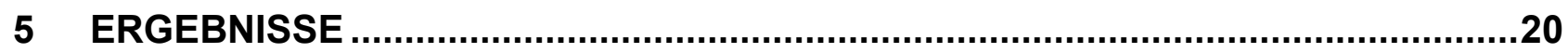

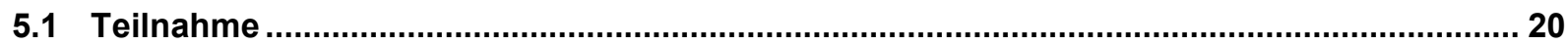

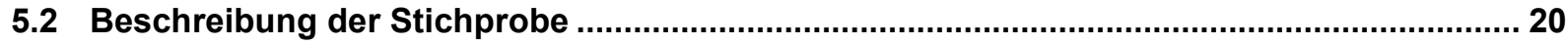

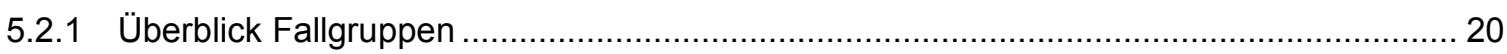

5.2.2 Vergleich der Fallgruppen bezüglich soziodemografischer Daten ............................ 22

5.2.3 Einteilung des Studienkollektivs nach Stimmlautstärke................................... 23

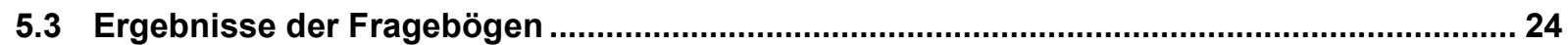

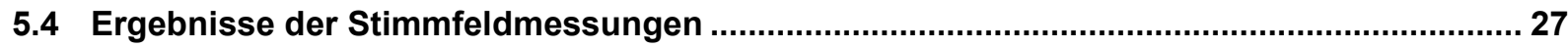

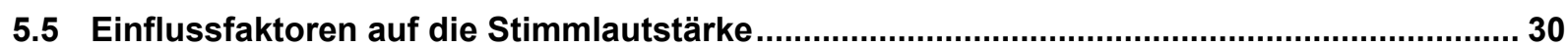

6 DISKUSSION

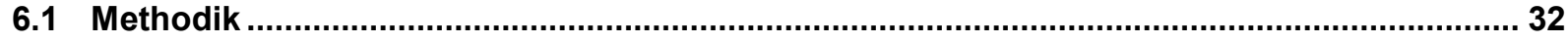

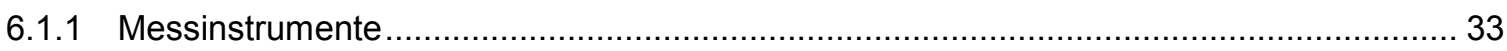

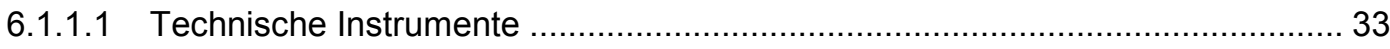

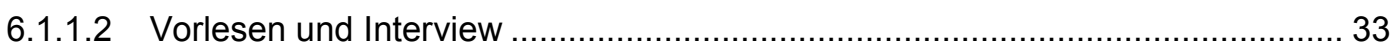

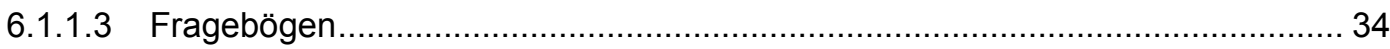

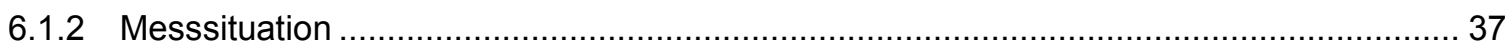

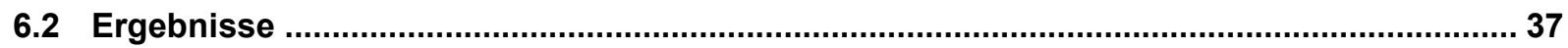

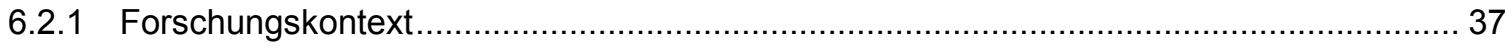

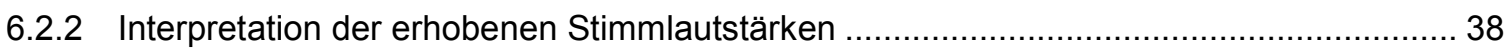

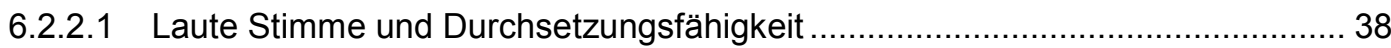

6.2.2.2 Laute Stimme und Selbstvertrauen ...................................................... 39

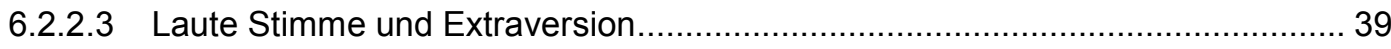

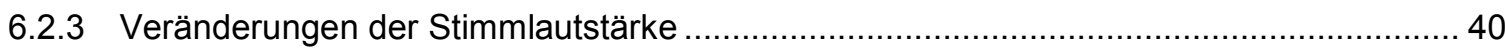

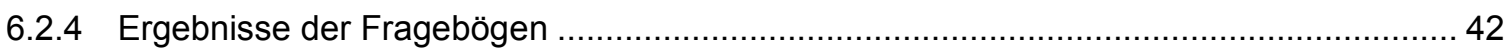

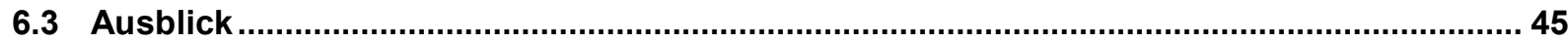

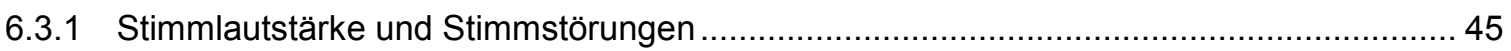




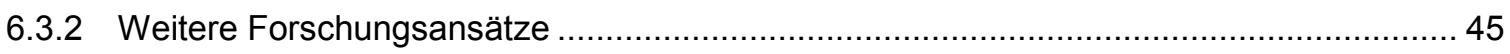

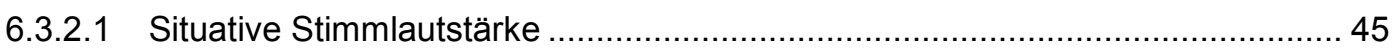

6.3.2.2 Stimme in verschiedenen ärztlichen Positionen ................................................ 46

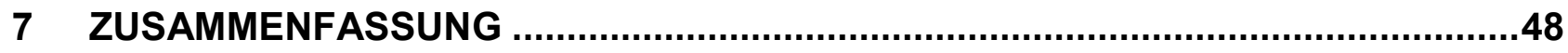

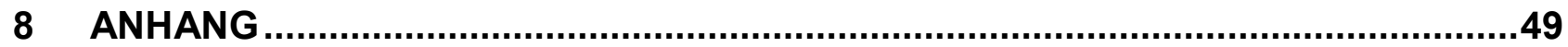

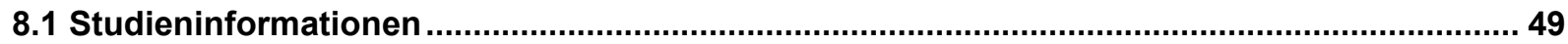

8.2 Aushang

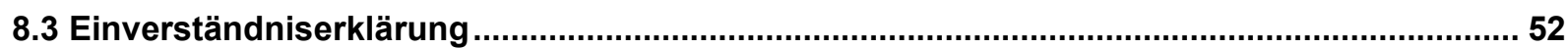

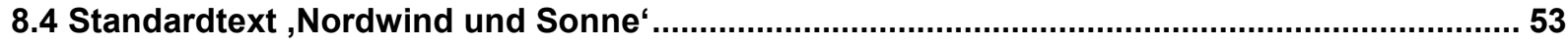

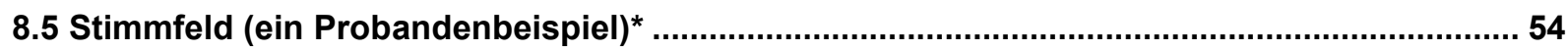

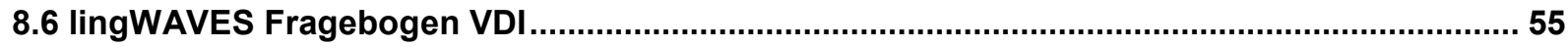

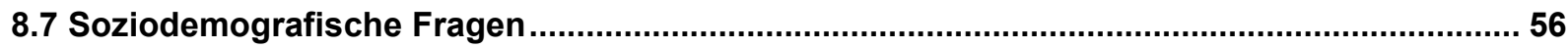

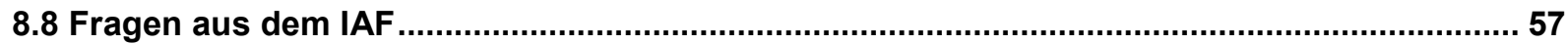

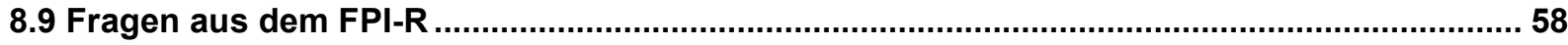

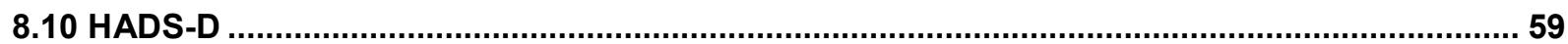

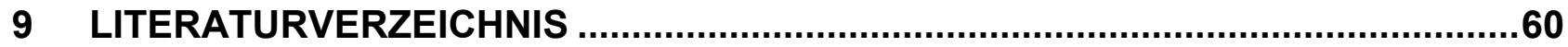




\section{Abkürzungsverzeichnis}

\begin{tabular}{|l|l|}
\hline Abkürzung & Erläuterung \\
\hline Abb. & Abbildung \\
\hline bzw. & beziehungsweise \\
\hline dB & Dezibel \\
\hline et al. & et alii \\
\hline etc. & et cetera \\
\hline FRI-R & Freiburger Persönlichkeitsinventar - Revision \\
\hline HADS-D & $\begin{array}{l}\text { Hospital Anxiety and Depression Scale - Deutsche } \\
\text { Version }\end{array}$ \\
\hline HADS-D/A & $\begin{array}{l}\text { Angstskala der Hospital Anxiety and Depression } \\
\text { Scale - Deutsche Version }\end{array}$ \\
\hline HADS-D/D & $\begin{array}{l}\text { Depressionsskala der Hospital Anxiety and } \\
\text { Depression Scale - Deutsche Version }\end{array}$ \\
\hline IAF & Interaktions - Angst - Fragebogen \\
\hline Kap. & Kapitel \\
\hline Med. & Mediziner \\
\hline SD & standard deviation (Standardabweichung) \\
\hline Sem. & Semester \\
\hline sonst. & Sonstiges \\
\hline SSI & Stimmstörungsindex \\
\hline u.a. & unter anderem \\
\hline VDI & Voice Disorder Index \\
\hline Vgl. & Vergleich \\
\hline
\end{tabular}




\section{Abbildungsverzeichnis}

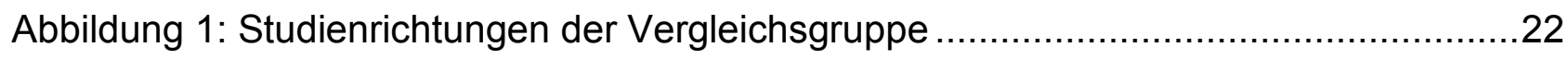

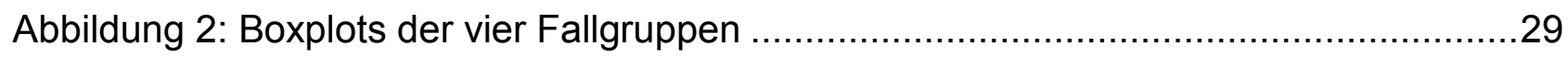

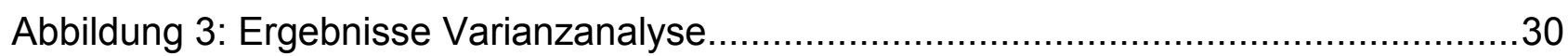

\section{Tabellenverzeichnis}

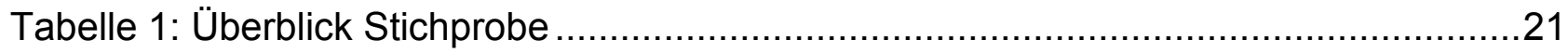

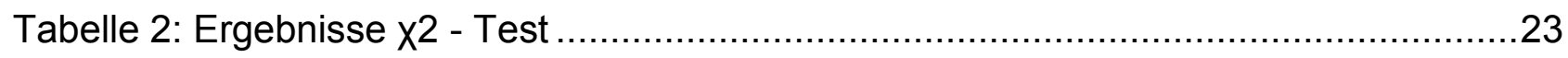

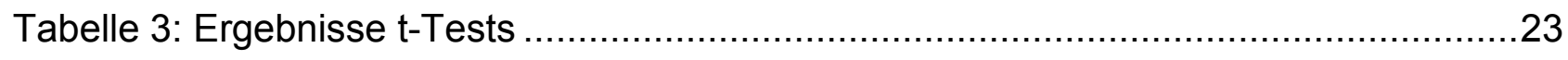

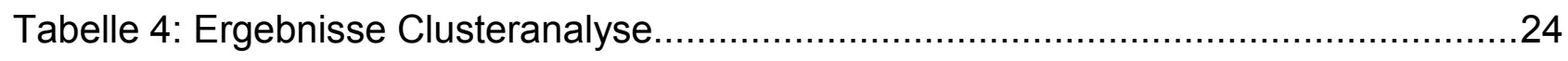

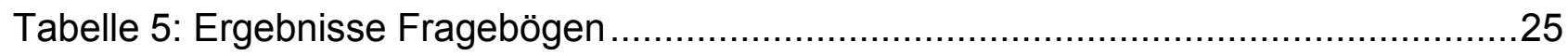

Tabelle 6: Mittelwerte Stimmfeldmessungen ……...................................................28 


\section{Vorwort}

„Die Zukunft des Arztes. - Es gibt jetzt keinen Beruf, der eine so hohe Steigerung zuließe, wie der des Arztes; namentlich nachdem die geistlichen Ärzte, die sogenannten Seelsorger ihre Beschwörungskünste nicht mehr unter öffentlichem Beifall treiben dürfen und ein Gebildeter innen aus dem Wege geht. Die höchste geistige Ausbildung eines Arztes ist jetzt nicht erreicht, wenn er die besten neuesten Methoden kennt und auf sie eingeübt ist und jene fliegenden Schlüsse von Wirkungen auf Ursachen zu machen versteht, derentwegen die Diagnostiker berühmt sind: er muss außerdem eine Beredsamkeit haben, die sich jedem Individuum anpasst und inm das Herz aus dem Leibe zieht, eine Männlichkeit, deren Anblick schon den Kleinmut (den Wurmfrass aller Kranken) verscheucht, eine Diplomaten-Geschmeidigkeit im Vermitteln zwischen Solchen, welche Freude zu ihrer Genesung nötig haben und Solchen, die aus Gesundheitsgründen Freude machen müssen (und können), die Feinheit eines Polizeiagenten und Advokaten, die Geheimnisse einer Seele zu verstehen, ohne sie zu verraten, - kurz ein guter Arzt bedarf jetzt der Kunstgriffe und Kunstvorrechte aller andern Berufsklassen: so ausgerüstet, ist er dann im Stande, der ganzen Gesellschaft ein Wohltäter zu werden, durch Vermehrung guter Werke, geistiger Freude und Fruchtbarkeit, durch Verhütung von bösen Gedanken, Vorsätzen, Schurkereien (deren ekler Quell so häufig der Unterleib ist), durch Herstellung einer geistig-leiblichen Aristokratie (als Ehestifter und Eheverhinderer), durch wohlwollende Abschneidung aller sogenannten Seelenqualen und Gewissensbisse: so erst wird er aus einem "Medizinmann" ein Heiland und braucht doch keine Wunder zu tun, hat auch nicht nötig, sich kreuzigen zu lassen." (Nietzsche 1878/1999, S. 203) 


\section{Einleitung}

Es ist bekannt, dass sich Medizinstudenten während der Universitätszeit ein umfangreiches Wissen aneignen. Neben dem Lernzuwachs streben die jungen Mediziner nach einer kontinuierlichen Weiterentwicklung ihrer eigenen Persönlichkeit (Leo and Eagen 2008). Sie erkennen, welche Fähigkeiten, Charakterzüge und Verhaltensweisen innerhalb ihrer Fakultät wertgeschätzt werden. Sie nehmen wahr und interpretieren, was Lehrende und Patienten von ihnen erwarten. In den zukünftigen Arztberuf werden hohe gesellschaftliche Ideale projiziert (Plewnia 1999). Diesen Erwartungen versuchen Studierende der Medizin gerecht zu werden.

Das Medizinstudium trainiert die angehenden Ärzte für die zukünftige Berufsrolle im Sinne einer professionellen Dominanz, soziologisch mit dem Terminus, professionelle Identität' beschrieben (u.a. Lingard et al. 2003, Dornan and Bundy 2004). Dies geschieht vorrangig im ,hidden curriculum‘. Der ,heimliche Lehrplan“ wird praktisch nie offen diskutiert oder reflektiert (Hafferty 1998).

Eine Möglichkeit, Patienten, Pflegepersonal oder Kollegen zu erreichen, sie zu beeinflussen, gar zu leiten, stellt das Arbeitsinstrument ,Stimme‘ dar. Die Sprechstimme ist durch verschiedene akustische Parameter festgelegt und wird hörbar durch Veränderungen in der Tonhöhe und Lautstärke moduliert.

Eisler et al. (1973) zeigten, dass überzeugendes bzw. durchsetzungsfähiges Verhalten (,assertive behaviour“) mit einem lauteren Sprechen assoziiert ist. Durch Lautstärke kann dem Gesagten Nachdruck verliehen werden und somit wird der Eindruck von Dominanz vermittelt. Lautes und schnelles Sprechen werten Menschen als beeindruckend und überzeugend. „Lautstärke demonstriert ein starkes Selbstbewusstsein und erhöht die Autorität" (Prost 2009, S. 185).

Während ihres Studiums arbeiten Mediziner daran, sich professionelle Identität und Selbstbewusstsein anzueignen (Dornan and Bundy 2004). Diese Anforderungen sind Bestandteil der zukünftigen Arztrolle. Da eine laute Stimme als charakteristisches Merkmal selbstbewusster Menschen gilt, ist zu vermuten, dass bei Medizinstudenten die Zunahme der Sprechstimmenlautstärke größer ist als bei Studierenden anderer Fachrichtungen. Sollten sich die Erwartungen bestätigen, könnten professionelle Sozialisationsprozesse im Medizinstudium besser verstanden werden. Bisher gibt es zur Veränderung der Sprechstimmenlautstärke im Studium nur wenige Daten. In der vorliegenden Querschnittsstudie (quasi-longitudinal) wird der Frage nachgegangen, ob die Lautstärke der Sprechstimme von Medizinstudenten im Verlauf ihres Studiums ansteigt. 


\section{Stand der Forschung}

\subsection{Stimme}

Die menschliche Stimme ist das hörbare Ergebnis von Schallwellen, welche individuell von unseren Stimmorganen modifiziert werden. Die Stimmbildung (Phonation) stellt einen komplexen Prozess dar, an dem verschiedene Funktionsbereiche des menschlichen Körpers beteiligt sind. Dazu zählen der Kehlkopf, der Atemapparat, die sogenannten Ansatz- und Resonanzräume sowie zentralnervöse Strukturen. Zur Stimmerzeugung wird aerodynamische Energie in akustische Energie umgewandelt (Wendler et al. 2005). Dies geschieht, indem ausgeatmete Luft beim Vorbeiströmen an den Stimmlippen im Kehlkopf in Schwingungen versetzt wird. Dabei liegen die Stimmbänder eng nebeneinander und formen einen schmalen Spalt (Phonationsstellung). Die Lautstärke der Stimme wird erzeugt durch die Amplitude der Schwingungen, wogegen die Schwingungsfrequenz die Tonhöhe bestimmt (Spornitz 2004). In dem Bereich oberhalb der Stimmlippen liegen die sogenannten Ansatzräume, die sich aus Rachen-, Mund- und Nasenraum zusammensetzen. In den Ansatzräumen wird die vorbeiströmende Luft so beeinflusst und gelenkt, dass Sprachlaute entstehen. Diesen Prozess bezeichnet man definitionsgemäß als Artikulation (Grassegger 2010). Stimmlaute werden verstärkt in Resonanzräumen. „Diesem Zweck dienen die vielen kleinen Hohlräume in Knochen, wie beispielsweise die Kiefer-, Stirn- und Keilbeinhöhlen, und die größeren Hohlräume wie der Rachen, die Nasenhöhle und die Mundhöhle sowie der Brustkorb" (Gühring und Barth 1992, S. 229). Die verschiedenen Funktionsbereiche zur Stimmbildung wie Kehlkopf, Ansatz- und Resonanzräume weisen individuelle anatomische Gegebenheiten auf, so dass die anatomisch-physischen Voraussetzungen eines Menschen Einfluss auf die Stimmbildung nehmen.

Durch unsere Stimme teilen wir uns den Mitmenschen mit, wir nutzen sie als Mittel zur Kommunikation. „In der verbalen Kommunikation dient Sprache als Informationsträger" (Philipp 2004, S. 343). Die verbale Kommunikation setzt sich aus linguistischen und paralinguistischen Aspekten zusammen. Zu den linguistischen Aspekten gehören Vokabular, Grammatik und Syntax. Quantitativ umfangreicher gestalten sich die paralinguistischen Aspekte der verbalen Kommunikation. Dazu zählt man die prosodischen Merkmale Stimmlautstärke, Sprechrhythmus, Stimmhöhe beziehungsweise Stimmtiefe (aus dem Wechsel von Stimmhöhe und Stimmtiefe ergibt sich die Stimmmelodie), Akzent, Sprechgeschwindigkeit und den Einsatz von Pausen (Piok 2011). Zur Vollständigkeit sollen die nonverbalen Kommunikationsmittel hier erwähnt werden. Nonverbale Kommunikation findet ohne Sprache statt, zum Beispiel durch Blickverhalten, Mimik und Gestik (Bründler et al. 2004). 
Ursprünglich und gewöhnlich gebrauchen wir unsere Stimme, um Dialoge zu führen. Dabei ist der Dialog an sich ohne besondere Spannung, wenn keine speziellen Ziele durchzusetzen sind. Auf einer Stufe höherer Intensität gibt es dann eine bewusste und gespannte Stimmführung dem gegebenen Anlass entsprechend. So beobachten lässt sich das bei einer Unterhaltung mit im vorab geplantem Ziel, sehr eindrucksvoll zum Beispiel in Debatten zwischen Politikern. Aber auch Arzt-Patienten-Gespräche beinhalten häufig vorgefasste Ziele, beispielsweise die Intention des Arztes, den Patienten von der Richtigkeit seiner Diagnose oder einer bestimmten Therapie zu überzeugen. Vergleichbare Gesprächsziele gibt es auch unter ärztlichen Kollegen.

In der Stimmführung spiegeln sich diese vorgefassten Ziele wider, denn die Wirkung der Kommunikation lässt sich gerade durch die Stimme und ihrer Parameter modulieren. Durch ihren Einsatz wird die gesendete Botschaft abgeschwächt oder verstärkt. Um die Suggestivkraft des Gesagten zu erhöhen, stehen dem Individuum verschiedene Möglichkeiten zur Verfügung. Die Änderung der Sprechstimmenlautstärke stellt unter anderem eine dieser Möglichkeiten dar.

Wie bereits dargelegt, ist die Stimmlautstärke ein paralinguistisches Merkmal der verbalen Kommunikation (Piok 2011). Die Paralinguistik wird definiert durch ihren Ausdruck von Emotionen und Affekten des Sprechers (Grimm und Engelkamp 1981). „Über situationsbedingte Gefühle und Stimmungen hinaus, zeigt die Stimme, wie eine Person ist und welche Eigenschaften sie hat" (Bazil und Piwinger 2009). Dabei lässt insbesondere die Lautstärke den Worten eine Bedeutung zukommen, die über den Informationsgehalt des Gesagten, also über die verbale Kommunikation, hinausgeht.

Anhand der Bandbreite der Lautstärke lässt sich die Steigerungsfähigkeit der Stimme erkennen. Um Stimmlautstärken zu vergleichen, bedarf es objektiver und quantitativer Untersuchungen. Quantitative Untersuchungen der menschlichen Stimme erstellen sogenannte Stimmfelder - auch Phonetogramme genannt -, mit denen individuell mittlere Sprechstimmenintensitäten ermittelt werden können (Nawka et al. 2006). Die mittleren Stimmintensitäten repräsentieren die durchschnittliche Sprechstimmenlautstärke in der Maßeinheit Dezibel. Untersuchungen ergaben einen geschlechtsspezifischen Unterschied der Sprechstimmenlautstärke. Demnach reden Männer mit einer durchschnittlichen mittleren Stimmintensität von $66,1 \mathrm{~dB}$ bei einer Standardabweichung von $3,6 \mathrm{~dB}$ und Frauen mit einer durchschnittlichen mittleren Stimmintensität von $65,1 \mathrm{~dB}$ bei einer Standardabweichung von 3,6dB (Pérez Álvarez und Hacki 2000). „Die logarithmische Natur der Dezibelskala bewirkt, dass eine Zunahme um ca. $10 \mathrm{~dB}$ etwa eine Verdopplung der Lautstärkewahrnehmung darstellt“ (Hosse 2008, S. 2434). Die menschliche Stimme unterliegt zudem Alterungsprozessen. Ramig et al. beschreiben, dass eine alternde Stimme an Lautstärke abnimmt (Ramig et al. 2001). Eine normale Unterhaltung wird bei einer mittleren Lautstärke von $65 \mathrm{~dB}$ 
geführt (Schultz-Coulon und Asche 1988). Durch den Einsatz einer erhöhten Sprechstimmenlautstärke versuchen Menschen, andere auf sich aufmerksam zu machen, sie zu überzeugen und zu führen. Hinter dem Begriff ,führen' verbergen sich hierbei Ansprüche wie ,Orientierung geben' oder auch ,bestimmen'. Außerdem gilt lautes Sprechen als typisches Merkmal selbstbewusster Menschen. Eine laute Stimme klingt immer überzeugender als akustische Dissonanz. Zurückhaltende und scheue Menschen hingegen sprechen leise. „Zu leise Stimmen wirken unsicher“ (Amon 2008, S. 148). Will man sich in seinem Sozialisationsumfeld behaupten, ist es angebracht, sich ,Gehör zu verschaffen:.

Übertragen lässt sich das auch auf Medizinstudenten:

Medizinstudenten halten Referate, sie stellen Fragen und antworten Dozenten in Hörsälen, die von bis zu 300 Kommilitonen gefüllt werden. Sie sprechen mit schwerhörigen Patienten, sie müssen in Visiten und Besprechungen Patienten vorstellen. All diesen kommunikativen Herausforderungen kann man sich nur erfolgreich stellen, wenn man lernt, laut und deutlich zu reden. Studierende der Medizin wissen um diesen Aspekt, sehen aber gerade in der paralinguistischen Kommunikation ihre Schwächen (Zick et al. 2007).

Zwischen den Jahren 2000 und 2003 führte das ,Center for Communication and Medicine' der Northwestern University Feinberg in Chicago eine repräsentative Studie durch, um Kommunikationsfähigkeiten von Medizinstudenten zu bewerten. An dieser Studie beteiligten sich knapp 680 Medizinstudenten. Zur Durchführung des Projektes wurden diese im ersten Jahr des klinischen Studienabschnitts bei Gesprächen mit Simulationspatienten gefilmt. Danach sollten die Studenten ihre Aufnahmen in einem standardisierten Fragebogen selbst einschätzen. Ein Punkt dieses Fragebogens erfasste die paralinguistischen Kommunikationsmittel (,Paralanguage'), welche den Parameter Lautstärke implizierten. Die Auswertung der Selbsteinschätzungen ergab, dass von 674 Studenten nur 5,35\% in der paralinguistischen Kommunikation ihre Stärken sahen, wogegen $31,9 \%$ der Befragten darin ihre Schwächen angaben. Sie wünschten sich darüber hinaus eine Verbesserung der eigenen Fertigkeiten in diesem Bereich (Zick et al. 2007). Eine Verbesserung der paralinguistischen Fertigkeiten - so auch der Sprechstimmenlautstärke - verhält sich direkt proportional zur persönlichen und professionellen Entwicklung (Hays et al. 2002).

Insbesondere die zuletzt genannte Angabe ist bezogen auf die Stimmstudie von speziellem Interesse. 


\subsection{Das Medizinstudium - Besonderheiten}

\subsubsection{Konkurrenz}

Das Umfeld von Medizinstudenten stellt in vielen Bereichen besonders hohe Anforderungen an die Studierenden. Hierzu zählt zum Beispiel der Aspekt der Konkurrenz der Medizinstudenten untereinander.

Bargel und Ramm beschreiben das soziale Klima unter den Humanmedizinstudenten als „kaltes, geschäftsmäßiges, wenig förderliches Betriebsklima“ (Bargel und Ramm 1993, S.34). Zudem zeigten sie auf, dass sich die Konkurrenz unter den Medizinstudenten seit den 80er Jahren allmählich, aber beständig verschärft. Diese These lässt sich unter anderem belegen, indem man das Konstanzer Projekt ,Studiensituation' hinzuzieht. Dies ist eine Langzeitstudie zu den Themen Studentensituation und studentische Orientierung, welche seit 1983 regelmäßig im Abstand von zwei bis drei Jahren erhoben wird. Für deren Umsetzung schreibt das Bundesministerium für Bildung und Forschung bis zu 28000 Studenten verschiedener Fachrichtungen an und bittet um Studienteilnahme. Nach Auswertung der Befragungsdaten veröffentlicht das Bundesministerium für Bildung und Forschung die Studienergebnisse als allgemeine Darstellung der Hochschulsituation. Darüber hinaus sollen in Sonderauswertungen einzelne Studienrichtungen zur genaueren Betrachtung herausgegriffen werden. Dem Studium der Humanmedizin wurde 1993 als erstes in einer eigenen Veröffentlichung Beachtung geschenkt. Dies erfolgte „nicht nur wegen der Größe, Tradition und Bedeutung dieses Faches, sondern auch aus einem speziellen Grund: die Ausrichtung und das Anforderungsprofil des Studiums, [...] erscheinen in vielen und wichtigen Teilen besonders problematisch" (Bargel und Ramm 1993, S. 2). Eines der Ergebnisse dieser repräsentativen Erhebung beschreibt die Zahl an Kontakten zwischen den Studenten und den Hochschullehrern als Indikator für die Konkurrenz unter den Medizinstudenten. Das Konstanzer Projekt zeigt auf, dass nur die kleine Gruppe der Leistungsbesten einen guten Kontakt zu den Dozenten, Assistenten und Professoren hat. Daraus lässt sich ableiten, dass Studenten, die in den Vordergrund treten, bessere Chancen in ihrem Studium genießen als die Studenten, die sich zurückhalten.

Übertragen auf die vorliegende Studie ließe sich vermuten, dass auch überzeugende Kommunikation - im Sinne einer hohen Sprechstimmenlautstärke - helfen kann, sich im Studium zu behaupten. Dies könnte durch ein hohes Maß an Selbstbewusstsein, einen dominanten Stil und überzeugendes Auftreten innerhalb der Gruppe erreicht werden. Ein Mittel, um dies zu unterstreichen beziehungsweise hervorzuheben, könnte hierbei die Sprechstimmenlautstärke darstellen. 


\subsubsection{Identifikation mit Studium}

„Die Identifikation der Medizinstudierenden mit inrem Studium und der studentischen Rolle ist im Fächervergleich am höchsten" (Bargel und Ramm 1993, S. 7). Das Studium wird als "gegenwärtiger Lebensmittelpunkt" betrachtet (Bargel und Ramm 1993, S. 7). Mediziner gelten als besonders ehrgeizig. Auch diese Aussage lässt sich anhand des Konstanzer Projektes verifizieren. Mit Hilfe eines standardisierten Studentensurveys wurde aufgezeigt, dass bei den Medizinstudenten der Ehrgeiz nach einem raschen Studium und einem guten Examen deutlich stärker ist als in anderen Fächern. Medizinstudenten sind einem stetigen Leistungsdruck ausgesetzt. Die hohen Anforderungen können zu gesundheitlichen Beeinträchtigungen führen. Im Vergleich zu gleichaltrigen Bevölkerungsgruppen treten psychische Erkrankungen wie Depressionen und Burnout unter Medizinstudenten häufiger auf (u.a. Schwenk et al. 2010, Brazeau et al. 2010, Dyrbye et al. 2010). Dieser Aspekt gewinnt an Bedeutung, wenn man bedenkt, dass Menschen, die Medizin studieren, in der Gesellschaft als besonders „leistungsfähig“ und „leistungsbereit“ gelten (Bargel und Ramm 1993, S. 12).

Die Anforderungen im Medizinstudium sind hoch, die Studiengliederung streng. Es herrscht eine Arbeitskultur stark geregelter Tätigkeiten und enormer Anstrengung. Jeder Medizinstudent ist dem hohen Selektionsdruck ausgesetzt. Man sieht Kommilitonen scheitern und zwischenmenschliche Kontakte sind durch Prüfungs- und Leistungsdruck belastet. Die extreme Identifikation ist von der Medizinischen Fakultät gewünscht. Sie erleichtert es den angehenden Ärzten, sich ihre eigene professionelle Identität anzueignen.

„Grundlage ärztlichen und therapeutischen Handelns ist zunächst ein solides und umfassendes Fachwissen über Erkrankungen, deren Symptome, Ursachen und Behandlungsmöglichkeiten, das dem Patienten Sicherheit durch Professionalität vermittelt“" (Lieb et al. 2008, S. 91). Professionalität wird darüber hinaus durch Selbstdarstellung vermittelt. Um den Medizinstudenten dies aufzuzeigen, findet an vielen Universitäten weltweit die sogenannte ,Weiße-Kittel-Zeremonie‘ statt. Dies ist eine Initiative der Arnold P. Gold Foundation in England, welche bereits im ersten klinischen Semester durchgeführt wird. In einem feierlichen Rahmen tragen die Medizinstudenten erstmals ihre weißen Kittel. Sie sollen erfahren, welche Verantwortung es mit sich bringt, den weißen Kittel zu tragen. Sobald man den weißen Kittel trägt, wird Professionalität von innen erwartet. Dazu zählt auch die Fähigkeit, zu führen und zu leiten (Stern and Papadakis 2006). Die Stimme und der Gebrauch einer erhöhten Stimmlautstärke sind Bestandteil der eigenen Selbstdarstellung. Gerade in diesem Zusammenhang kann die Stimme und ihr Merkmal Lautstärke die professionelle Persönlichkeit hervorheben. 
Daher lohnen sich Studien, die messbare Parameter zwischen Medizinstudenten und Studenten anderer Fachrichtungen vergleichen. Die hier vorgelegte Stimmstudie kann einen Beitrag zum Verständnis professioneller Sozialisationsprozesse im Medizinstudium leisten.

\subsubsection{Medizinische Kommunikation und professionelle Identität}

In vielen Situationen des klinischen Alltags ist es unumgänglich, vor einer Gruppe von (oft auch fremden) Menschen zu sprechen. Diese Gruppe weist häufig eine Konstellation auf, die für den Medizinstudenten eine Herausforderung darstellt. Das wird deutlich, betrachtet man zum Beispiel eine Patientenvorstellung in der Chefarztvisite.

Lingard et al. führten 2003 eine Studie durch zum Thema „Fallvorstellung und die Entwicklung einer professionellen Identität" (Lingard et al. 2003). Die klinische Fallvorstellung stellt für junge Mediziner eine Möglichkeit dar, Wissen zu demonstrieren und Kompetenz unter Beweis zu stellen. Nach einer Zusammenarbeit mit R.J. Haber beschrieb Lingard bereits 2001 die Fallvorstellung als ein Instrument der professionellen Sozialisation (Haber and Lingard 2001). Auf dem Weg der beruflichen Sozialisation sieht sich ein Medizinstudent immer wieder mit Patientenvorstellungen konfrontiert. Er muss sich dieser Aufgabe stellen, sich darin weiterentwickeln und genug Autorität entwickeln, diese Arte der medizinischen Kommunikation zu beherrschen. Lingard et al. erkannten, dass man durch eine bestimmte Art zu reden überzeugend wirken kann. Da überzeugendes Auftreten und lautes Sprechen miteinander assoziiert sind, soll die oben genannte Studie hier vorgestellt werden.

In der Studie „Fallvorstellung und die Entwicklung einer professionellen Identität“ von L. Lingard und ihren Mitarbeitern wurden Patientenvorstellungen von Medizinstudenten im Verlauf betrachtet. Man begleitete Studenten während 3-4wöchiger Famulaturen. Die Datenerhebung erfolgte mit Hilfe von Feldbeobachtungen und standardisierten Interviews. Hierbei wurde deutlich, dass die Studenten rhetorische Fortschritte erzielten. Sie verloren zunehmend ihre Unsicherheit, der Ton der Stimme bei der Patientenvorstellung wurde bestimmter und das Auftreten selbstbewusster. Anknüpfend an diese Beobachtungen wäre es interessant, zu untersuchen, ob die Entwicklung der Studenten auch mit einer erhöhten Sprechstimmenlautstärke korreliert.

Über die rhetorischen Fortschritte hinaus wurden weitere Entwicklungen innerhalb des Studentenkollektivs dokumentiert. Diese Entwicklungen beziehen sich interessanterweise auf die Haltungen der Studenten zu den Patienten und Kollegen. Im klinischen Alltag finden sich typischerweise hierarchische Strukturen und Kollegialität kann unter Karriereorientierung leiden. Die Einstellung der Ärzte untereinander verdeutlichen Bezeichnungen wie "Unzuverlässige“ oder „Feinde“ (Lingard et al. 2003, S. 614). Das Streben nach Prestige wirkt sich dann unter Umständen auch auf die 
Haltung der Ärzte zu ihren Patienten aus: „Altruismus nimmt ab, sachlich-technisches Interesse nimmt zu (Professionalismus)" (Faller und Lang 2006, S. 160). In manchen Situationen wird der Patient mehr als Forschungsobjekt betrachtet und weniger als ein Mensch, der Zuwendung und Hilfe benötigt. Die Einstellung von Ärzten zu Patienten wird in der Literatur unter anderem bezeichnet als „paternalistisch“, „atomistisch“ oder „als Gegenstand betrachtend“" (Lingard et al. 2003, S. 614). Die Studenten in der Studie von Lingard et al. entwickelten bereits nach wenigen Wochen in der klinischen Tätigkeit Ansätze dieser Einstellungen.

Wie lässt sich das erklären? Studenten müssen in Patientenvorstellungen ihre Unsicherheit überwinden. Die Unsicherheit entsteht daraus, dass Studenten die Grenzen ihres Wissens erkennen. Trotz dieser Erkenntnis (welcher auch erfahrene Ärzte immer wieder gegenüberstehen) sehen sich Mediziner oft gezwungen, Autorität und Zuversicht zu demonstrieren - sowohl gegenüber den Patienten als auch gegenüber den Kollegen. Medizinstudenten eignen sich diese Fertigkeit an. Aus diesem Zusammenhang heraus lassen sich die oben genannten Einstellungen verstehen. Lingard et al. interpretieren diese Ergebnisse als Hinweis dafür, dass durch medizinische Rhetorik die professionelle Identität und Interaktion gefördert wird.

\subsection{Die Kommunikation des Arztes und ihre Wirkung auf Patienten}

Es gibt reine Sprechberufe, für die eine intakte Stimme Grundvoraussetzung bildet. Dazu zählen beispielsweise Lehrer, Beschäftigte in Call-Centern, Geistliche und Politiker. Ärztinnen und Ärzte bilden eine Berufsgruppe, die ihre Stimme zur Berufsausübung benötigt. Stimmstörungen können die Ausübung des Arztberufes unmöglich machen (Schneider und Bigenzahn 2007). Die Art und Weise, wie ein Arzt mit seinen Patienten spricht, hat starken Einfluss auf das Empfinden und Verhalten der jeweiligen Patienten. Dabei wird in der Literatur primär zwischen dem dominanten und dem fürsorglichen Kommunikationsstil der Ärzte differenziert (Krupat et al. 2000). Dominanz beschreibt unter anderem Führung, Überlegenheit und Kontrolle. Übertragen auf die Arzt-Patienten-Beziehung ergeben sich daraus verschiedene Möglichkeiten der Dominanz. Ein Mediziner hat die Kontrolle darüber, wie viel der ihm vorliegenden Informationen er preisgibt. Medizinstudenten erfahren in ihrer praktischen Tätigkeit, dass es Situationen im klinischen Alltag gibt, in denen den Patienten nicht die komplette Wahrheit mitgeteilt wird. Ein Beispiel dafür wäre ein schlechtes Diagnoseergebnis bei einer ohnehin infausten Prognose. Besondere Relevanz haben Kontrolle und Dominanz, wenn es um Diagnostik, Medikamentenauswahl und Therapieentscheidungen geht. Mit Hilfe einer dominanten Kommunikationsweise kann der Arzt das Verhalten des Patienten so beeinflussen, wie es aus ärztlicher Sicht richtig erscheint. Dabei spiegelt sich die asymmetrische Beziehung zwischen Arzt und Patient wider. Die 
Gesprächsführung kann dabei eher autoritär als kooperativ sein. Bei der autoritären Form kontrolliert der Arzt das Gespräch, während der Patient nur als Adressat miteinbezogen wird (Müller 2004). Der dominante Kommunikationsstil von Ärzten ist gekennzeichnet durch den Einsatz geschlossener Fragen und die Verwendung medizinischer Fachbegriffe. Dabei wird das medizinische Wissen als Machtinstrument betont. Durch das so demonstrierte Wissen sind Patienten eher dazu geneigt, ihrem Arzt zu vertrauen und Entscheidungen zu überlassen.

Auch die menschliche Stimme trägt zum Rollenverständnis bei. Da eine erhöhte Sprechstimmenlautstärke mit überzeugendem Auftreten (Dominanz) assoziiert ist (Eisler et al. 1973), sollte dieser Aspekt in dieser Arbeit mit betrachtet werden.

Die Psychologin Marianne Schmid Mast und ihre Mitarbeiter führten im Jahr 2006 eine Studie durch, um die Reaktionen von ,Patienten' auf dominanten Kommunikationsstil zu erfassen. Dafür ließ man 167 Studenten als analoge Patienten mit einem animierten, virtuellen Arzt interagieren. Die analogen Patienten wurden dafür in 4 verschiedene Gruppen randomisiert und jeder Gruppe ein anderer virtueller Arzt zugeteilt. Die animierten Ärzte unterschieden sich in dem Grad ihrer dominanten Gesprächsführung während einer virtuellen medizinischen Visite. Die Wahl der computergenerierten Methode ermöglicht hohe experimentelle Kontrolle für die Rahmenbedingungen der Studie. Bei den Gesprächen wurde das verbale und nonverbale Verhalten der analogen Patienten erhoben und nachträglich in einem standardisierten Fragebogen ihr Eindruck vom virtuellen Arzt erfasst. Je dominanter der virtuelle Arzt in der Visite auftrat, desto mehr stimmten die analogen Patienten ihm zu, waren seiner Meinung und desto weniger sprachen sie selbst. Demonstriert ein Arzt also Dominanz, führt das zu Gehorsam und Kooperation seitens der Patienten (Schmid Mast et al. 2008).

Diese Beobachtung ist zum einen interessant im Hinblick auf die asymmetrische Beziehung zwischen Arzt und Patient. Die in diesem Zusammenhang häufig verwendeten Begriffe Autorität und Dominanz besitzen eine negative Wertungskomponente. Oft wird die paternalistische Autorität von Ärzten kritisiert. Das Verhalten von Medizinern ist geprägt durch die Art, wie sie den Menschen sehen. Raz und Fadlon beschreiben einen "Verlust der Persönlichkeit" und eine „anatomische Betrachtung" der Patienten (Raz and Fadlon 2006, S. 56). Auch die Bezeichnung eines Patienten als ein Fall oder medizinisches Projekt wird hier kritisiert (Barrett 1988).

Zum anderen müssen die Ergebnisse der Studie von Schmid Mast unter dem Gesichtspunkt Zeitmangel diskutiert werden. Häufig gibt es zu wenig Personal für zu viele Patienten. Medizinstudenten bemerken, dass das Gelehrte an der Universität häufig nicht kongruent ist mit der klinischen Tätigkeit. Wie man Anamnesen theoretisch führen soll und wie sie meist praktisch umgesetzt werden, ist widersprüchlich. Es bleibt keine Zeit für ausführliche Patientengespräche. Schon Studenten registrieren bei der 
Arbeit im klinischen Alltag, dass manche Patienteninformationen langweilen oder ungeduldig werden lassen (Raz and Fadlon 2003). Dieses Phänomen kann sowohl die Studenten selbst betreffen oder aber auch ihre betreuenden Ärzte bei der Patientenübergabe bzw. -vorstellung. „Sie wollen nicht die Geschichte einer Person hören. Sie wollen die bearbeitete Version hören" (Good 1994, S. 78). Bei dominantem Auftreten sprechen die Patienten weniger und stimmen mehr zu, was Arzt-PatientenGespräche verkürzt (Schmid Mast et al. 2008).

Dominanz bei Ärzten und erhöhte Sprechstimmenlautstärke korrelieren positiv miteinander. In einer Studie von Jinni Harrigan und ihren Mitarbeitern aus dem Jahre 1989 wurden Arzt-Patienten-Telefongespräche analysiert. Die Analysen fokussierten sich auf die paralinguistischen Kommunikationsmittel, sie sollten Rückschlüsse auf die Charaktereigenschaften der Gesprächspartner zulassen. Grundlage der Studie bildeten Telefongespräche von insgesamt 31 Ärzten, die sie mit ihren Patienten geführt hatten. Diese Gespräche wurden 60 Psychologiestudenten/innen vorgestellt, sowohl als Audioversionen als auch als Transkripte. Daraufhin haben die Studenten ihre Wahrnehmungen und Eindrücke in standardisierten Fragebögen festgehalten. Die Ergebnisse der Fragebögen wurden mit verschiedenen statistischen Verfahren ausführlich ausgewertet. Explizit interessant für die Stimmstudie ist das Ergebnis, dass die Ärzte, die laut und schnell sprachen, von den Studenten als dominant beurteilt wurden. Es bestand eine signifikante $(p<0,05)$ und positive Korrelation $(r=0,42)$ zwischen Dominanz und Stimmlautstärke (Harrigan et al. 1989).

Die Studien von Marianne Schmid Mast und Jinni Harrigan bieten Erklärungsansätze für die vorgelegte Arbeit. Forschungen haben ergeben, dass im ärztlichen Alltag ein dominanter Gesprächsstil mit einer lauten Stimme geführt wird. Patienten werten das bei ihren Ärzten als Zeichen von Kompetenz, Autorität und Selbstsicherheit. Sie reagieren darauf mit Zustimmung und stellen weniger Fragen, so dass die Arzt-PatientenGespräche insgesamt kürzer ausfallen. Auf Grundlage der bisherigen Forschungsergebnisse soll die Stimmstudie untersuchen, ob sich Medizinstudenten während des Studiums eine lautere Stimmlautstärke aneignen.

\subsection{Zusammenfassung}

Der Stand der Forschung bietet Anknüpfungspunkte für die hier vorliegende Studie. Viele verschiedene Studien haben sich in der Vergangenheit mit der Arzt-PatientenKommunikation befasst. Dem Gespräch zwischen Arzt und Patient wird eine sehr große Wichtigkeit zugeschrieben. Ob eine Konsultation und sogar die Therapie erfolgreich sind, wird auch durch das Gespräch beeinflusst. Es trägt zum Rollenverständnis bei. Leider beziehen sich die Ergebnisse zumeist auf die gewünschte Gesprächsführung, weniger auf die Stimmführung. 
Zum Thema Stimme gibt es ebenfalls zahlreiche Literatur, gerade im Bereich ihrer Pathologien. Es ist bekannt, dass sich die Stimme im Laufe des Lebens verändert. Beispielsweise nimmt eine alternde Stimme an Stimmlautstärke ab (Ramig et al. 2001). Sozialpsychologisch wird die Stimme im Zusammenhang mit ihrer Wirkung auf die Mitmenschen und als Ausdruck von Persönlichkeit betrachtet. Die Stimmenlautstärke dient als paralinguistisches Kommunikationsmittel. Das Gesprochene wird akustisch durch Lautstärke gestaltet und die Bedeutung moduliert. Die Lautstärke der Stimme vermittelt Charakterzüge und Emotionen des Sprechers.

Die Themen ,professionelle Sozialisation“ und ,professionelle Identität im Medizinstudium bieten diverse Forschungsschwerpunkte. Die Persönlichkeitsentwicklung der Medizinstudenten umfasst einen großen Bereich der medizinischen Soziologie. Es ist schwer, einen Sozialisationsprozess in Form messbarer Werte zu erfassen. Möglicherweise kann er aber zumindest annäherungsweise durch Surrogatparameter (wie zum Beispiel die Stimmlautstärke) abgebildet werden. Die Sprechstimmenlautstärke lässt sich messen und quantifizieren. Lautes Sprechen und Durchsetzungsfähigkeit stehen in engem Zusammenhang miteinander (Eisler et al. 1973) und die Studie der vorgelegten Arbeit nutzt diesen Zusammenhang. Über die Veränderung der Stimmenlautstärke im Medizinstudium gibt es einige wenige Anhaltspunkte, aber keine verlässlichen Daten. 


\section{Fragestellung und Ziel}

Die vorliegende Studie soll die Frage beantworten, ob die Sprechstimme bei Medizinstudenten am Ende des Studiums lauter ist als bei Medizinstudenten im ersten Semester. Sollte sich die Fragestellung bestätigen, ergeben sich Rückschlüsse auf die Entwicklung der Sprechstimmenlautstärke während des Medizinstudiums.

Darüber hinaus wird analysiert, ob sich Mediziner im fortgeschrittenen Studienabschnitt bezüglich ihrer Charaktereigenschaften (wie zum Beispiel Durchsetzungsfähigkeit und Selbstsicherheit) von Medizinern im ersten Semester unterscheiden. Die Ergebnisse können mit professionellen Sozialisationsprozessen im Medizinstudium in Zusammenhang gebracht werden. Das Medizinstudium wird verstanden als ein Prozess von Sozialisation in den Arztberuf (Dornan and Bundy 2004). Es ist charakterisiert durch die Entwicklung einer sogenannten professionellen Identität. Zahlreiche Studien haben sich mit diesem Begriff auseinandergesetzt (u.a. Lingard et al. 2003, Irvine 1999, Pitkala and Mantyranta 2003, Radcliffe and Lester 2003). Als Ergebnisse dieser Studien wurden diverse Ansprüche definiert, die an Medizinstudenten während ihrer Ausbildung gestellt werden; unter anderem müssen Studierende der Medizin lernen, Selbstvertrauen und Zuversicht zu demonstrieren. Unsicherheit macht wenig Eindruck auf Dozenten, Kommilitonen und Patienten. Das Studium trainiert die angehenden Ärzte darin, sich zu behaupten und durchzusetzen. Sie müssen sich der Entwicklung eines professionellen Status und ihrer zukünftigen Arztrolle bewusst werden (Stern and Papadakis 2006).

Kommunikation kann Medizinstudenten helfen, in die Arztrolle hineinzuwachsen und sie zu repräsentieren. Sie unterstreicht die professionelle Persönlichkeit. Die Stimme dient hierfür als Arbeitsinstrument. Anhand der Stimme ziehen Menschen Rückschlüsse auf die Persönlichkeit des Gesprächspartners. Die Stimmgebung ist immer auch ein Stück Selbstdarstellung der sprechenden Person. Mit Hilfe der Stimme kann man Selbstsicherheit und Kompetenz demonstrieren - Attribute, die Ärzten zugeschrieben werden. Es gilt zudem als berufsmotivierend, durch die eigene Stimme den Patienten oder Kollegen zu erreichen und sogar das Verhalten zu beeinflussen.

Die Stimme ist steigerungsfähig durch die Erhöhung ihrer Lautstärke. Wenn man nun davon ausgeht, dass eine Steigerung der Stimmlautstärke auch eine Steigerung der professionellen Persönlichkeit mit sich bringt, dann lässt sich dieser Parameter sehr gut in die Sozialisationsprozesse der Medizinstudenten integrieren.

In der Stimmstudie wird weiterführend der Unterschied zwischen den Stimmlautstärken von Nichtmedizinern im ersten und im fortgeschrittenen Semester untersucht und mit den Ergebnissen der Mediziner verglichen. Es wird die Hypothese geprüft, dass gegen Ende des Studiums die Lautstärke der Stimme bei den Medizinstudenten im Vergleich 
zu Studenten anderer Fachrichtungen lauter ist. Dieser Vergleich hätte dahingehend Implikationen, dass auf dem Weg zum Arztberuf die professionelle Persönlichkeitsentwicklung stärker ausgeprägt ist als bei anderen Akademikern. Die dargelegte Stimmstudie greift demnach 2 verschiedene wissenschaftliche Forschungsschwerpunkte auf: Zum einen die Entwicklung der professionellen Identität im Medizinstudium - auch im Vergleich zu anderen Studiengängen - und zum anderen den Zusammenhang zwischen professioneller Persönlichkeit und Stimmenlautstärke. 


\section{Material und Methoden}

\subsection{Methodische Anlage der Untersuchung}

Bei der vorliegenden Studie handelt es sich um eine Querschnittstudie, die durch entsprechendes soziodemografisches Matching quasi-longitudinale Vergleiche der

Stimmlautstärke zwischen Studierenden des 1. und des 9./10. Fachsemesters ermöglichen soll.

\subsection{Auswahl der Probanden}

Das Studienkollektiv umfasst 2 Kohorten, zum einen Medizinstudenten (Untersuchungspopulation) und zum anderen eine zufällige Auswahl von Studenten anderer Fachrichtungen (Vergleichspopulation). Des Weiteren wird innerhalb dieser beiden Kohorten differenziert zwischen Studierenden des 1. Fachsemesters und des 9./10. Fachsemesters. Die Auswahl der Probanden lässt Aussagen zu über die Entwicklung der Stimmlautstärke innerhalb der beiden Populationen, aber vor allem auch im Vergleich zwischen Medizinstudenten und Nicht-Medizinstudenten.

\subsection{Rekrutierung der Probanden}

Die Rekrutierung erfolgte vorrangig im Universitätsklinikum Göttingen und in den Göttinger Mensen. Während der Mittagszeit wurden hier die Studenten angesprochen. Nach einer kurzen Vorstellung der eigenen Person fragte man sie, in welchem Studiensemester sie sich zu diesem Zeitpunkt befanden. Bei entsprechend passenden Semestern wurde das Projekt kurz beschrieben und ein Handout ausgereicht (Anlage 1). Dabei wurde bewusst nur erklärt, dass Studieninhalt die menschliche Stimme ist, der Parameter Lautstärke wurde zu keiner Zeit erwähnt. Stieß das Projekt auf Interesse, wurde ein Termin vereinbart und Studienfach, Fachsemester und Kontaktdaten (Telefonnummer und/oder E-Mail-Adresse) der Studierenden notiert. Ergänzend zur direkten Ansprache der Studenten wurden Aushänge über die Stimmstudie in zentralen Universitätsgebäuden angebracht (Anlage 2). Bei Interesse konnten die Studenten eine E-Mail an die Online-Stimmstudienadresse (stimmstudie@gmx.de) schreiben und erhielten Studieninformationen, gegebenenfalls wurde ein Termin zum Interview vereinbart. Es wurde den Studenten im Aushang außerdem angeboten, sich direkt telefonisch mit den Projektverantwortlichen Prof. Dr. Wolfgang Himmel, Prof. Dr. Christiane Kiese-Himmel oder Prof. Dr. Martin Scherer in Verbindung zu setzen. Um eine große Menge von Studenten im 1. Semester zu erreichen, wurde das Projekt in diversen Einführungsveranstaltungen verschiedener Fakultäten vorgestellt. Danach teilte man Studieninformationen und Teilnehmerlisten aus. Bei Interesse konnten die Studenten ihre Namen, E-Mail-Adresse und/oder Telefonnummer in die Listen 
eintragen. Telefonisch oder per E-Mail wurden daraufhin Termine zur Studienteilnahme vereinbart.

\subsection{Ablauf der Datenerhebung}

Nach Terminvereinbarung wurden die Studenten zu einem Gespräch in die Abteilung Allgemeinmedizin eingeladen. Jedes Gespräch fand im Konferenzraum 2.102 im 2. Stock statt. Dabei saßen sich Interviewer und Proband an einem Tisch gegenüber. Die genaue Platzierung von Tisch und Stühlen war festgelegt und wurde über den Zeitraum der Messungen am Boden mit Klebeband gekennzeichnet. Die Sitzordnung wählte man so, dass die Position der Stühle und des Tisches während der Aufnahmen nicht verändert werden musste. Vor dem Interviewer stand ein Notebook mit entsprechender Software. Das Programm ,lingWAVES Stimmfeld Plus' war am Laptop bereits aktiviert (Vgl. detaillierter Kap. 4.5.1). Zwischen Interviewer und Proband befand sich der Schallpegelmesser zur Aufnahme der Sprechstimmenlautstärke. Auch die exakte Position des Schallpegelmessers wurde durch Klebeband markiert. Die Fenster im Konferenzraum blieben während der Messungen geschlossen. Um akustische Störungen der Aufnahmen weitestgehend zu vermeiden, war von außen ein Zettel mit der Aufschrift ,BITTE NICHT STÖREN: AUFNAHME!' an die Zimmertür angebracht. Der Studienteilnehmer wurde darauf hingewiesen, sich während der Aufnahmen möglichst ruhig zu verhalten. Unnötige Körperbewegungen, Verrücken des Stuhls und das Stellen von Gegenfragen sollten unterbleiben, um optimale Ergebnisse der Stimmenaufzeichnung zu erreichen.

Den Probanden wurde zunächst die Einverständniserklärung zur Studie vorgelegt (Informed Consent, Anlage 3). Nach der Unterzeichnung konnte die Datenerhebung beginnen.

Die Datenerhebung bestand aus 3 Teilen:

Teil 1: Im ersten Teil der Datenerhebung sollte der Student einen Standardtext vorlesen (,Nordwind und Sonne', Anlage 4). Der Schallpegelmesser maß die Lautstärke der Probandenstimme beim Vorlesen. Das Programm ,lingWAVES‘ wurde vom Interviewer bei Beginn des Textvortrags eingeschaltet und direkt nach dem Vorlesen ausgestellt. Somit war sichergestellt, dass nur die Stimme des Probanden in der Aufnahme erfasst wurde.

Teil 2.: Der zweite Teil der Datenerhebung bestand aus der Schallpegelmessung bei einem halbstandardisierten Interview. Der Interviewführer stellte dem Probanden 3 allgemeine Fragen zum Studium (1. „Warum studierst du Medizin (so formuliert bei Medizinstudenten, bei Studenten anderer Fachrichtungen wurde hier das zutreffende Studium benannt)?", 2. „Was gefällt dir besonders gut an deinem Studium?“, 3. „Was 
gefällt dir nicht an deinem Studium?"). Waren die Antworten des Studenten sehr kurz, konnte gegebenenfalls noch eine beliebige Zusatzfrage gestellt werden. Auch hier wurde das ,lingWAVES'-Programm vom Interviewer nur dann aktiviert, wenn der Proband gesprochen hat. Beim Stellen der Fragen wurde es gestoppt, damit nur die Stimme des Studenten im Stimmfeld erschien.

Teil 3: Im Anschluss an die Tonaufnahmen sollten vom Studienteilnehmer soziodemografische und psychologische Daten erfasst werden. Dafür erhielt er eine standardisierte Auswahl an Fragen aus verschiedenen Fragebögen (Vgl. detaillierter Kap. 4.5.2).

\subsection{Instrumente der Datenerhebung}

\subsection{1 lingWAVES Stimmfeld Plus}

In den ersten beiden Teilen der Datenerhebung wurden die Sprechstimmenlautstärken der Studienteilnehmer gemessen. Die Messungen erfolgten mit Hilfe des SoftwareProgrammes ,lingWAVES Stimmfeld Plus' und dem dazugehörigen Schallpegelmesser. Die Software erlaubt eine genormte und anerkannte Stimmfeldmessung. Sie wird auch an logopädischen Schulen und HNO-/ Phoniatrie-Bildungseinrichtungen verwendet. Bei der Aufnahmetechnik mit der ,lingWAVES'-Software werden Schallpegel und Tonhöhe der Stimme zeitgleich zum Sprechen gemessen. Die Darstellung der Messergebnisse erfolgt in einem sogenannten Stimmfeld beziehungsweise Phonetogramm (Anlage 5). Daraus lässt sich der Mittelwert der Stimmlautstärke ermitteln. Für beide Messsituationen (Vorlesen und halbstandardisiertes Interview) wurden die Mittelwerte bestimmt und später bei der Datenanalyse verwendet.

\subsubsection{Fragebögen}

Die ausgewählten Fragebögen erfassten soziodemografische und psychologische Daten der Studienteilnehmer. Fünf verschiedene Fragebögen bildeten die Grundlage der standardisierten Fragenauswahl:

(1) lingWAVES Fragebogen VDI-Stimmstörungsindex (年lage 6)

(2) Soziodemografische Fragen (Anlage 7)

(3) 7 ausgewählte Fragen aus dem IAF (Anlage 8)

(4) 12 Fragen aus dem FPI-R (

(5) HADS-D ( 


\subsubsection{1 lingWAVES Fragebogen VDI-Stimmstörungsindex}

„Der Stimmstörungsindex (SSI), englisch Voice Disorder Index (VDI), ist in Europa ein eingeführter und evaluierter Bewertungsindex für die Einschätzung der stimmbezogenen Lebensqualität eines Patienten" (lingWAVES Handbuch 2005, S. 35). Nawka et al. entwickelten den SSI durch eine Auswahl von insgesamt 12 Items. Grundlage dieser Items bilden negative Stimmerfahrung, Selbstunsicherheit, mangelnde Tragfähigkeit der Stimme und negative Emotionalität (Nawka et al. 2003). Die Beantwortung pro Item wird kategorisiert von 0 (nie) bis hin zu 4 (immer). Somit ergibt sich für jeden Probanden aus diesen 12 Items ein errechneter Score zwischen 0 und 48. Daraus lassen sich mit Hilfe von Grenzwerten 4 verschiedene Ausprägungen von stimmlichen Handicaps ermitteln.

\subsubsection{Soziodemografische Fragen}

In der Anlage 7 findet sich eine Auswahl soziodemografischer Fragen. Die Erhebung von Studienfach und Semesterzahl der Probanden bildet die Voraussetzung für die 4 verschiedenen Fallgruppen der Studie. Die Angabe von Lebensalter und Geschlecht der Studenten ist notwendig, um die Stichprobe grundsätzlich zu beschreiben und die Verteilung innerhalb der 4 Fallgruppen darzulegen. Die soziodemografischen Fragen evaluieren außerdem individuelle Gegebenheiten der Probanden, die Einfluss auf die Stimmentwicklung haben könnten (derzeit bzw. jemals logopädische Therapie, Sprachoder Stimmtherapie, Schwerhörige im nahen Umfeld, Sporttrainer oder Lehrender in Freizeit, etc.). Die Probanden sollten in diesem Fragebogen ihre eigene Stimmstärke beim Sprechen selbst einschätzen. Aus dieser Angabe wurde untersucht, ob die tatsächlich berechnete Stimmstärke mit der Selbsteinschätzung übereinstimmt.

\subsubsection{Der Interaktions-Angst-Fragebogen}

Der Interaktions-Angst-Fragebogen (IAF) ist ein psychologischer Fragebogen, der Angstneigungen in verschiedenen Situationen erfasst. Für die vorgelegte Studie wählte man die Skala ,Angst vor Selbstbehauptung' (=IAF Skala 5) aus, welche aus insgesamt 7 Items besteht. Jedem Item wird ein Rating von 1 (ziemlich angenehm) bis 7 (äußerst unangenehm) zugeordnet. Somit ergibt sich für jeden Probanden aus diesen 7 Items ein errechneter Rohwert zwischen 7 und 49. Mit Hilfe des IAF-Handbuches und darin aufgelisteter geschlechtsspezifischer Normtabellen werden aus den errechneten Rohwerten standardisierte T-Werte bestimmt. Diesen T-Werten liegt eine umgewandelte Prozentrangskala zugrunde. Daraus ergeben sich T-Werte zwischen 0 und 100 mit einem Mittelwert von 50 und einer Standardabweichung von 10. „Ein hoher Testwert kann hinweisen auf: niedriges Selbstkonzept eigener Fähigkeiten, geringe Selbstwirksamkeitsüberzeugung; geringe Selbstsicherheit; geringe internale Kontrollüberzeugung. Ein niedriger Testwert kann hinweisen auf: ein hohes 
Selbstkonzept eigener Fähigkeiten; hohe Selbstwirksamkeitsüberzeugung; Selbstsicherheit; Selbstbehauptung und Dominanz; internale Kontrollüberzeugung und hohe Selbstachtung“" (Becker 1997, S. 17).

\subsubsection{Das Freiburger Persönlichkeitsinventar}

Das Freiburger Persönlichkeitsinventar (FPI-R) dient zur Erfassung von verschiedenen Persönlichkeitsstrukturen. In dem kompletten Fragebogen werden insgesamt 12 psychologische Attribute bestimmt. In der Stimmstudie fand die Subskala ,Aggressivität Verwendung. Diese Skala setzt sich aus 12 Items zusammen, deren Aussagen mit ,stimmt' und ,stimmt nicht' bewertet werden können. Aus der Häufigkeit der mit ,stimmt beantworteten Items wird ein Rohwert berechnet. Demzufolge lassen sich Rohwerte zwischen 0 und 12 ermitteln. Die Rohwerte weisen geschlechts - und altersabhängige Unterschiede auf. Um die Probanden besser vergleichen zu können, wurden ihre Rohwerte in standardisierte Werte transformiert. Aus Normtabellen wurde jedem Rohwert ein zugehöriger Stanine-Wert zwischen 1 und 9 zugeordnet, für die StanineWerte liegt der Mittelwert bei 5 und die Standardabweichung bei 2 (Fahrenberg et al. 2001). Niedrige Testwerte beschreiben einen Menschen als „zurückhaltend“ und „kontrolliert", hohe Testwerte dagegen als „sich durchsetzend“, „spontan und reaktiv“ (Fahrenberg et al. 2001, S. 75).

\subsubsection{Die Hospital Anxiety and Depression Scale}

„Die Hospital Anxiety and Depression Scale (HADS) ist ein kurzer Selbstbeurteilungsfragebogen zur Erfassung von Angst und Depressivität“" (HerrmannLingen et al. 2005, S. 4). In der vorliegenden Studie wurde die deutsche Version der HADS verwendet, für diese Version steht allgemeingültig die Abkürzung HADS-D. Der Fragebogen besteht aus insgesamt 14 Items, daraus bildet man 2 Subskalen - die Depressivitätsskala (=HADS-D/D) und die Angstskala (=HADS-D/A). Jeder Item wird 4stufig kategorisiert, pro Item erreicht man je nach Antwort 0 bis 3 Punkte. Durch Addition ergibt sich daraus für jede der beiden Subskalen ein Gesamtscore zwischen 0 und 21. Probanden mit einem HADS-D/D-Wert $>8$ werden als depressiv beschrieben, Probanden mit einem HADS-D/A-Wert > 10 gelten als ängstlich (Herrmann-Lingen et al. 2005, Scherer M et al. 2007).

\subsection{Datenanalyse}

Zusätzlich zu einfachen Häufigkeitsaufzählungen wurde mit Hilfe von $x^{2}$-Tests und $t$ Tests für unabhängige Stichproben (zweiseitig getestet bei $\alpha=0,05$ ) überprüft, ob die vier Fallgruppen soziodemografisch vergleichbar sind. Eine Clusteranalyse klassifizierte 
die gesamte Studienpopulation nach dem Kriterium ,Stimmlautstärke‘ in 3 verschiedene Kategorien.

Das Zielkriterium der Messungen war die Sprechstimmenlautstärke. Aus den beiden Messsituationen ,Vorlesen' und ,halbstandardisiertes Interview" wurden jeweils die Mittelwerte der Sprechstimmenlautstärke bestimmt. Durch eine zweifaktorielle Varianzanalyse (ANOVA) ließ sich der Einfluss des Studienfaches und der Zeit beurteilen. Auch die Interaktion ,Studienfach $x$ Zeit' wurde erfasst. Die für Varianzanalysen notwendige Normalverteilung der abhängigen Variablen konnte mit der Datendarstellung der Stimmlautstärke als Boxplots veranschaulicht werden. Mit Hilfe von Regressionsanalysen wurde weiterhin nach Einflussfaktoren auf die Stimmlautstärke gesucht.

Zur Datenanalyse wurde das Statistikprogramm SPSS (Statistical Package for the Social Sciences) für Windows (Version 18 und 19) verwendet. Das festgelegte Signifikanzniveau lag bei $5 \%$.

\subsection{Datenschutz}

Die Richtlinien zum Datenschutz wurden in dieser Studie streng eingehalten. Ein Antrag der Studie wurde der Ethik-Kommission vorgelegt und genehmig (Kennzeichen des Ethikvotums: 8/1/09). Alle Mitarbeiter und Mitarbeiterinnen der Studie unterschrieben eine Verschwiegenheitserklärung. Zusätzlich dazu wiesen die Studienleiter alle Mitarbeiter mündlich in den strengen Umgang mit den Daten ein. Die Datenbank wurde für Außenstehende durch ein Passwort geschützt. Jeder Studienteilnehmer erhielt eine Kennnummer. Die Stimmfelder und ausgefüllten Fragebögen waren nur mit der Kennnummer beschriftet. Die Einverständniserklärungen mit den Namen der Teilnehmer wurden separat von den persönlichen Daten in einem verschlossenen Stahlschrank in der Abteilung Allgemeinmedizin aufbewahrt (Pseudonymisierung). Nur die Studienverantwortlichen und direkte Mitarbeiter der Studie konnten auf die Namen zugreifen. Eine Verknüpfung de pseudonymisierten Studiendaten und der zugehörigen Person war nur den Leitern und Mitarbeitern der Studie möglich und hätte hohen Aufwand erfordert. Nach der Stimmenaufnahme wurde der Text selbst direkt gelöscht. Gespeichert wurden nur die Stimmfelder einschließlich der Lautstärke der Stimmprobe.

Jeder Studienteilnehmer erhielt die Studieninformationen und eine Einverständniserklärung. In beiden Formularen war schriftlich festgehalten, dass die erhobenen Daten ausschließlich für die Stimmstudie verwendet werden. Erst nach Unterzeichnung der Einverständniserklärung fanden Messungen statt. Hätte ein Studienteilnehmer die Teilnahme widerrufen, wären der Name und die zugehörigen Daten umgehend gelöscht worden. 


\section{Ergebnisse}

\subsection{Teilnahme}

Die dargelegte Studie setzt sich aus vier verschiedenen Fallgruppen zusammen. Eine Mindestzahl von 50 Probanden pro Fallgruppe wurde angestrebt und erreicht. Stimmstörungen, Erkältungen und/oder Heiserkeit galten als Ausschlusskriterien für die Studienteilnahme. Nach der gesamten Rekrutierung hatten sich insgesamt 250 Studenten in Teilnehmerlisten eingetragen, daraufhin wurde je nach Angaben der Interessenten telefonisch oder per E-Mail Kontakt aufgenommen. 42 Studenten entschieden sich letztendlich doch gegen die Studienteilnahme, sie meldeten sich nicht zurück oder gaben mangelndes Interesse oder fehlende Zeit als Gründe für die Absage an. Von den restlichen 208 Teilnehmern mussten nach abgeschlossener Datenerhebung 2 Probanden von der Studie ausgeschlossen werden, da die Semesterzahl nicht den gesuchten Anforderungen entsprach. Somit verbleiben 206 Teilnehmer, deren Daten die Grundlage dieser Arbeit bilden.

\subsection{Beschreibung der Stichprobe}

\subsection{1 Überblick Fallgruppen}

Die Verteilung der insgesamt 206 Teilnehmer innerhalb der 4 Fallgruppen sowie das Geschlechter- und Altersverhältnis kann aus Tabelle 1 entnommen werden. In jeder Fallgruppe nahmen mehr Frauen an der Studie teil als Männer, jedoch liegt das prozentuale Geschlechterverhältnis maximal bei 60/40 (Nichtmediziner, $\geq 9$. Semester). Die Erstsemesterstudenten waren bei Studienteilnahme zwischen 20 und 21 Jahren alt, die Studierenden der höheren Semester zwischen 25 und 26. Keiner der Studenten nahm zum Zeitpunkt der Studie eine logopädische Therapie in Anspruch, ein Proband gab an, aktuell an einem Stimmtraining teilzunehmen. Im Rahmen des soziodemografischen Fragebogens wurde unter anderem erhoben, ob sich im Umfeld der Probanden Schwerhörige befinden. Je nach Fallgruppe wurde diese Frage von 21 bis $32 \%$ der Probanden mit ,ja' beantwortet. 
Tabelle 1: Überblick Stichprobe

$\begin{array}{llll}\text { Med., } & \text { Med., } & \text { sonst. Fach, } & \text { sonst. Fach, } \\ \text { 1. Sem. } & \geq 9 . \text { Sem. } & 1 . \text { Sem. } & \geq 9 . \text { Sem. }\end{array}$

\begin{tabular}{|c|c|c|c|c|}
\hline - Anzahl (\%)* & $51(49)$ & $53(51)$ & $50(49)$ & $52(51)$ \\
\hline - $\quad$, $\mathrm{n}(\%)^{* *}$ & $28(55)$ & $29(55)$ & $29(58)$ & $31(60)$ \\
\hline - $\quad \hat{\sigma}, \mathrm{n}(\%)^{* *}$ & $23(45)$ & $24(45)$ & $21(42)$ & $21(40)$ \\
\hline - Alter (SD) & $20,9(2,0)$ & $25,6(1,8)$ & $20,4(1,4)$ & $25,7(1,7)$ \\
\hline
\end{tabular}

Die Vergleichsgruppe der Nichtmediziner $(\mathrm{N}=102)$ setzte sich aus einem breiten Spektrum verschiedener Studienrichtungen zusammen. Es wurde bei der Rekrutierung großer Wert darauf gelegt, dass keine spezielle Fachrichtung überrepräsentiert war. Einen Überblick über die heterogene Verteilung der Studienfächer erlaubt das Diagramm in Abbildung 1. Dabei ist zu beachten, dass innerhalb der verschieden Fachrichtungen ebenfalls eine hohe Vielfalt an diversen Fächerkombinationen vorlag. Bei der Einteilung wurde jeweils das angegebene Hauptfach der Studenten berücksichtigt. Im Kreisdiagramm unter ,Sonstige' aufgelistet wurden all die Studiengänge, die in dieser Untersuchung nur einmalig vertreten waren (insgesamt 8 verschiedene Studiengänge). 


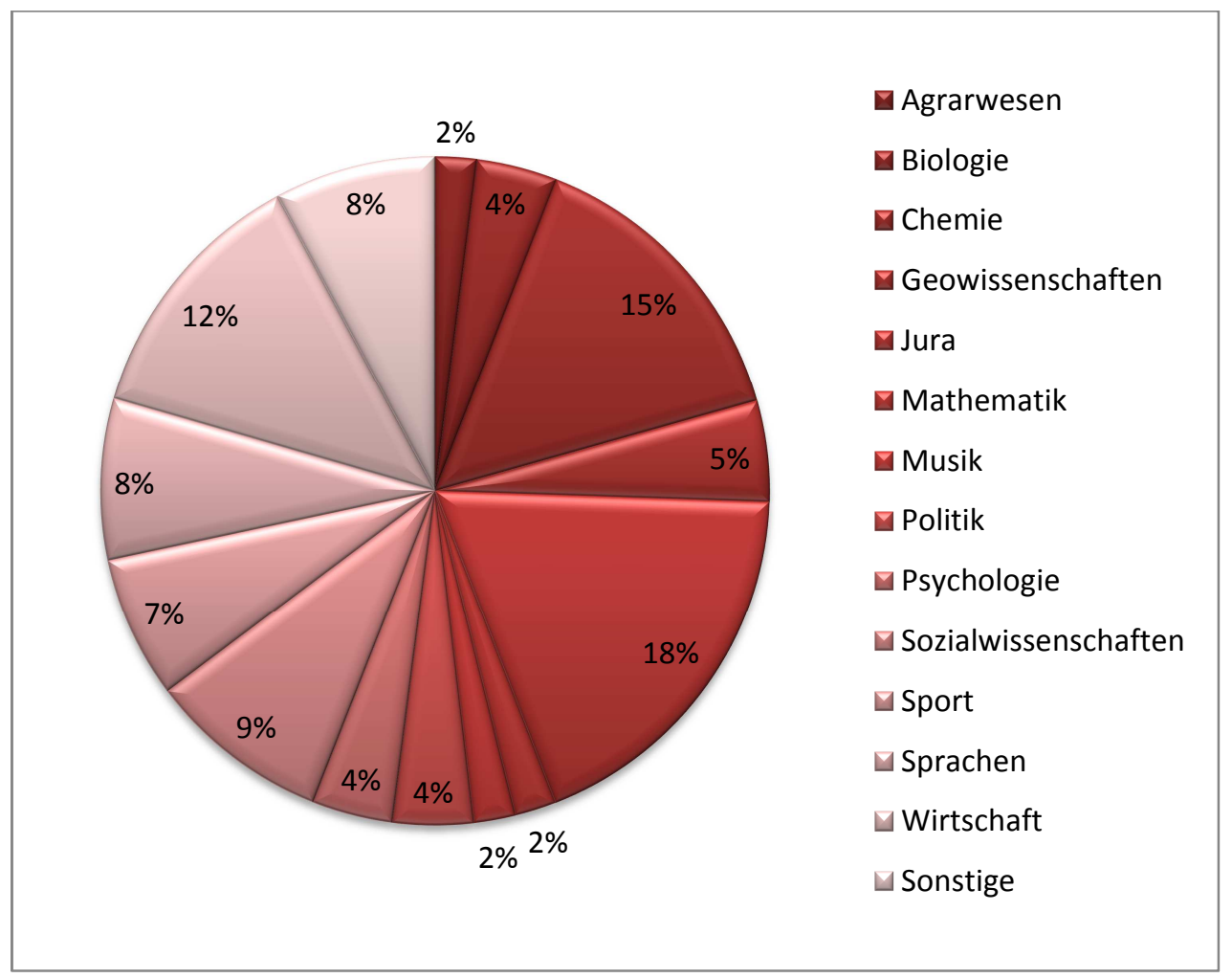

Abbildung 1: Studienrichtungen der Vergleichsgruppe

\subsubsection{Vergleich der Fallgruppen bezüglich soziodemografischer Daten}

Bei der vorgelegten Studie handelt es sich um eine Querschnittsstudie. Für quasilongitudinale Vergleiche war ein soziodemografisches Matching erforderlich. Der Fokus lag dabei primär auf den beiden Eigenschaften Alter und Geschlecht. Die Fallgruppen mussten in Alters- und Geschlechterverteilung vergleichbar sein, so dass Alters- und Geschlechterunterschiede keine Rückschlüsse auf die durchschnittlichen Stimmlautstärken zuließen (Vgl. detaillierter Kap. 2.1). Alle 4 Fallgruppen wurden bezüglich ihrer Geschlechterrelationen verglichen. Der Chi-Quadrat-Test berechnete einen $p$-Wert von 0,947. Somit gab es keine signifikanten Geschlechterunterschiede zwischen den verschiedenen Untersuchungsgruppen. Differenzen der Stimmlautstärken - sowohl zwischen den jüngeren und den älteren Semestern der gleichen Studiengruppen als auch zwischen den Medizinern und Nichtmedizinern der gleichen Semesterzahl - ließen sich also nicht zurückführen auf eine ungleiche Geschlechterverteilung.

Weiterhin durften zwischen den beiden Gruppen der Erstsemesterstudenten (Mediziner versus Nichtmediziner) und den beiden Gruppen der höheren Semester (Mediziner versus Nichtmediziner) keine signifikanten Altersunterschiede vorliegen, die einen Unterschied der Stimmlautstärke erklärt hätten. Der t-Test ergab bei der Untersuchung der Altersverteilung zwischen den Erstsemesterstudenten einen p-Wert von 0,172 und 
bei der Untersuchung der Studenten in den höheren Semestern einen p-Wert von 0,882. Der Altersunterschied war demnach in beiden Fällen nicht signifikant. Eine Analyse der Altersverteilung zwischen den Gruppen der Mediziner (Erstsemesterstudenten versus Studenten der höheren Semester) und den Gruppen der Nichtmediziner (Erstsemesterstudenten versus Studenten der höheren Semester) wurde nicht durchgeführt, da sich der Altersdurchschnitt nach 5 Jahren Studium in jedem Fall ändert. Die erläuterten Untersuchungen ergaben passende Vergleichsgruppen bezüglich Alter und Geschlecht, das soziodemografische Matching war erfolgt.

Tabelle 2: Ergebnisse $\mathrm{X}^{2}$ - Test

Med., Med., sonst. Fach, sonst. Fach, Total p-Wert

1. Sem. $\geq 9$. Sem. 1. Sem. $\geq 9$. Sem. $\left(x^{2}\right)$

Geschlecht

$\begin{array}{lllllll}-\stackrel{+}{+} & 28 & 29 & 29 & 31 & 117 & \\ \text { - } & 23 & 24 & 21 & 21 & 89 & \end{array}$

Tabelle 3: Ergebnisse t-Tests

\begin{tabular}{lllll}
\hline & $\begin{array}{l}\text { Med., } \\
\text { 1.Sem. }\end{array}$ & $\begin{array}{l}\text { sonst. Fach, } \\
\text { 1. Sem. }\end{array}$ & $\begin{array}{l}\text { Med., } \\
\mathbf{2 9 . S e m . ~}\end{array}$ & $\begin{array}{l}\text { sonst. Fach, } \\
\mathbf{9} \text {. Sem. }\end{array}$ \\
\hline $\begin{array}{l}\text { Lebensalter } \\
\text { (SD) }\end{array}$ & $20,9(2,0)$ & $20,4(1,4)$ & $25,6(1,8)$ & $25,7(1,7)$ \\
- p-Wert & 0,172 & 0,882
\end{tabular}

\subsubsection{Einteilung des Studienkollektivs nach Stimmlautstärke}

Im Rahmen der Literaturrecherche war es der Verfasserin nicht möglich, eine allgemein gültige Einteilung der Sprechstimme nach ihrer Lautstärke zu finden. Es schien jedoch interessant für den Hintergrund der Studie, eine Vorstellung davon zu haben, wann eine Stimme als eher leise, normal und eher laut gilt. Für jeden Probanden/jede Probandin wurde aus dem 1. Teil der Stimmfeldmessung (Vorlesen) und dem 2. Teil der 
Stimmfeldmessung (Interview) ein durchschnittlicher Stimmlautstärkewert ermittelt. Mit diesen Werten als Grundlage ließ sich für die Stichprobe der Arbeit eine Clusteranalyse durchführen, welche die Probanden je nach Stimmlautstärkemittelwert in 3 Gruppen (Cluster) einteilte. In diesen Clustern wurden jeweils die Probanden zusammengefasst, die ähnliche Stimmintensitätsmittelwerte bzw. Stimmlautstärken aufwiesen. Ein Mittelwert für die Stimmlautstärke innerhalb der jeweiligen Gruppe sowie die Anzahl der Probanden pro Cluster geht aus Tabelle 4 hervor. Wie zu erwarten, beinhaltet das Cluster der normal lauten Sprecher die meisten Probanden $(\mathrm{N}=108)$. Des Weiteren gab es innerhalb der vorliegenden Stichprobe mehr leise als laute Redner.

Tabelle 4: Ergebnisse Clusteranalyse

\begin{tabular}{|c|c|}
\hline $\begin{array}{l}\text { Cluster } 1 \\
=\text { eher leise }\end{array}$ & $\begin{array}{l}\text { Cluster } 2 \\
=\text { normal laut }\end{array}$ \\
\hline
\end{tabular}

- Lautstärkemittelwert 61,5

65,2

70,2

Sprechstimme in $\mathrm{dB}$

- Anzahl Probanden

(\%)

$63(31)$

$106(51)$

$37(18)$

\subsection{Ergebnisse der Fragebögen}

Einen Überblick über die erhobenen Ergebnisse der Fragebögen liefert Tabelle 5. 
Tabelle 5: Ergebnisse Fragebögen

\begin{tabular}{|c|c|c|c|c|c|c|c|}
\hline Kriterien & Total & $\begin{array}{l}\text { Med., } \\
\text { 1. Sem. }\end{array}$ & $\begin{array}{l}\text { Med., } \\
\geq 9 \text {. Sem. }\end{array}$ & $\begin{array}{l}\text { sonst. Fach, } \\
\text { 1. Sem. }\end{array}$ & $\begin{array}{l}\text { sonst. Fach, } \\
\geq 9 \text {. Sem. }\end{array}$ & $\begin{array}{l}p \\
x^{2}\end{array}$ & $\begin{array}{l}\text { Mittelwert } \\
\text { dB }\end{array}$ \\
\hline $\begin{array}{l}\text { SSI } \\
-\quad \text { kein Handicap (0-7) } \\
-\quad \text { leichtes Handicap (8-14) } \\
\text { - } \quad \text { hochgradiges Handicap (15-22) } \\
\text { handicap (23-48) }\end{array}$ & $\begin{array}{l}136(66 \%) \\
56(27 \%) \\
14(7 \%) \\
\quad-\end{array}$ & $\begin{array}{l}30(59 \%) \\
14(27 \%) \\
7(14 \%) \\
\quad-\end{array}$ & $\begin{array}{l}41(77 \%) \\
11(21 \%) \\
1(2 \%) \\
\quad-\end{array}$ & $\begin{array}{l}32(64 \%) \\
15(30 \%) \\
3(6 \%) \\
-\end{array}$ & $\begin{array}{l}33(63 \%) \\
16(31 \%) \\
3(6 \%) \\
\quad-\end{array}$ & $\begin{array}{c}0,215 \\
-\end{array}$ & $\begin{array}{l}65,3 \\
64,9 \\
62,5\end{array}$ \\
\hline $\begin{array}{l}\text { Selbsteinschätzung } \\
\text { - } \quad \text { eher leise } \\
\text { - } \quad \text { normal } \\
\text { - } \quad \text { eher laut }\end{array}$ & $\begin{array}{l}21(10 \%) \\
132(64 \%) \\
53(26 \%)\end{array}$ & $\begin{array}{l}3(6 \%) \\
36(71 \%) \\
12(23 \%)\end{array}$ & $\begin{array}{l}3(6 \%) \\
33(62 \%) \\
17(32 \%)\end{array}$ & $\begin{array}{l}7(14 \%) \\
29(58 \%) \\
14(28 \%)\end{array}$ & $\begin{array}{l}8(15 \%) \\
34(66 \%) \\
10(19 \%)\end{array}$ & 0,356 & $\begin{array}{l}61,9 \\
64,7 \\
66,9\end{array}$ \\
\hline $\begin{array}{ll}\text { FPI-R } & \\
\bullet & \text { Stanine-Mittelwert } \\
- & \text { zurückhaltend }(\text { Stanine }=1) \\
\text { sich durchsetzend }(\text { Stanine }=9)\end{array}$ & $\begin{array}{l}4,41 \\
4(2 \%)^{*} \\
2(1 \%)^{*}\end{array}$ & $\begin{array}{c}4,25 \\
- \\
-\end{array}$ & $\begin{array}{l}4,60 \\
1(2 \%)^{* \star} \\
-\end{array}$ & $\begin{array}{l}4,42 \\
- \\
-\end{array}$ & $\begin{array}{l}4,37 \\
3(6 \%)^{* *} \\
2(4 \%)^{* *}\end{array}$ & 0,200 & $\begin{array}{l}65,3 \\
68,2\end{array}$ \\
\hline $\begin{array}{ll}\text { IAF } & \\
\cdot & \text { T-Wert Mittelwert } \\
& \text { geringe Selbstsicherheit } \\
& \text { (T-Wert>65) } \\
& \text { hohe Selbstsicherheit } \\
& (\text { T-Wert<35) }\end{array}$ & $\begin{array}{l}51,3 \\
17(8 \%)^{*} \\
12(6 \%)^{*}\end{array}$ & $\begin{array}{l}52,9 \\
10(20 \%)^{* *} \\
5(10 \%)^{* *}\end{array}$ & $\begin{array}{l}50,9 \\
2(4 \%)^{\star *} \\
4(8 \%)^{* *}\end{array}$ & $\begin{array}{l}51,4 \\
2(4 \%)^{\star *} \\
1(2 \%)^{\star *}\end{array}$ & $\begin{array}{l}50,1 \\
3(6 \%)^{\star *} \\
2(4 \%)^{\star *}\end{array}$ & 0,014 & $\begin{array}{l}63,1 \\
66,1\end{array}$ \\
\hline $\begin{array}{l}\text { HADS-D } \\
\qquad \quad \text { Depression }(H A D S-D / D>8) \\
\cdot \quad \text { Angst }(H A D S-D / A>10)\end{array}$ & $\begin{array}{l}8(4 \%)^{*} \\
21(10 \%)^{*}\end{array}$ & $\begin{array}{l}2(4 \%)^{\star *} \\
7(14 \%)^{* *}\end{array}$ & $\begin{array}{l}1(2 \%)^{* *} \\
4(8 \%)^{* *}\end{array}$ & $\begin{array}{l}3(6 \%)^{* *} \\
2(4 \%)^{* *}\end{array}$ & $\begin{array}{l}2(4 \%)^{* *} \\
8(15 \%)^{* *}\end{array}$ & $\begin{array}{l}0,761 \\
0,193\end{array}$ & $\begin{array}{l}64,5 \\
64,8\end{array}$ \\
\hline Mittelwert dB & 65,0 & 64,4 & 66,3 & 65,2 & 64,0 & & \\
\hline
\end{tabular}


Die Ergebnisse des SSI zeigen, dass die Schweregrade der stimmliche Handicaps in allen 4 Fallgruppen nicht signifikant voneinander abwichen $(x 2-T e s t: p=0,215)$. Je schwerer das stimmliche Handicap ermittelt wurde, desto niedriger waren die zugehörigen, durchschnittlichen Stimmlautstärkewerte. Jeder Proband/jede Probandin hat die Lautstärke der eigenen Stimme selbst eingeschätzt und die Ergebnisse der Selbsteinschätzungen wurden mit den tatsächlich errechneten Lautstärkemittelwerten verglichen. Die Gruppe der Studenten, die ihre Stimme als ,eher leise' einstufte, hat einen Lautstärkemittelwert der Sprechstimme von 61,9dB. Demgegenüber wurde für die Probanden mit der Selbsteinschätzung der eigenen Stimme als ,eher laut' ein mittlerer Stimmintensitätswert von $66,9 \mathrm{~dB}$ ermittelt.

Die Ergebnisse des FPI sind in Tabelle 5 - neben der Dichotomisierung - als StanineMittelwerte für jede der 4 Untersuchungsgruppen dargelegt. Wie schon im vorherigen Kapitel dieser Arbeit erläutert, gelten Probanden mit niedrigen Testwerten als eher zurückhaltend und Probanden mit hohen Testwerten als sich durchsetzend. Der Stanine-Mittelwert für die Nichtmediziner im ersten Semester lag bei 4,42 und fiel bei den Nichtmedizinen im fortgeschrittenen Studium auf einen durchschnittlichen Wert von 4,37. Bei den Medizinern war die Entwicklung deutlich gegensätzlich. Hier lag der durchschnittliche Stanine-Mittelwert in der Erstsemestergruppe bei 4,25 (entspricht dem niedrigsten FPI-Mittelwert der 4 Untersuchungsgruppen) und stieg bei den Studierenden der höheren Semester auf einen durchschnittlichen Wert von 4,60 (entspricht dem höchsten FPI-Mittelwert der 4 Untersuchungsgruppen). Die Ergebnisse der FPIAggressionsskala wurden zusätzlich dichotomisiert. Die klassische Festlegung der CutOff-Werte (bestimmt durch Mittelwert +/- 1 Standardabweichung, hier: $M W=5, S D=2$ ) wurde erweitert, da der daraus resultierende auffällige Bereich als zu groß und undifferenziert (31,73\% einer Normstichprobe) erachtet wurde. In dieser Arbeit lagen die Cut-Off-Werte bei Stanine $<2$ und Stanine $>8$. Die gewählten Grenzwerte erklärten den Bereich anderthalb Standardabweichungen um den Mittelwert als sogenannten Normbereich (MW +/- 1,5 SD $=5+/-3=2$ bis 8 ). Probanden, die nicht in diesem Bereich lagen, also mit Stanine-Werten von 1 oder 9, galten als auffällig. Die Gruppe der extrem zurückhaltenden Probanden sprach im Durchschnitt leiser als das Probandenkollektiv, welches sich gerne durchsetzte.

Aus den IAF-Daten wurden ebenfalls Mittelwerte pro Fallgruppe und dichotomisierte Bereiche ermittelt. Die Ergebnisse der Tabelle beziehen sich hierbei auf die zuvor bestimmten T-Werte. Die Dichotomisierung erfolgte analog zum FPI und zeigte auf, dass Probanden mit T-Werten $>65$ eine auffällig geringe Selbstsicherheit und Probanden mit T-Werten $<35$ eine auffällig hohe Selbstsicherheit kennzeichnet. Für die beiden auffälligen Probandengruppen wurde jeweils ein Mittelwert der Stimmlautstärke berechnet. Die selbstsichere Probandengruppe verzeichnete höhere 
Stimmlautstärkewerte als die weniger selbstsichere Probandengruppe $(66,1 \mathrm{~dB}$ gegenüber $63,1 \mathrm{~dB})$.

In dem gesamten Studienkollektiv erfasste die Hospital Anxiety and Depression Scale etwa $10 \%$ ängstliche und $4 \%$ depressive Teilnehmer. Die Prävalenz von Depression und Angst lag bei Medizinern im fortgeschrittenen Semester niedriger als bei den Medizinstudenten im ersten Semester. Bei den Nichtmedizinern gab es bei den Studenten im höheren Semester weniger Probanden mit Depressionen und mehr Probanden mit Angst als in der Gruppe der Erstsemesterstudenten.

\subsection{Ergebnisse der Stimmfeldmessungen}

Bei der Analyse der Stimmfeldmessungen wurden zum einen die beiden stimmlichen Untersuchungen (Teil 1: Vorlesen des Standardtextes ,Nordwind und Sonne', Teil 2: halbstandardisiertes Interview) separat betrachtet und zum anderen aus dem arithmetischen Mittel beider Untersuchungen Erkenntnisse gewonnen (siehe Tabelle 6). Grundsätzlich ließ sich feststellen, dass alle 4 Fallgruppen beim Vorlesen lauter sprachen als im Interview. Die durchschnittliche Lautstärkedifferenz der beiden Untersuchungen lag bei etwa 3,6dB.

Die Medizinstudenten im 1. Semester lasen den Standardtext mit einer Lautstärke von durchschnittlich 66,1dB vor, für die Mediziner im höheren Semester lag beim Vorlesen der Stimmlautstärke-Mittelwert bei $67,8 \mathrm{~dB}$. Daraus ergab sich eine Zunahme der Stimmintensität um $1,7 \mathrm{~dB}$. Die gleiche Entwicklung ließ sich aus den Berechnungen des Interviews schlussfolgern. Während die Erstsemesterstudenten der Medizinergruppe ein Interview bei einem Lautstärkemittelwert von 62,6dB führten, steigerten sich die älteren Mediziner bei der gleichen Untersuchung auf einen Stimmintensitätsmittelwert von $64,7 \mathrm{~dB}$. Bei einer daraus resultierenden Differenz von 2,1dB lag die Zunahme der durchschnittlichen Stimmlautstärke im Interview noch höher als beim Vorlesen. Beide Stimmfeldmessungen in Betracht ziehend, ließ sich für die Mediziner eine Steigerung der Stimmlautstärke von $64,4 \mathrm{~dB}$ auf $66,3 \mathrm{~dB}$ und damit um $1,9 \mathrm{~dB}$ erkennen.

Betrachtete man bei den Nichtmedizinern sowohl den 1. Teil als auch den 2. Teil der Datenerhebung und verglich die Lautstärke-Mittelwerte der Erstsemesterstudenten mit denen der Studenten im höheren Semester, so ließ sich in beiden Untersuchungen eine Abnahme der Stimmlautstärke eruieren. Zu Beginn des Studiums wurde der Standardtext von den Nichtmedizinern mit einer durchschnittlichen Stimmlautstärke von $67 \mathrm{~dB}$ vorgelesen. Für die Nichtmediziner im fortgeschrittenen Studium lag beim Vorlesen der durchschnittliche Lautstärkewert bei $66,1 \mathrm{~dB}$. Daraus ergibt sich eine Abnahme der Stimmlautstärke um 0,9dB. In unserer Studie führten die Nichtmediziner im ersten Semester ein Interview bei einer durchschnittlichen Lautstärke von 63,5dB. 
Für die Gruppe der Nichtmediziner im höheren Semester wurde im Interview ein durchschnittlicher Stimmlautstärkewert von $61,9 \mathrm{~dB}$ erhoben. Somit war die Sprechstimme der Nichtmediziner im höheren Semester in einem Interview im Durchschnitt 1,6dB leiser als die Sprechstimme der Nichtmediziner im ersten Semester. Im Vergleich der berechneten Mittelwerte aus beiden Untersuchungen konnte zwischen den beiden Gruppen der Nichtmediziner eine Abnahme der Stimmintensität von $65,2 \mathrm{~dB}$ auf $64 \mathrm{~dB}$ und damit um $1,2 \mathrm{~dB}$ dokumentiert werden.

Tabelle 6: Mittelwerte Stimmfeldmessungen

$\begin{array}{llll}\text { Med., } & \text { Med., } & \text { sonst. Fach, } & \text { sonst. Fach, } \\ \text { 1. Sem. } & \geq 9 . \text { Sem. } & \text { 1. Sem. } & \geq 9 . \text { Sem. }\end{array}$

- Anzahl 51

53

50

52

Probanden

$66,1(3,4)$

$67,8(4,1)$

$67(3,2)$

$66,1(4,1)$

Mittelwert, 1. Teil

der Untersuchung

(= Vorlesen)

in $\mathrm{dB}$ (SD)

- Stimmlautstärke-

Mittelwert, 2. Teil

$62,6(3,2)$

$64,7(3,8)$

$63,5(2,9)$

$61,9(3,4)$

der Untersuchung

(= Interview)

in $\mathrm{dB}$ (SD)

- Stimmlautstärke-

Mittelwert, 1. +

$64,4(3)$

$66,3(3,7)$

$65,2(2,7)$

$64(3,4)$

2. Teil der

Untersuchung

in $\mathrm{dB}(\mathrm{SD})$

Die Hypothese, dass sich die Sprechstimmenlautstärke von Medizinern im Vergleich zu Nichtmedizinern nach 5 Jahren Studium signifikant erhöht, wurde mit Hilfe einer zweifaktoriellen Varianzanalyse geprüft. Varianzanalysen erfordern eine Normalverteilung der abhängigen Variablen, in diesem Fall der Sprechstimmenlautstärke. Aus den beiden Stimmuntersuchungen wurde für jeden Probanden/jede Probandin ein durchschnittlicher Stimmintensitätsmittelwert bestimmt. Um die Datenverteilung der Stimmlautstärken grafisch darzustellen, wurde für jede Untersuchungsgruppe ein Boxplot erstellt. Zur Überprüfung und Veranschaulichung der Normalverteilung wurden alle vier Boxplots in einem Diagramm gegenübergestellt (Abb. 2). Daraus konnte man erkennen, dass sich die Studiendaten symmetrisch um den Median verteilen, so dass von einer Normalverteilung auszugehen war. Die Stimmlautstärke der Probanden konnte demnach in einer zweifaktoriellen Varianzanalyse als abhängige Variable verwendet werden. Die Variablen ,Semesterzahl‘ 
(1. Semester versus $\geq 9$. Semester) und ,Sudienfach' (Mediziner versus Nichtmediziner) lieferten die unabhängigen Variablen für die Analyse. Für die unabhängigen Variablen und deren Interaktion wurden über eine F-Statistik die entsprechenden $p$-Werte bestimmt. Die beiden unabhängigen Variablen einzeln betrachtet wirkten sich nicht signifikant auf die Stimmlautstärke der Studenten aus (Semesterzahl: $p=0,470$; Studienfach: $p=0,126)$. Jedoch ergab sich aus der Interaktion der unabhängigen Variablen (Semesterzahl $x$ Studienfach) ein hochsignifikanter Einfluss auf die Lautstärke der Studentenstimmen $(p=0,001)$. In Abbildung 3 werden die Ergebnisse der Varianzanalyse grafisch dargestellt.

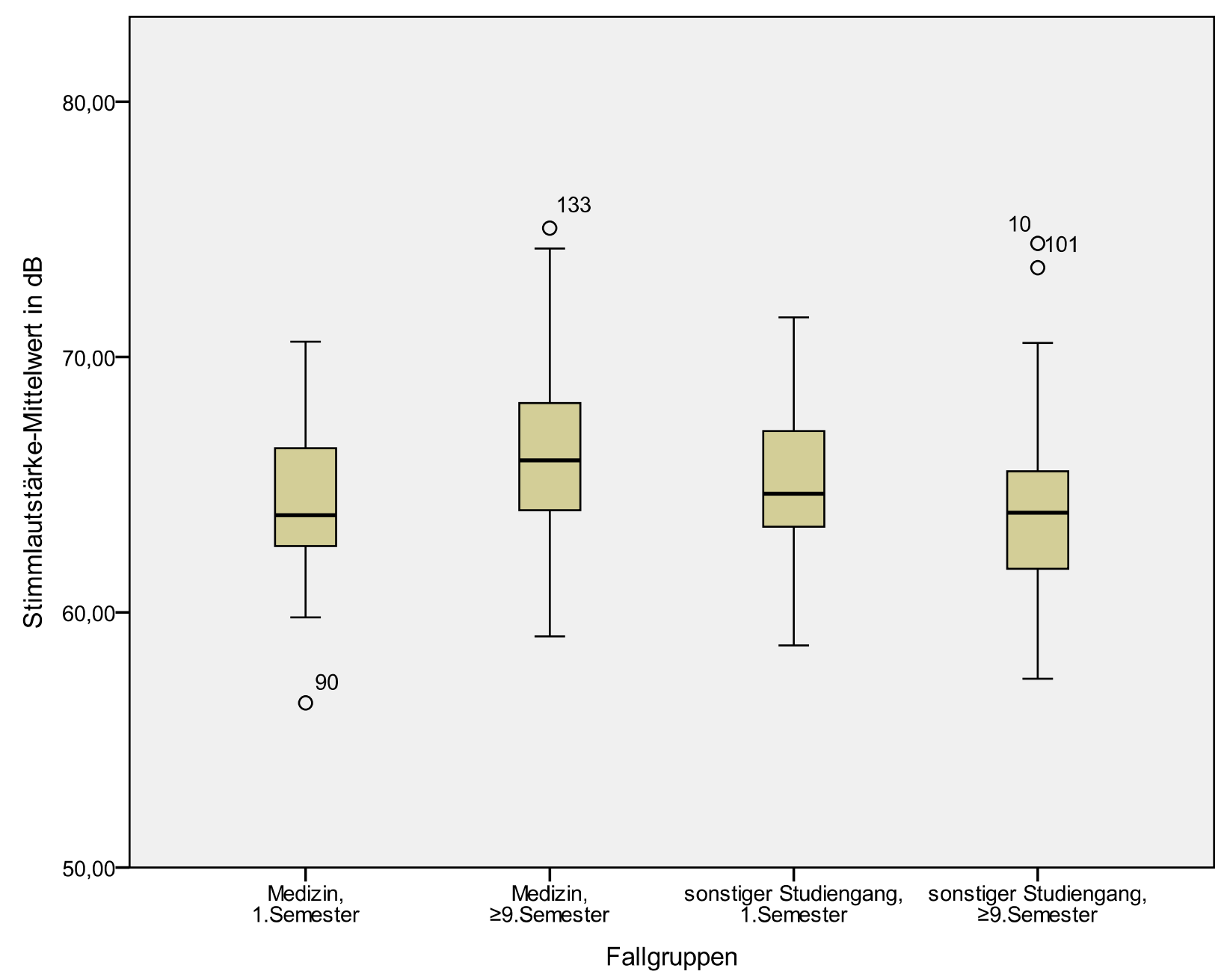

Abbildung 2: Boxplots der vier Fallgruppen 


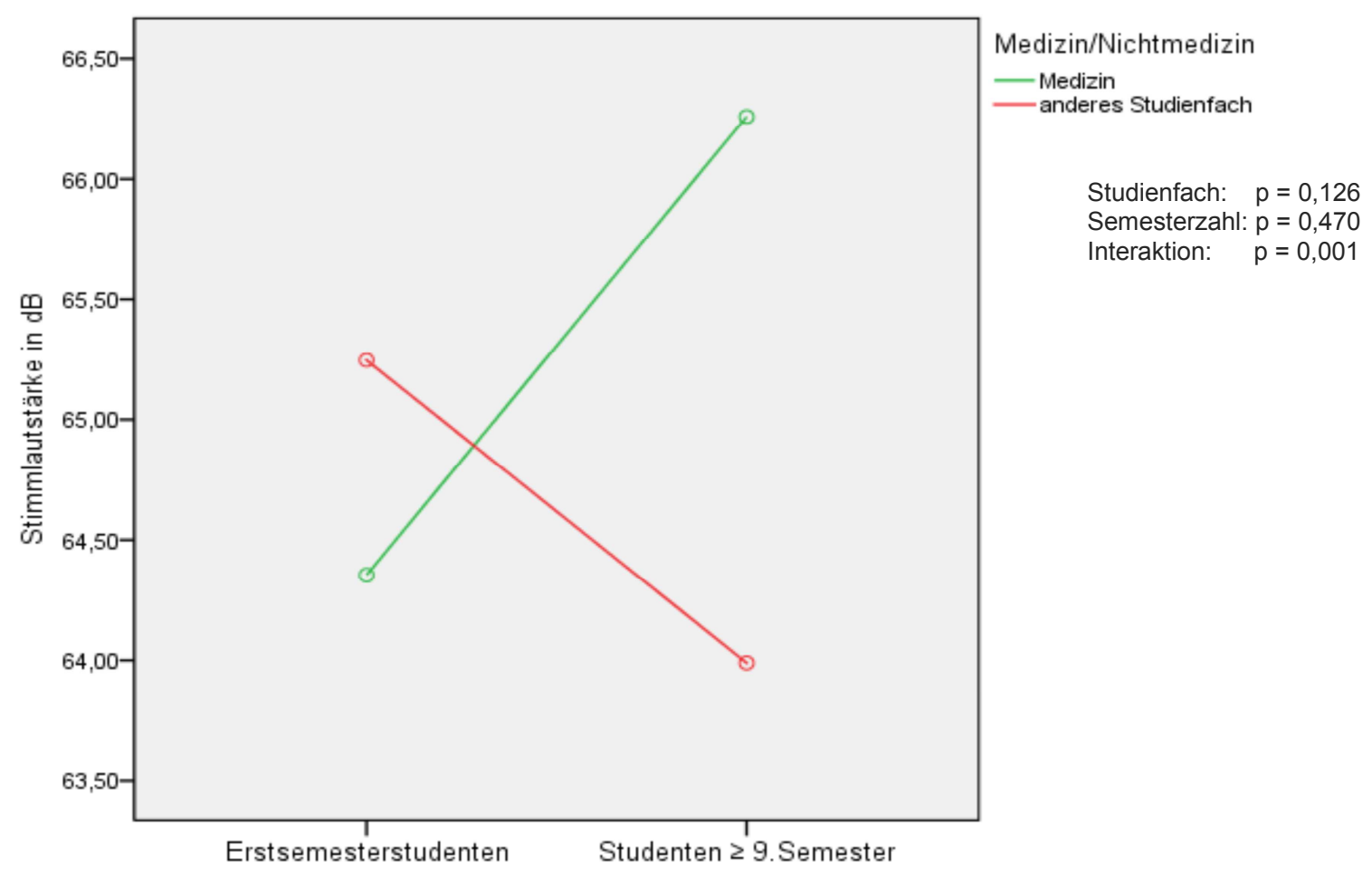

Abbildung 3: Ergebnisse Varianzanalyse

\subsection{Einflussfaktoren auf die Stimmlautstärke}

Unabhängig von Semesteranzahl und Studienrichtung wurde untersucht, ob es weitere Einflussfaktoren auf die Lautstärke der Stimme gibt. Die erhobenen Daten wurden hierfür zunächst unter theoretischen Gesichtspunkten betrachtet und mögliche Einflussgrößen extrahiert. Die Verfasserin wählte für die Untersuchung die insgesamt sechs folgenden Variablen aus:

- Braucht der Proband/die Probandin seine/ihre Sprechstimme für den Beruf?

- Lebt im Umfeld des Probanden/der Probandin ein Schwerhöriger?

-Wie schätzt der Proband/die Probandin die eigene Gesprächigkeit ein?

- T-Wert aus dem Interaktions-Angst-Fragebogen (IAF)

- Gesamtscore des Stimmstörungsindex (SSI)

- Stanine aus dem Freiburger Persönlichkeitsinventar (FPI-R)

Zur Untersuchung der potentiellen Einflussfaktoren erfolgten Regressionsanalysen mit Rückwärtsausschlussverfahren. Als Ergebnis dieser Analysen zeigte sich, dass die Daten des SSI und des IAF einen signifikanten Einfluss auf die Stimmlautstärke nahmen. Die anderen Variablen wurden von dem verwendeten Verfahren ausgeschlossen, da sie eine geringe Teilkorrelation mit der Stimmlautstärke zeigten und 
der zugehörige Regressionskoeffizient nicht signifikant war. Für die Korrelation des SSI liegt der $p$-Wert bei 0,025 (T-Wert=-2,251) und für die Korrelation des IAF wurde ein $p$ Wert von 0,036 (T-Wert=-2,114) ermittelt. Das Bestimmtheitsmaß $R^{2}$ ist bei einem Wert von 0,070 als sehr gering zu interpretieren $\left(R=0,264\right.$; korrigiertes $\left.R^{2}=0,061\right)$. Lediglich $7 \%$ der Streuung der Stimmlautstärke lassen sich mit den Variablen ,Score SSI' und ,TWert IAF' im Regressionsmodell erklären. In Anbetracht der mangelnden Güte des Regressionsmodells wurde auf weiterführende Analysen verzichtet. 


\section{Diskussion}

Anhand von objektiven Stimmfeldmessungen mit Hilfe des Software-Programmes ,lingWAVES Stimmfeld Plus' und mit dem dazugehörigen Schallpegelmesser konnten wir signifikante Stimmlautstärkeunterschiede zwischen Medizinstudenten im ersten Semester und Medizinstudenten am Ende ihres Studiums nachweisen. Die Mediziner im höheren Semester redeten signifikant lauter als die Mediziner im ersten Semester. Die Hypothese, dass die Sprechstimme von Medizinern im fortgeschrittenen Studium im Vergleich zu gleichaltrigen Studenten anderer Fachrichtungen signifikant lauter ist, konnte ebenfalls bestätigt werden. Die Forschungsergebnisse lassen sich auf beide Stimmuntersuchungen der Studie übertragen. Sowohl beim Vorlesen eines Standardtextes als auch im Interview werden die Mediziner beim Sprechen lauter und die Nichtmediziner leiser. Beide Stimmuntersuchungen der Studie in Betracht ziehend, ergibt sich bei den Medizinern eine Zunahme der Stimmlautstärke um 1,9dB und bei den Nichtmedizinern eine Abnahme der Stimmlautstärke um 1,2dB. Als weiteres Ergebnis dieser Studie wird die Lautstärke der Stimme signifikant davon beeinflusst, wie selbstsicher/dominant ein Student ist (Einflussfaktor: T-Wert aus dem InteraktionsAngst-Fragebogen). Zusätzlich wirken sich stimmliche Handicaps negativ darauf aus, wie laut ein Proband/eine Probandin spricht (Einflussfaktor: Score des SSI).

\subsection{Methodik}

Die vorgelegte Studie wurde nach dem Prinzip einer Querschnittsstudie durchgeführt. Durch soziodemografische Parallelisierung waren quasi-longitudinale Vergleiche zwischen Studenten des 1. und des 9./10. Fachsemesters zulässig. Das empirisch bessere Forschungsdesign wäre eine Längsschnittuntersuchung gewesen. Jedoch ist es logistisch schwer möglich, bei denselben Studenten, die im ersten Studiensemester an der Studie teilgenommen hätten, nach 5 Jahren Studium eine erneute Untersuchung durchzuführen. Beispielhaft sollen hier ein paar wenige Gründe genannt werden, welche die Durchführung einer Längsschnittstudie erschwert hätten:

Aus zwei notwendigen Erhebungen resultiert ein höherer zeitlicher Aufwand für die Studienteilnehmer. Zwischen der ersten und der zweiten Erhebung liegt ein Zeitintervall von 5 Jahren. Danach muss mit einer erschwerten Kontaktierung der Probanden gerechnet werden. Schwierigkeiten bei der Kontaktaufnahme können unter anderem entstehen durch Umzug des Probanden, Studienortwechsel, eventuellen Abbruch des Studiums oder auch Interessensverlust an der weiteren Studienteilnahme. Nicht zuletzt soll erwähnt werden, dass bei einer Latenzzeit von 5 Jahren ein großer zeitlicher Studienrahmen erforderlich ist. 


\subsubsection{Messinstrumente}

\subsubsection{Technische Instrumente}

Viele der häufig verwendeten Mikrofone erfüllen nicht die notwendigen technischen Voraussetzungen, um zuverlässig Lautstärken zu messen (Svec and Granqvist 2010). In der Stimmstudie wurde mit der ,lingWAVES'-Software, einem handelsüblichen Laptop und dem ,lingWAVES'-Schallpegelmesser gearbeitet. Der ,lingWAVES'Schallpegelmesser besitzt ein integriertes Mikrofon, welches objektiv Lautstärkewerte erfasst. Es ergaben sich im gesamten Studienverlauf keine technischen Fehlerquellen; alle stimmlichen Messungen konnten für die Datenauswertung verwendet werden. Als standardisierte Messinstrumente finden die Hard- und Software von ,lingWAVES' auch im klinischen Alltag zur Diagnostik und Verlaufskontrolle von Stimmstörungen Verwendung (Nawka et al. 2006).

\subsubsection{Vorlesen und Interview}

Es gibt verschiedene Möglichkeiten für stimmliche Aufgaben, bei denen die Stimme einer Person aufgezeichnet werden kann. Von Zraick und seinen Mitarbeitern wurde der Einfluss der Untersuchungsaufgabe auf die Lautstärke von Stimmen erforscht (Zraick et al. 2004). Dabei wurde der Effekt von vier verschiedenen Methoden verglichen (1. Rückwärtszählen, 2. Bildbeschreibung, 3. Vorlesen, 4. spontane Beschreibung des Raumes, in dem sich der Teilnehmer befand). Zraick et al. haben beweisen können, dass die für eine Studie gewählte, stimmliche Aufgabenstellung an einen Probanden einen signifikanten Einfluss auf die daraus ermittelte Stimmlautstärke mit sich bringt (Zraick et al. 2004). Um den Effekt der Aufgabenstellung zu minimieren, wurden in der Stimmstudie zwei Methoden angewandt (Vorlesen und Interview) und für die weiterführenden Analysen ein durchschnittlicher Dezibelwert aus beiden Methoden ermittelt.

Beide Methoden der Stimmstudie - sowohl Vorlesen als auch Interview - waren nicht nur in der Durchführung, sondern auch inhaltlich vergleichbar. Der Text für das Vorlesen und die Inhalte des Interviews erforderten genaue Vorgaben. Ein vorzulesender Text und gestellte Fragen lösen bei Probanden Emotionen aus. Emotionen beeinflussen die Atmung und Sprechweise von Menschen; sie können beispielsweise mit einen Anstieg oder eine Abnahme der Stimmenlautstärke einhergehen (Heim et al. 1968, Siegman et al. 1990). Aus diesem Grund wurde festgelegt, dass alle Probanden den gleichen Text vorlasen. Weiterhin wurden von den Untersuchern während des Interviews im Vorfeld festgelegte Fragen gestellt. Die Fragen waren die gleichen, sie wurden jedoch individuell für jeden Probanden passend formuliert, zum Beispiel Frage 1: „Warum studierst du Medizin?" (so formuliert bei Medizinstudenten, bei Studenten anderer 
Fachrichtungen wurde hier das zutreffende Studium benannt, Vgl. detaillierter Kap. 4.4, S. 15-16). Außerdem musste für den Fall, dass die Antworten eines Probanden zu kurz formuliert waren, eine beliebige Zusatzfrage gestellt werden. Diese Form der Befragung wird als halbstandardisiertes Interview bezeichnet.

Es gibt letztendlich nur grundsätzliche Vorstellungen darüber, wie ein bestimmter Text auf Menschen wirkt und welche Themen bei Probanden als relativ unproblematisch angesprochen werden können. Der Text ,Nordwind und Sonne` ist ein in der Phonetik und Linguistik viel verwendeter Standardtext und daher auch im Handbook of the International Phonetic Association zu finden (International Phonetic Association 1999, S. 89). Als bewährtes Forschungsinstrument fand dieser Text Verwendung in der Stimmstudie. Das Thema ,Studium' wurde bei der Studienplanung für den Interviewteil ausgewählt. Es wurde als nicht zu persönlich erachtet für ein kurzes Interview mit einem fremden Untersucher. Was der auserwählte Text und die formulierten Fragen zum Studium individuell bei einem Probanden für Emotionen und damit für Auswirkungen auf dessen Stimmlautstärke haben, lässt sich nicht ausreichend beschreiben.

\subsubsection{Fragebögen}

Verwendet man in einer Studie Fragebögen, so wirft das verschiedene Problematiken auf. Speziell Fragebögen, die Charaktereigenschaften und Persönlichkeitsstrukturen erfassen - wie auch der IAF oder das FPI-R - ,bergen die Gefahr, dass die Probanden die Antworten gezielt auswählen, um sich positiv darzustellen. Die Items lassen häufig erkennen, welche Inhalte sie verfolgen. Davon kann das Antwortverhalten der Probanden beeinflusst werden. In diesem Kontext findet man in der Literatur unter anderem den Begriff „soziale Erwünschtheit“ (u.a. Fahrenberg et al. 2001, S. 40; Becker 1997, S. 51). Der Befragte möchte hierbei eigene sozial unerwünschte Einstellungen nicht preisgeben und/oder sozial erwünschte Einstellungen vortäuschen (Scholl 2009). In der Stimmstudie kamen neben dem FPI und dem IAF die Fragebögen der HADS und des SSI zum Einsatz. Überlegungen zu dem Gesichtspunkt der sozialen Erwünschtheit ließen sich auf die HADS und den SSI übertragen. Es ist jedoch anzunehmen, dass insbesondere die Themen des FPI-R (Aggressivität) und des IAF (Maß an Selbstsicherheit) Probanden dazu verleiten könnten, nicht so zu antworten, wie es der individuellen Ansicht entspricht, sondern so, wie es gesellschaftlich geschätzt wird. Demnach wird bei den Ergebnissen des FPI-R und des IAF die größte Wahrscheinlichkeit für verzerrte Ergebnisse im Zusammenhang mit der sozialen Erwünschtheit vermutet:

Das FPI-R kann Probanden im Sinne der sozialen Erwünschtheit Schwierigkeiten bereiten. Eine Aussage des FPI-R, wie zum Beispiel, Wenn ich Zuflucht zu körperlicher Gewalt nehmen muss, um meine Rechte zu verteidigen, so tue ich es', kann bei der 
Beantwortung zur Diskrepanz zwischen persönlichen Impulsen und moralischen Vorstellungen führen. In der Stimmstudie beantworteten die Studenten weiterhin die IAF-Skala ,Angst vor Selbstbehauptung'. Diese erfasst das Maß an Selbstsicherheit und Selbstbewusstsein einer Person. Zurückhaltende Menschen empfinden häufig Scham für ihre geringe Selbstsicherheit. Aus diesem Grund ist es denkbar, dass zurückhaltende Probanden die IAF-Items so beantwortet haben, wie sie scheinbar gesellschaftlich hoch anerkannt werden.

Es gibt Testverfahren beziehungsweise Kontrollskalen, welche die Tendenz eines Probanden, sozial erwünschte Antworten anzugeben, erfassen sollen. Erreicht ein Proband in solch einem Test einen hohen Testwert, so können möglicherweise andere Fragebogenergebnisse dieses Probanden nicht valide interpretiert werden. Becker et al. sind bei der Erarbeitung des IAF auf diese vermeintliche Validitätsminderung eingegangen. Dafür wurde untersucht, inwieweit die IAF-Skalen mit verschiedenen Kontrollskalen zur sozialen Erwünschtheit korrelieren. Dabei kamen drei verschiedene Kontrollverfahren zum Einsatz: die Marlowe and Crowne Social Desirability Scale (MCSDS), die Social Desirability Scale aus der Personality Research Form (PRF-Dy) und die Offenheitsskala des FPI (Becker 1997). „Das Ergebnis ist eindeutig: Keiner der Korrelationskoeffizienten weicht signifikant von Null ab. So erfreulich dieses Ergebnis ist, so wenig schließt es aus, dass im Einzelfall bzw. unter besonderen Untersuchungsbedingungen Abwehrhaltungen zum Tragen kommen“ (Becker 1997, S. 52). Demgegenüber erachten Fahrenberg et al. Korrelationsstudien zur Erklärung von Antworttendenzen als „kaum geeignet“ (Fahrenberg et al. 2001, S. 41). Der Einfluss der sozialen Erwünschtheit bleibt weiterhin Gegenstand wissenschaftlicher Diskussionen und kann für die dargelegte Studie nicht ausreichend beurteilt werden.

Auch die Akquieszenz (sogenannte Bejahungstendenz) sollte bei der Verwendung von Fragebögen diskutiert werden. Akquieszenz beschreibt das Phänomen, dass manche Menschen bei der Beantwortung von Fragebögen eher dazu tendieren, den Items zuzustimmen. Dabei wird der Inhalt der Fragestellung unkritisch betrachtet (Moosbrugger und Kelava 2008). Die Tendenz zum Bejahen wird mit unterschiedlichen Charaktereigenschaften einer Person in einen Kontext gebracht. So sollen beispielsweise Menschen mit gering ausgeprägter Ich-Stärke zur Akquieszenz neigen (Schnell et al. 2008). Insbesondere das FPI-R ist mit seinen binären Antwortmöglichkeiten ,stimmt' und ,stimmt nicht' so konstruiert, dass Probanden zu einseitig antworten könnten.

Im Gegensatz zum FPI-R bieten die Fragebögen des SSI, des IAF und der HADS zu jedem Item Likert-Skalen. Somit kann der Proband die Ausprägung seiner Antworten zu den Items abstufen. Mehrstufige Skalen können Probanden dazu verleiten, Antworten des mittleren Bereiches zu wählen und extreme Ausprägungen zu vermeiden. 
Wissenschaftlich wird diese Beobachtung mit dem Terminus ,Tendenz zur Mitte beschrieben (Kühn et al. 2006). Unsicherheit und Unwissenheit fördern die Tendenz zur Mitte. Die Skala des SSI ist fünfstufig (von $0=$ nie bis 4=immer), die des IAF siebenstufig (von 1=ziemlich angenehm bis $7=a ̈ u ß e r s t ~ u n a n g e n e h m$ ) und die Skala des HADS vierstufig (verschiedene Beschreibungen zu jedem Item). Somit muss eine potentielle Ergebnisverzerrung durch die Tendenz zur Mitte berücksichtigt werden. Eventuell auffällige Probanden, denen in der Regel besonderes Interesse bei Fragebogenanalysen gilt, wurden aus diesem Grund womöglich nicht erfasst.

Die Phänomene ,soziale Erwünschtheit', ,Akquieszenz' und ,Tendenz zur Mitte' können zu einer Datenverzerrung führen und müssen bei der Interpretation der erbrachten Studienergebnisse einkalkuliert werden.

Der IAF setzt sich aus 6 Basisskalen zusammen. Es ist für gewöhnlich vorgesehen, dass ein Proband den gesamten IAF beantwortet. Daraus soll die allgemeine Angstneigung einer Person und darüber hinaus die Angstneigungen auf spezielle Bereiche bezogen ermittelt werden. Für die Stimmstudie war eine der Basisskalen, die Skala zur Selbstbehauptung, von besonderem Interesse und wurde separat zur Befragung gestellt.

Aus dem FPI wählte man zum Studienzweck die Subskala zur Aggressivität aus und ließ sie von den Probanden beantworten. Insgesamt aber besteht das FPI aus 12 Subskalen und ist so konzipiert, dass eine Person alle Skalen in einem Fragebogen beantwortet. Sowohl beim FPI als auch beim IAF gibt es Interkorrelationen zwischen den verschiedenen Skalen. Auf den IAF bezogen gilt Folgendes: "Mittelt man die Interkorrelation zwischen den 6 Basisskalen unter Zugrundelegung der zTransformation, so ergibt sich ein Wert von .30. Bezogen auf die Reliabilität dieser Skalen liegt die mittlere Interkorrelation einerseits so niedrig, dass sich die gesonderte Erfassung verschiedener bereichsspezifischer Angstneigungen lohnt und rechtfertigen lässt, und andererseits so hoch, dass am Konstrukt einer "globalen Angstneigung“ festgehalten werden kann" (Becker 1997, S. 31). Trotzdem sollte dem Sachverhalt, dass der IAF und das FPI nur partiell in dem Fragebogenteil der Stimmstudie verwendet wurden, bei der Interpretation Beachtung geschenkt werden. Die kompletten Fragebögen des IAF und des FPI zu erheben, hätte den zeitlichen Rahmen der Studienteilnahme überschritten. Zudem galt das Interesse explizit den auserwählten Skalen. Ergänzend sei erwähnt, dass sowohl für den IAF als auch für das FPI Normtabellen zur Verfügung gestellt werden, welche die Auswertung einzelner Skalen erlauben (Becker 1997, Fahrenberg et al. 2001). Da bei dem SSI und der HADS die kompletten Fragebögen von den Probanden beantwortet wurden, erübrigt sich hierfür die Diskussion zur Interpretation von Subskalen. 


\subsubsection{Messsituation}

Die von Nawka et al. empfohlenen Untersuchungsbedingungen wurden erfüllt, die Umgebungslautstärke lag stets unter $40 \mathrm{~dB}$ und ein $30 \mathrm{~cm}$-Abstand zwischen Proband und Schallpegelmesser wurde eingehalten (Nawka et al. 2006). Nawka et al. schlagen weiterhin vor, die Stimmaufnahme in stehender Position der Probanden durchzuführen (Nawka et al. 2006). In der Stimmstudie wurde bevorzugt, dass die Probanden in entspannter Haltung dem Untersucher in einem festgelegten Abstand gegenübersaßen. So hofften wir, eine übliche Gesprächsatmosphäre zu schaffen und Stimmlautstärken zu messen, mit denen der Proband/die Probandin auch im Alltag spricht. Dem Studienteilnehmer wurde vor Beginn der Untersuchung erläutert, dass Parameter seiner Stimme aufgezeichnet werden. Der Begriff ,Lautstärke' wurde bewusst nicht erwähnt, um eine Beeinflussung diesbezüglich zu vermeiden. Auch wurde vom Untersucher erklärt, dass der Inhalt des Gesprochenen sofort gelöscht wird und nicht reproduzierbar ist. Der Proband sollte möglichst frei sprechen, ohne sich zu sehr auf den Inhalt des Gesagten zu konzentrieren und dadurch eventuell von seiner gewöhnlichen Sprechstimmenlautstärke abzuweichen.

\subsection{Ergebnisse}

\subsubsection{Forschungskontext}

Die Stimmstudie liefert Erkenntnisse zur Stimmentwicklung von Medizinstudenten - auch im Vergleich zu Studenten anderer Fachrichtungen - und rechtfertigt Rückschlüsse auf Sozialisationsprozesse während des Medizinstudiums. Sozialisation und Aneignung einer professionellen Identität im Medizinstudium wurde in einer großen Anzahl medizinsoziologischer Studien untersucht (u.a. Lingard et al. 2003, Irvine 1999, Pitkala and Mantyranta 2003, Radcliffe and Lester 2003). Leider fehlt es hierbei an quantitativen Messwerten.

Das Thema ,nonverbales Gesprächsverhalten' hat in der Vergangenheit gleichermaßen für zahlreiche Studien Forschungsgrundlage gebildet und ist auch weiterhin Gegenstand intensiver Forschung. Die Besonderheit des Arzt-Patienten-Gesprächs führt dazu, dass nonverbales Verhalten insbesondere im medizin-kommunikativen Kontext untersucht wurde, sowohl bereits bei Studierenden der Medizin (u.a. Haber and Lingard 2001, Raz and Fadlon 2006, Zick et al. 2007) als auch bei praktizierenden Ärzten im klinischen Altag (u.a. Harrigan et al. 1989, Schmid Mast et al. 2008). In der Stimmstudie war vor allem von Interesse, inwieweit sich nonverbales Gesprächsverhalten und dabei explizit die Stimme von Medizinstudenten im Studium entwickelt. Studenten können nonverbale Fähigkeiten, die einen gewissen Professionalismus präsentieren, bereits innerhalb kurzer Zeit erlernen. Hottel und Hardigan konnten zeigen, dass Studierende der 
Zahnmedizin bereits nach einem 35stündigen Kurs zum Thema nonverbaler Kommunikation signifikante Verbesserungen in diesem Bereich zeigten. Beobachtungen von Patientengesprächen vor und nach dem Kurs führten unter anderem zu dem Ergebnis, dass sich das professionelle Auftreten der Studenten durch den Kurs signifikant verstärkte (Hottel and Hardigan 2005). Die Stimme und explizit ihre Lautstärke wurden in Studien zum nonverbalen Gesprächsverhalten nur marginal betrachtet.

\subsubsection{Interpretation der erhobenen Stimmlautstärken}

Um die erhobenen Stimmlautstärken zu interpretieren, bedarf es gewisser Vergleichswerte. Die Stimmlautstärke war für die psychologische Forschung der 70er bis 90er Jahre von großer Bedeutung. Es erfolgten quantitative Messungen der Stimmlautstärke und Schlussfolgerungen in Bezug auf Persönlichkeitsmerkmale wurden erstellt. Die Stimme wurde untersucht im Zusammenhang mit den Charaktereigenschaften Durchsetzungsfähigkeit, Bestimmtheit und Selbstbehauptung (,assetiveness'), Dominanz, Selbstvertrauen und Extraversion.

\subsubsection{Laute Stimme und Durchsetzungsfähigkeit}

In Zusammenarbeit mit verschiedenen Kollegen erforschte Kimble, wie durch die menschliche Stimme Durchsetzungsfähigkeit und Selbstvertrauen vermittelt werden kann. Kimble und Musgrove führten 1988 eine Studie durch, welche einen Zusammenhang erstellte zwischen Durchsetzungsfähigkeit und Stimmlautstärke von Psychologiestudenten/innen. 250 Psychologiestudenten/innen erklärten sich dafür bereit, den Rathus Assertiveness Schedule auszufüllen. Dieser Fragebogen erstellt einen Index für die Durchsetzungsfähigkeit und Dominanz einer Person. Diejenigen Studenten, die besonders durchsetzungsfähig waren, und diejenigen, die wenig durchsetzungsfähig waren, selektierte man aus den 250 ausgefüllten Fragebögen heraus. Die ausgewählten 128 Studenten (64 weibliche und 64 männliche Personen) führten paarweise fünfminütige Gespräche zu einem vorgegebenen Thema. Die Gespräche wurden gefilmt, mit Mikrofonen aufgenommen und später zu Studienzwecken analysiert. Kimble und Musgrove konnten zeigen, dass durchsetzungsfähige Menschen lauter und mehr reden als Menschen mit wenig Durchsetzungsvermögen. Ergänzend wurde erkannt, dass im Verlauf der Gespräche die Stimmlautstärken der Gesprächspartner signifikant anstiegen. Betrachtet man alle 128 untersuchten Psychologiestudenten von Kimble und Musgrove zusammen, so ergab sich zu Beginn der Konversation ein durchschnittlicher Lautstärkewert von 63,4dB und nach knapp 5 Minuten Gesprächsdauer bereits ein durchschnittlicher Wert von 64,2dB (Kimble and Musgrove 1988). 
Die Stimmaufnahmen der Stimmstudie waren von relativ kurzer Dauer. Es ist nach den Erkenntnissen von Kimble und Musgrove anzunehmen, dass die durchschnittlichen Lautstärkewerte unserer Probanden bei länger dauernden Stimmaufnahmen lauter berechnet worden wären. Die durchschnittliche Lautstärke der Medizinerstimmen im höheren Semester lag in der vorgelegten Studie bei 66,3dB. Trotz kurzer Redezeit liegt dieser Wert deutlich über den Durchschnittswerten der Psychologiestudenten in der Studie von Kimble und Musgrove - sowohl zu Beginn als auch gegen Ende der analysierten Gespräche. Daraus lässt sich ableiten, dass die Medizinstudenten der Stimmstudie über ein großes Maß an Durchsetzungsvermögen verfügen. Diese Schlussfolgerung deckt sich mit den Fragebogenergebnissen der Stimmstudie, wonach die Medizinstudenten am Ende ihres Studiums den höchsten FPI-Mittelwert aufweisen und somit die Gruppe mit dem größten Durchsetzungsvermögen bilden.

\subsubsection{Laute Stimme und Selbstvertrauen}

In Zusammenarbeit mit Seidel untersuchte Kimble 1991, ob die Stimmlautstärke ein Indiz dafür darstellt, wie groß das Selbstvertrauen einer Person ist. Über einen Computermonitor wurden 101 Probanden standardisierte Allgemeinwissensfragen gestellt. Die Probanden antworteten in ein Mikrofon, wodurch ihre Stimmlautstärken gemessen werden konnten. Unmittelbar nach jeder Frage sollten die Teilnehmer auf einer siebenstufigen Skala angeben, wie zuversichtlich sie sind, die Frage richtig beantwortet zu haben. Die Hypothese, dass Menschen lauter reden, wenn sie ein großes Selbstvertrauen besitzen, konnte bestätigt werden. Die Ergebnisse von Kimble und Seidel waren signifikant $(p<0,05)$. Die Lautstärkedaten dieser Studie wurden nicht in Dezibelwerten angegeben, sondern man verglich die Daten anhand von Stimmsignalamplituden (Kimble and Seidel 1991). Somit ist ein direkter Vergleich der durchschnittlichen Lautstärken mit unseren Ergebnissen nicht möglich. Nach den

Studienresultaten von Kimble und Seidel impliziert eine Stimmlautstärkezunahme der Medizinstudenten auch eine Zunahme ihres Selbstvertrauens.

\subsubsection{Laute Stimme und Extraversion}

Scherer KR (1978) nahm 24 amerikanische Männer auf Tonbandgerät auf, während diese simulierte Jurydiskussionen führten. Nach den Diskussionen bewerteten sich die Teilnehmer selbst und jeweils drei weitere Sprecher bezüglich ihrer Stimmmerkmale und Persönlichkeitseigenschaften. Im nächsten Schritt wurden die Tonbandaufnahmen von unabhängigen Studentinnen und Phoniatern beurteilt beziehungsweise analysiert. Möglichst viele unabhängige Gruppen sollten die Stimmen der Studienteilnehmer einschätzen, um die Objektivität der Ergebnisse zu erhöhen. Die Objektivität dieser Studie war eingeschränkt, da die Stimmen anhand von Tonbandaufnahmen und siebenstufigen Skalen beurteilt wurden. Dezibelwerte zum Vergleich mit den Werten 
unserer Studie fehlen. Scherer KR bestimmte mit Hilfe von Korrelationskoeffizienten nach Pearson den Zusammenhang zwischen Stimme und Extraversion eines Menschen. Aus den Studienergebnissen erstellte er ein Modell, welches anhand von stimmlichen Parametern den Grad der Extraversion eines Menschen erklärt. Demnach korrelieren Lautstärke und Schärfe in der Stimme positiv mit Extraversion und eine dunkle Stimme mit deutlich hörbaren Atemgeräuschen (,breathiness') korreliert negativ mit Extraversion (Scherer KR 1978, S. 478). Die Stimmstudie hat zeigen können, dass Medizinstudenten im höheren Semester eine signifikant lautere Stimme kennzeichnet als Medizinstudenten im ersten Semester und als gleichaltrige Studenten anderer Fachrichtungen. Vergleicht man Scherers Ergebnisse mit denen der Stimmstudie, so lässt sich schlussfolgern, dass Medizinstudenten am Ende ihres Studiums auch extrovertierter sind als Nichtmediziner im fortgeschrittenen Studienabschnitt und als Medizinstudenten zu Beginn des Studiums. Extraversion bildet einen Bestandteil der erwünschten professionellen Identität von Medizinern, die Anforderungen des ,hidden curriculums' werden erfüllt.

Im Vergleich der Stimmstudie mit Studien zu Stimme und Persönlichkeit lässt sich zusammenfassen, dass all die Charaktereigenschaften, die mit einer lauten Stimme in Verbindung gebracht werden - Durchsetzungsfähigkeit, Selbstvertrauen und Extraversion - zu der professionellen Identität von Medizinstudenten gehören. Dass die Lautstärke von Medizinerstimmen signifikant zunimmt, zeigt, dass Medizinstudenten folglich auch durchsetzungsfähiger, selbstbewusster und extrovertierter werden. Lautstärke in der Stimme kann demnach als ein messbarer Parameter für den Grad von Professionalisierung im Medizinstudium betrachtet werden.

\subsubsection{Veränderungen der Stimmlautstärke}

Die Stimmstudie verfolgte das Ziel, Veränderungen der Stimmlautstärke von Studenten aufzudecken. Dabei stellten das Studienfach und die Semesteranzahl die bestimmenden Einflussgrößen dafür dar, wie sich die Lautstärke der Stimmen entwickelte. Wie im vorherigen Kapitel erläutert, kann man anhand einer lauten Stimme auf Persönlichkeitseigenschaften Rückschlüsse ziehen. Neben der Bedeutung von Persönlichkeitsmerkmalen für Stimmparameter gibt es nur wenige Untersuchungen zu Einflussfaktoren, welche die Stimmlautstärke von Menschen tatsächlich verändern.

Södersten et al. untersuchten 2005, wie sich Umgebungsgeräusche auf die Stimmlautstärke auswirkten. Die schwedische Studie von Södersten et al. ist inhaltlich mit dem Hintergrund der Stimmstudie nur wenig vergleichbar. Jedoch ähnelt sie vom Untersuchungsdesign dem Studienaufbau unserer Studie und liefert objektiv vergleichbare Werte. 
Der Lombard-Effekt erklärt den Vorgang, dass Menschen bei lauter Umgebung ihre Stimmlautstärke beim Sprechen erhöhen (Lane and Tranel 1971). Södersten et al. beschrieben in ihrer Arbeit, dass Transportfahrzeuge (wie zum Beispiel Züge) zu einem Umgebungslautstärkepegel von etwa $74 \mathrm{~dB}$ führen und diese Umgebung als subjektiv sehr laut empfunden wird. Reden Menschen bei diesen Umgebungsgeräuschen, so führt das zu einem Anstieg der Stimmlautstärke. Gemessen wurden dabei durchschnittliche Stimmlautstärken von 66dB. Hierzu lohnt sich der Vergleich zu den Lautstärkewerten der Medizinerstimmen: Unsere Messungen ergaben bei den Medizinstudenten im höheren Semester einen durchschnittlichen Stimmlautstärkewert von 66,3dB. Die Messungen fanden in einem ruhigen Raum ohne störende Nebengeräusche statt. Das bedeutet, dass Medizinstudenten am Ende ihres Studiums in ruhiger Umgebung lauter sprechen als Menschen es üblicherweise tun, wenn sie sich innerhalb eines lauten Zuges befinden.

In Söderstens Studie wurde der Lombard-Effekt bei 23 Lehrern im mittleren Alter genauer untersucht. Sie sollten einen Text so vorlesen, dass man sie gegenüber verschiedenen simulierten Umgebungsgeräuschen verstehen konnte. Dabei wurden analog zur Stimmstudie Stimmfelder der Probanden erstellt, die Umgebungsgeräusche filterte man zur Stimmanalyse heraus. Wie zu erwarten war, hatte die Umgebungslautstärke einen signifikanten Einfluss auf die resultierende Stimmlaustärke. Beispielsweise führt eine akustische Umgebungskulisse, die einem belebten Klassenraum entspricht, zu einer Zunahme der mittleren Stimmintensität von 9-10bB. Wie bereits im Stand der Forschung erwähnt, führt eine Erhöhung der Stimmlautstärke um $10 \mathrm{~dB}$ zu einer doppelt so laut empfundenen Lautstärke für den Zuhörer (Hosse 2008). Die Ausgangsmessungen der Studie von Södersten et al. wurden in ruhiger Atmosphäre durchgeführt. Diese Messergebnisse können bezogen auf die Untersuchungsbedingungen mit den Ergebnissen der Stimmstudie verglichen werden. Die schwedischen Probanden lasen einen Text bei einem durchschnittlichen Wert von $77 \mathrm{~dB}$ vor und erbrachten damit deutlich höhere Lautstärkewerte als die Studenten in der Stimmstudie. Verschiedene Erklärungsansätze sollen dafür diskutiert werden. Das Probandenkollektiv von Södersten war mit 23 Probanden geringer als unser Kollektiv. Die 23 schwedischen Probanden waren alle Lehrer und die Stimme von Lehrern ist bedingt durch ihre berufliche Sprechsituation generell lauter (u.a. Pekkarinen and Viljanen 1991, Sala and Viljanen 1995, Simberg et al. 2005). Zudem lasen die Probanden einen Text vor, der ihnen bekannt war. Das Textmaterial wurde den Probanden im Vorfeld zugeschickt, damit sie sich für die Untersuchung damit vertraut machen konnten. Der Text hatte weiterhin Befehlscharakter; dies könnte ebenfalls dazu geführt haben, dass man inn lauter vorlas, um dem Inhalt mehr Nachdruck zu verleihen. 
Die erhobenen Werte von Södersten et al. lagen nicht nur höher als unsere Erhebungen, sondern auch deutlich höher als die Ergebnisse von Pérez Álvarez und Hacki (2000). Pérez Álvarez und Hacki erstellten Stimmfelder von 100 Probanden sowohl Männer als auch Frauen - im Alter von 19 bis 32 Jahren. Ihre Untersuchungen verfolgten den Zweck, eine Norm zu bilden für den Vergleich von Stimmfeldparametern. Auf Grund der größeren Probandenanzahl und der Altersverteilung lassen sich diese Ergebnisse mit unseren gut vergleichen. Die Studie von Pérez Álvarez und Hacki ergab einen durchschnittlichen Wert für die mittlere Sprechstimmenintensität von 65,6dB (Pérez Álvarez und Hacki 2000). Dieser Wert deckt sich sehr gut mit dem Cluster der Stimmstudie, in dem die Gruppe der normal lauten Sprecher zusammengefasst wurde. Innerhalb dieses Clusters lag der durchschnittliche Wert für die mittlere Sprechstimmenintensität bei $65,2 \mathrm{~dB}$. Weiterhin wurden für die Stimmstudie in Abhängigkeit von dem Studienfach und der Semesteranzahl der Studenten 4 verschiedene Untersuchungsgruppen gebildet. Diese 4 Gruppen wurden bezüglich ihrer Stimmlautstärke verglichen, um den Einfluss von Studienfach und Semesterzahl zu beurteilen. Vergleicht man unsere 4 Probandengruppen mit der Studiengruppe von Pérez Álvarez und Hacki, so lässt sich zusammenfassen: Nur die Mediziner im höheren Semester redeten lauter als die Probanden von Pérez Álvarez und Hacki. Für alle anderen 3 Untersuchungsgruppen wurden geringere durchschnittliche Lautstärkewerte ermittelt.

\subsubsection{Ergebnisse der Fragebögen}

In der Stimmstudie wurden ergänzend zu den Stimmfeldmessungen Daten aus standardisierten Fragebögen erhoben. Die Ergebnisse des FPI-R zeigen, dass die Medizinstudenten im ersten Semester die Gruppe mit dem geringsten durchschnittlichen FPI-R-Mittelwert (Stanine-Mittelwert=4,25) bilden. Folglich ist dies eine Studentengruppe mit tendenziell zurückhaltenden Probanden. Die Gruppe der Mediziner im fortgeschrittenen Studienabschnitt weist demgegenüber viele Studenten mit Durchsetzungsvermögen auf. Diese Gruppe hat mit einem durchschnittlichen StanineMittelwert von 4,6 den höchsten FPI-R-Mittelwert der vorgelegten Untersuchung. Der Stanine-Mittelwert für die Nichtmediziner im ersten Semester lag bei 4,42 und bei den Nichtmedizinen im fortgeschrittenen Studium bei einem durchschnittlichen Wert von 4,37. Somit sind bei den Nichtmedizinern die jüngeren Studenten im Durchschnitt durchsetzungsfähiger als ihre älteren Kommilitonen.

Die Ergebnisse des FPI-R sollen in Bezug gesetzt werden zu den Ergebnissen der Stimmfeldmessungen. Durchsetzungsfähige Menschen sprechen lauter als Menschen mit wenig Durchsetzungsvermögen (Kimble and Musgrove 1988, Vgl. detaillierter Kap. 6.2.2.1, S. 38-39). Diese These deckt sich mit den Ergebnissen der Stimmstudie. Die 
Medizinstudenten am Ende des Studiums redeten signifikant lauter und waren durchsetzungsfähiger als die Mediziner im 1. Semester. Bei den Nichtmedizinern zeigten die Erstsemesterstudenten mehr Durchsetzungsfähigkeit als die Nichtmediziner im fortgeschrittenen Studium, die Nichtmediziner im ersten Semester redeten auch signifikant lauter als die ältere Vergleichsgruppe der Nichtmediziner.

Menschen mit einem großen Selbstvertrauen reden lauter als Menschen mit weniger Selbstvertrauen (Kimble and Seidel 1991, Vgl. detaillierter Kap. 6.2.2.2, S. 39). In der Stimmstudie wurde mit Hilfe des IAF ermittelt, wie selbstsicher die Probanden sind. Von allen 4 untersuchten Probandenkollektiven waren die Mediziner im ersten Semester die Gruppe mit der geringsten Selbstsicherheit (T-Wert Mittelwert=52,9) und die Mediziner im höheren Semester die Gruppe mit der größten Selbstsicherheit (T-Wert Mittelwert=50,9). Die Erkenntnisse von Kimble und Seidel bestätigten sich in der Stimmstudie: Die selbstsicheren Mediziner im fortgeschrittenen Studium sprechen mit einer signifikant lauteren mittleren Sprechstimme als die Mediziner im ersten Semester mit weniger Selbstsicherheit.

Der psychische Gesundheitszustand von Medizinstudenten und insbesondere die Entwicklung dessen während des Studiums wurden in verschiedenen Studien untersucht. Die Zufriedenheit mit dem eigenen Leben fällt während des Medizinstudiums (Kjeldstadli et al. 2006). Diese These konnte 2006 nach einer sechsjährigen Longitudinalstudie belegt werden. Zu Beginn des Studiums sind Mediziner genauso zufrieden wie Studenten anderer Fachrichtungen. Im letzten Studienjahr liegt die Zufriedenheit bei den Medizinern signifikant unter der Zufriedenheit der gleichaltrigen Vergleichsgruppe (Kjeldstadli et al. 2006). Dyrbye et al. konnten in ihrem Review von 2006 aufzeigen, dass die psychische Belastung unter amerikanischen und kanadischen Medizinstudenten höher ist als in einer gleichaltrigen Vergleichsgruppe und als in der allgemeinen Bevölkerung. Zu Beginn des Studiums konnte kein signifikanter Unterschied bezogen auf die psychische Belastung festgestellt werden. Jedoch ist der Prozentsatz an Depressiven bei den Medizinstudenten in den späten Jahren ihres Studiums höher als bei der gleichaltrigen Vergleichsgruppe und als in der Allgemeinbevölkerung (Dyrbye et al. 2006).

Medizinstudenten sind gefährdet, ein Burnout-Syndrom zu entwickeln (u.a. Dyrbye et al. 2008, Brazeau et al. 2010, Dyrbye et al. 2010,). Santen et al. beschreiben, dass sich in ihrer Studie die Prävalenz von Burnout im Vergleich von Medizinstudenten im ersten Jahr zu Medizinstudenten im dritten Jahr verdoppelte (Santen et al. 2010). In einer aktuellen Umfrage wurden amerikanische Medizinstudenten zu ihren Belastungen im Studium befragt. Dabei wurden Daten zu den Themen Burnout, Depression, Stress, schlechte psychische Lebensqualität, schlechte physische Lebensqualität und Müdigkeit 
erhoben. In den Auswertungen zeigte sich, dass $82 \%$ der befragten Mediziner unter mindestens einer der dargelegten Belastungen litten (Dyrbye et al. 2011).

Das Thema psychische Gesundheit im Medizinstudium hat eine große Relevanz. Die dargelegten Ergebnisse der Forschungsliteratur belegen, dass das Studium der Medizin zu gesundheitlicher Beeinträchtigung führen kann. Insbesondere psychische Erkrankungen wie beispielsweise Depression oder auch Burnout stehen im Zusammenhang mit den Belastungen eines Medizinstudiums. Um diesen Aspekt aufzugreifen, wurde in der Stimmstudie die Häufigkeit von Depression und Angst innerhalb der Studienpopulation evaluiert. Als Selbstbeurteilungsfragebogen bildete die Hospital Anxiety and Depression Scale die Grundlage für die Ergebnisse. Unter allen befragten Studenten lagen die Prävalenz von Depression bei $4 \%$ und die Prävalenz von Angst bei $10 \%$. Medizinstudenten im höheren Semester waren weniger von Depression betroffen als die Medizinstudenten im ersten Semester (2\% gegenüber $4 \%$ ). Auch litten die älteren Mediziner seltener unter Angst (8\% gegenüber 14\%).

Die Medizinstudenten im ersten Semester litten prozentual häufiger an Depression und Angst als die Mediziner am Ende des Studiums. Sie redeten außerdem mit einer geringeren durchschnittlichen Stimmlautstärke als ihr älteren Kommilitonen (Stimmlautstärke-Mittelwert aus 1. + 2. Teil der Untersuchung: 64,4dB gegenüber 66,3dB). Diese Beobachtung deckt sich mit der These, dass Menschen, die zu Depression und/oder Angst neigen, in der Regel mit einer leiseren Stimme reden (Hautzinger 2003, Metzig und Schuster 2006).

Die Studienergebnisse der vorgelegten Arbeit für die Prävalenz von Depression und Angst sind als relativ niedrig zu bewerten - für die gesamte Studienpopulation (Depression: 4\%; Angst: 10\%) und insbesondere für die Gruppe der Medizinstudenten im fortgeschrittenen Studium (Depression: 2\%; Angst: 8\%). „Die Testergebnisse sind grundsätzlich mit der für Fragebogeninstrumente gebotenen Zurückhaltung zu interpretieren und sind im individuellen Einsatz nicht als diagnoseweisend sondern vielmehr als Orientierungsmarken zu verstehen“ (Herrmann-Lingen et al. 2005, S. 11). Trotz dieser Einschränkung zur Hospital Anxiety and Depression Scale liegen die Werte deutlich unter den Ergebnissen aus der Vergleichsliteratur. In einer pakistanischen Querschnittsstudie litten 46,07\% der befragten Medizinstudenten an Angst und Depression, wobei hier der politisch-gesellschaftliche Hintergrund mit zu berücksichtigen ist (Jadoon et al. 2010). In einer Befragung von mehr als 3000 amerikanischen Medizinstudenten ermittelten Dyrbye et al., dass 49\% der Studienteilnehmer depressive Symptome aufweisen (Dyrbye et al. 2007).

Medizinstudenten sind „- mehr noch als Studierende anderer Fachrichtungen - eine Risikogruppe für die Entwicklung von Depressionen, Ängsten, ,Burn-out' und anderen stressbedingten Erkrankungen“ (Kötter et al. 2011). Um diesen Entwicklungen 
vorzubeugen, gibt es protektive Faktoren. An der Universität Lübeck wird aktuell unter der Leitung von Dr. med. Thomas Kötter das ,LUST-Projekt' (Luebeck University Students Trial) durchgeführt. In dieser Studie sollen protektive Faktoren zum Erhalt der psychosozialen Gesundheit von Medizinstudenten identifiziert werden. Die Erkenntnisse des LUST-Projektes sind essenziell, um Interventionen zu schaffen, die den Gesundheitszustand der Medizinstudenten verbessern (Kötter et al. 2011).

\subsection{Ausblick}

\subsubsection{Stimmlautstärke und Stimmstörungen}

Bei andauernder, konstant hoher Stimmlautstärke muss auf die Gefahr hingewiesen werden, eine berufsbedingte Stimmstörung zu entwickeln (Titze 1999, Vilkman 2000, Verdolini and Ramig 2001). Bei Lehrern zum Beispiel bilden Stimmstörungen durch die berufliche stimmliche Belastung - explizit durch Sprechen bei hoher mittlerer Stimmintensität - ein viel untersuchtes Krankheitsbild (u.a. Russell et al. 1998, Angelillo et al. 2009, de Ceballos et al. 2011). Beruflich assoziierte Dysphonien sind auch bei Ärzten möglich, insbesondere dann, wenn Ärzte neben ihrer praktischen Arbeit zusätzlich in der Lehre tätig sind. Das Risiko, dass Ärzte durch die Verwendung hoher Stimmintensitäten eine Stimmstörung entwickeln, sollte in weiterer Forschung evaluiert werden.

In der Stimmstudie wurden mit Hilfe des Stimmstörungsindexes (SSI) die stimmlichen Handicaps der Probanden ermittelt. Dabei wurden zwischen den 4 Untersuchungsgruppen keine signifikanten Unterschiede zur Prävalenz der Handicaps erkannt $(p=0,215)$. Demnach fühlen sich Medizinstudenten durch die stimmliche Anstrengung (noch) nicht belastet. Im Gegenteil: Die Mediziner im höheren Semester bilden die Gruppe mit der höchsten Stimmlaustärke. Diese Studiengruppe weist gegenüber den anderen 3 Gruppen die wenigsten Stimm-Handicaps auf (77\%: kein Handicap, 21\%: leichtes Handicap, 1\%: mittelgradiges Handicap).

\subsubsection{Weitere Forschungsansätze}

\subsubsection{Situative Stimmlautstärke}

Anhand dieser Arbeit konnte belegt werden, dass Medizinstudenten im fortgeschrittenen Studienabschnitt signifikant lauter sprechen als Medizinstudenten im ersten Fachsemester und als gleichaltrige Studenten anderer Fachrichtungen. Sozialisationsprozesse im Medizinstudium führen dazu, dass sich Mediziner eine hohe Lautstärke in ihrer Stimme aneignen. Eine laute Stimme unterstützt die Studenten dabei, ihre zukünftige Arztrolle zu verkörpern. Es wäre denkbar gewesen, dass Medizinstudenten beziehungsweise Ärzte nur dann laut sprechen, wenn sie die Arztrolle 
einnehmen. Jedoch wurden die Studenten nicht während der klinischen Arbeit untersucht, sondern sie nahmen in ihrer Freizeit an der Studie teil. Daraus lässt sich schlussfolgern, dass sie sich die laute Stimme auch im Alltag angeeignet haben. Möglicherweise führen Interaktionen mit Patienten und/oder medizinischen Kollegen dazu, dass die Lautstärke der Stimme noch weiter gesteigert wird. Ein Vergleich der Stimmlautstärke ,außerhalb der Arztrolle‘ mit der Stimmlaustärke ,innerhalb der Arztrolle‘ würde diese Fragestellung klären. Dafür könnten Stimmlautstärken von Medizinstudenten/Ärzten zunächst analog zu den Messverfahren der Stimmstudie (Vorlesen, Interview) bestimmt werden. Im nächsten Schritt wären Messungen der Stimmlautstärke in klinischen Situationen erforderlich (beispielsweise bei Anamnesegesprächen oder Patientenvorstellungen). Ein Studiendesign in dieser Art bildet Ansätze für weiterführende Forschung.

\subsubsection{Stimme in verschiedenen ärztlichen Positionen}

Unsere Stimmanalysen beschränkten sich auf Medizinstudenten, folglich Mediziner in ihrer Ausbildung. Während der medizinischen Ausbildung eignen sich die Studenten neben dem umfangreichen Spektrum an Fachwissen auch professionelle Kompetenzen an. Diese werden gleichermaßen zu dem medizinischen Wissen vorausgesetzt. Nach abgeschlossenem Grundstudium treten junge Ärzte in der Regel die Facharztausbildung an. Die Facharztausbildung führt zu einem weiteren Lernprozess, jedoch primär im klinischen Umfeld. Selbst nach abgeschlossener Facharztausbildung ist es für Ärzte unerlässlich, ein Leben lang weiter zu lernen. Lebenslanges Lernen ist notwendig sowohl für die Fachkenntnisse als auch für die sozialen Kompetenzen. Die interpersonellen Anforderungen an die jeweilige Arztrolle sind mannigfaltig und das Rollenverständnis ändert sich. So wie sechs Jahre Grundstudium den Stimmparameter Lautstärke verändern, könnte sich die weiterführende medizinische Karriere ebenfalls auf die Stimmlautstärke auswirken. Verschiedene Entwicklungen sind theoretisch möglich. In aufsteigender Ordnung der klinischen Hierarchie werden auch zunehmende Kompetenzen und Professionalismus erwartet; das gilt sowohl für die Erwartungen der Patienten als auch die der Kollegen. Ein Zuwachs an Professionalismus geht im Medizinstudium offenbar einher mit einer Stimmlautstärkenzunahme. Bedeutet das konsekutiv, dass die Lautstärke im Verlauf der klinischen Karriere weiterhin zunimmt? Diese Hypothese ist kritisch zu hinterfragen, denn eine zu laute Stimme wird beim Gesprächspartner mutmaßlich auch negative Gefühle auslösen. Ab einem gewissen Grad der Stimmlautstärke vermittelt man seinem Gegenüber starke Wut, Ärger und auch Geringschätzung (Costanzo et al. 1969, Siegman et al. 1990). Diese Wirkung hätte schlechten Einfluss auf zwischenmenschliche Kommunikation und würde ArztPatienten-Beziehungen und das Arbeitsklima unter Kollegen belasten. Möglicherweise ließe sich mit dem klinischen, beruflichen Aufstieg wieder ein Rückgang der 
Stimmlautstärke erkennen. Es ist denkbar, dass ab einem gewissen Status die eingenommene Rolle/Position weniger durch nonverbale beziehungsweise paraverbale Mechanismen hervorgehoben werden muss. Ein Vergleich der Stimmlautstärken zwischen Assistenzärzten, Oberärzten und Chefärzten könnte die angeführten Fragestellungen klären. 


\section{Zusammenfassung}

Die medizinsoziologische Forschung konnte belegen, dass das Medizinstudium Ansprüche an die Persönlichkeitsentwicklung der angehenden Ärzte stellt. Eine professionelle Identität hilft den Medizinstudenten die Arztrolle einzunehmen. Das gesellschaftliche Bild des Arztes ist geprägt von Kompetenz, Souveränität und einem daraus resultierenden, sicheren Auftreten. Bisherige Studien konnten nur schwer messbare Parameter aufzeigen, die die Entwicklung der Medizinstudenten in die beschriebene Arztrolle erfassen.

Kommunikation ist ein wichtiger Bestandteil der ärztlichen Tätigkeit - vorrangig in der Arzt-Patienten-Beziehung, aber auch in der Interaktion unter ärztlichen Kollegen und in der Zusammenarbeit von Ärzten mit dem Pflegepersonal. Da kommunikative Fähigkeiten helfen können, die professionelle Identität eines Arztes zu unterstreichen, müssen Medizinstudenten sich in diesem Bereich weiterentwickeln.

Unsere Stimme ist Träger der gesprochenen Sprache. Sie bietet messbare Parameter wie beispielsweise die mittlere Stimmlautstärke oder die mittlere Stimmfrequenz. Für die Stimmstudie war der Parameter Stimmlautstärke von besonderem Interesse, da Menschen mit einer lauten, kräftigen Stimme als durchsetzungsfähig, überzeugend, dominant und selbstbewusst angesehen werden. Solch ein Ansehen deckt sich mit dem gesellschaftlichen Bild des Arztes.

In der vorgelegten Studie wurden Stimmlautstärken von Medizinstudenten im ersten Semester und von Medizinstudenten gegen Ende ihres Studiums verglichen. Analog erfolgte ein Vergleich unter Nichtmedizinstudenten. Es konnte bewiesen werden, dass die Interaktion aus Studiensemester und Studienfach einen hochsignifikanten Einfluss nimmt auf die Stimmlautstärken der Studenten. Medizinstudenten im letzten Studienjahr reden lauter als Medizinstudenten im ersten Semester. Weiterhin konnte belegt werden, dass Medizinstudenten im fortgeschrittenen Studienabschnitt lauter redeten als die soziodemografisch parallelisierte Vergleichsgruppe der Nichtmediziner. Bezogen auf die Nichtmedizinstudenten, sprachen die Nichtmediziner im 9./10.Semester leiser als ihre Kommilitonen/innen im ersten Semester. Mediziner lernen also - anders als Studenten anderer Fakultäten - während des Medizinstudiums ihre Stimmlautstärke zu erhöhen. Dies lässt mutmaßen, dass die Persönlichkeitsentwicklung zu einem gewissen Professionalismus im Medizinstudium eine höhere Rangordnung einnimmt als in anderen Fachgebieten. 


\section{Anhang}

\subsection{Studieninformationen}

\section{GEORG-AUGUST-UNIVERSITÄT GÖTTINGEN}

Bereich Humanmedizin Medizinische Fakultät

\section{UNIVERSITÄTSMEDIZIN \\ GÖTINGEN : UMG}

\section{ABTEILUNG ALLGEMEINMEDIZIN}

Direktor: Univ.-Prof. Dr. med. Michael M. Kochen, MPH, FRCGP

Informationen zur Studie

Humboldtallee 38

D-37073 Göttingen

Tel.: 0551 - 392648

„Veränderung von Parametern der Sprechstimme im Studium“

Telefax: 0551 - 399530

e-mail:whimmel@gwdg.de www.allgemeinmedizin.med.uni-goettingen.de

Dezember 2009

Liebe Kommilitonin,

lieber Kommilitone,

im Rahmen einer Studie möchten wir Näheres über die Stimme und mögliche Stimmbelastungen im Studium und in späteren Sprechberufen erfahren. Daher möchten wir Studierende zu einer Aufnahme ihrer Sprechstimme einladen. Zur Stimmentwicklung und möglichen Stimmbelastungen während des Studiums gibt es bisher wenig Basisdaten. Wir suchen dafür Studierende verschiedener Fachrichtungen, jeweils im 1., $\underline{\text { 9. oder } 10 .}$. Fachsemester.

\section{Was Sie tun müssen}

Wenn Sie sich zur Teilnahme an dieser Studie bereit erklären, müssen Sie lediglich an drei Aufgaben teilnehmen: (1) einen kurzen Text vorlesen und (2) einige Fragen zum Studium beantworten; (3) zum Abschluss bitten wir Sie noch um Beantwortung eines Fragebogens zu persönlichen Einstellungen und Verhaltensweisen. Das Ganze wird etwa 10 bis 15 Minuten dauern und in der Abteilung Allgemeinmedizin in der Humboldtallee 38 stattfinden.

Um Stimmparameter zu messen, wird Ihre Stimme beim Vorlesen und Beantworten der Fragen aufgezeichnet.

Jeder Teilnehmer erhält einen Wertgutschein bei einem Fitnesscenter in Göttingen.

\section{Anonymität, Einverständniserklärung und Datenschutz}

Die Aufzeichnung Ihrer Stimme geschieht anonym, ohne jegliche Speicherung privater Daten. Nach Auswertung der Parameter wird der gesprochene Text selbst gelöscht. Angaben zu Ihrer Person (z. B. Alter, Geschlecht) werden ebenfalls anonym gespeichert und von Ihrem Namen getrennt aufbewahrt. Alle Daten und Auswertungen zu Ihrem Stimmprofil werden ausschließlich in Rahmen dieser Studie verwendet. 
Die Teilnahme an der Studie ist selbstverständlich freiwillig. Sie können jederzeit Ihre Teilnahmeerklärung an der Studie zurückziehen. Es werden dann sämtliche Angaben zu Ihrer Person sofort gelöscht.

\section{Betreuung}

Die Informationen zur Studie haben Sie von Maria Strauss (Doktorandin der Universität Göttingen) erhalten. Sollten Sie noch Fragen haben, so wenden Sie sich doch bitte an mich - am besten per E-mail: stimmstudie@gmx.de - oder an die Verantwortlichen für das Projekt:

Prof. Dr. Wolfgang Himmel, Tel. 39-22648

Prof. Dr. Christiane Kiese-Himmel, Tel. 39-22844

Priv.-Doz. Dr. Martin Scherer, Tel. 39-14227

\section{So finden Sie uns:}
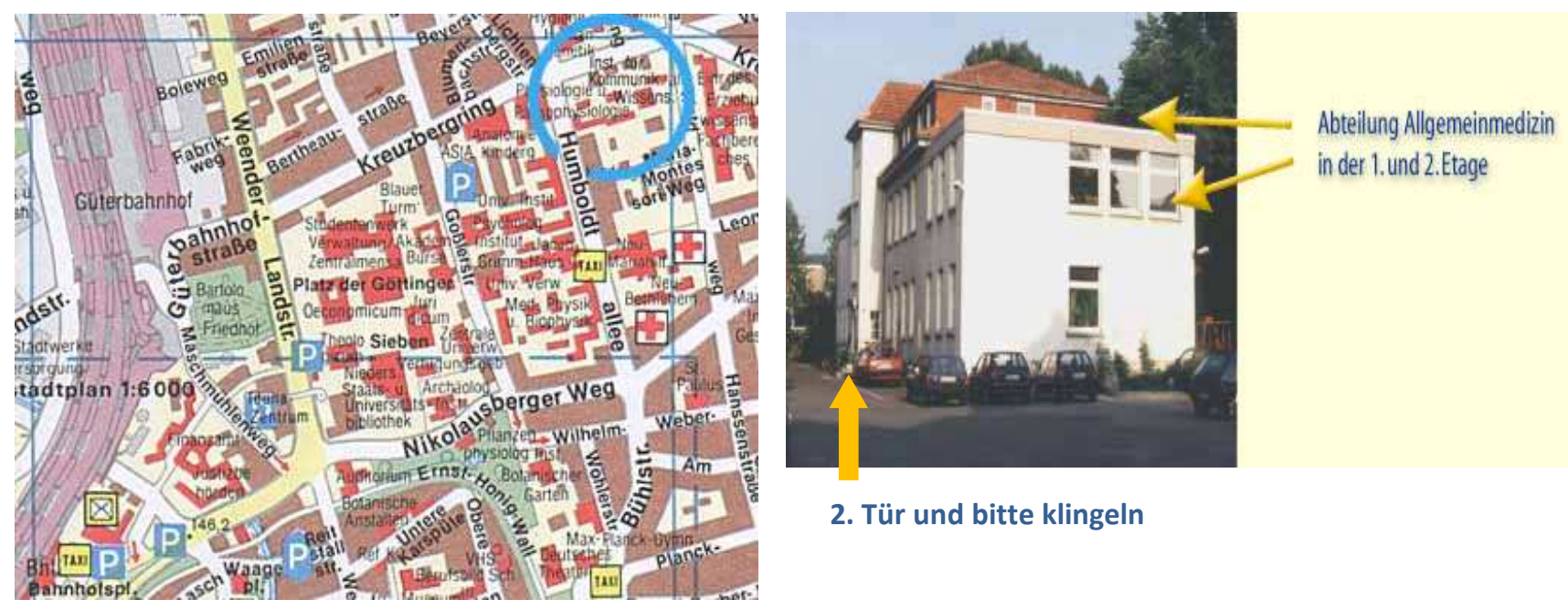

2. Tür und bitte klingeln 


\subsection{Aushang}

GEORG-AUGUST-UNIVERSITÄT GÖTTINGEN

UNIVERSITÄTSMEDIZIN

GÖTTINGEN

UMG

Bereich Humanmedizin Medizinische Fakultät

ABTEILUNG ALLGEMEINMEDIZIN

Direktor: Univ.-Prof. Dr. med. Michael M. Kochen, MPH, FRCGP

\section{Probanden für Studie gesucht!}

Die Stimme des Menschen ist durch die Stimmlippen erzeugter und in den Mund-, Rachen und Nasenraum modulierter Schall. Sie unterliegt im Verlauf des Lebens Veränderungen, zum Beispiel erweitert sich der Stimmumfang.

Die Stimme des Menschen ist ein wichtiges Instrument zum Sprechen. So bedarf es, um miteinander sprachlich kommunizieren zu können, einer

Stimmleistung. Diese Leistung der Sprechstimme wird durch verschiedene Parameter bestimmt und kann gemessen werden.

Anhand von einigen Stimmbeispielen (z. B. Vorlesen eines kurzen Standardtextes; kurzes Gespräch) möchten wir Ihre Stimme akustisch auswerten. Hierzu ist eine apparative Voraussetzung in einem standardisierten Setting erforderlich (z. B. fester Mikrophonabstand), mit der wir Ihre Stimme aufnehmen (und statistisch auswerten). Hierzu suchen wir Studierende verschiedener Fachrichtungen, jeweils im 1., 9. oder 10. Fachsemester.

Die Aufzeichnung Ihrer Stimme geschieht anonym, ohne jegliche Speicherung privater Daten. Das Ganze wird etwa 10 bis 15 Minuten dauern und in der Abteilung Allgemeinmedizin in der Humboldtallee 38 stattfinden.

Jeder Teilnehmer erhält einen Wertgutschein!

Möchten Sie weitere Informationen erhalten, so wenden Sie sich doch bitte per E-Mail an: stimmstudie@gmx.de oder an die Verantwortlichen für das Projekt: Prof. Dr. Wolfgang Himmel (Tel. 39-22648), Prof. Dr. Christiane Kiese-Himmel (Tel. 39-22844), Priv.-Doz. Dr. Martin Scherer (Tel. 39-14227).

Über Ihre Unterstützung an der Studie würden wir uns sehr freuen. Vielen Dank! 


\subsection{Einverständniserklärung}

GEORG-AUGUST-UNIVERSITÄT GÖTTINGEN

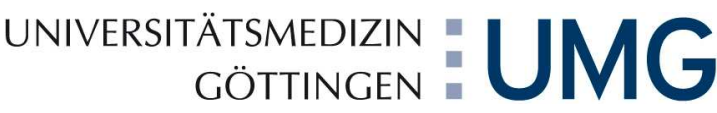

Bereich Humanmedizin Medizinische Fakultät

ABTEILUNG ALLGEMEINMEDIZIN

Direktor: Univ.-Prof. Dr. med. Michael M. Kochen, MPH, FRCGP

\section{Einverständniserklärung}

Humboldtallee 38

D-37073 Göttingen

Tel.: 0551 - 392648

Telefax: 0551 - 399530

e-mail: whimmel@gwdg.de

www.allgemeinmedizin.med.uni-goettingen.de

Dezember 2009

Ich habe die Informationen zur Studie „Veränderung von Parametern der Sprechstimme im Studium“ sorgfältig gelesen und verstanden.

Ich bin damit einverstanden, dass meine Stimme beim Vorlesen eines Standardtextes und bei der Beantwortung von drei Fragen aufgezeichnet wird. Ich bin bereit, die zusätzlichen Fragen zu beantworten.

Ich hatte genügend Zeit, mich zur Teilnahme zu entscheiden. Ich weiß, dass die Teilnahme freiwillig ist. Ich kann jederzeit ohne Angaben von Gründen diese Zustimmung widerrufen, ohne dass Nachteile für mich entstehen. Auch wenn ich nach Stimmaufzeichnung die Teilnahme an der Studie zurückziehe, werden sämtliche bis dahin erhobene bzw. aufgezeichnete Daten gelöscht.

Ich erkläre meine freiwillige Teilnahme an der Studie. Ich weiß, dass der von mir im Rahmen dieser Studie gesprochene Text nicht gespeichert wird. Ich weiß, dass alle im Rahmen dieser Studie erhobenen Daten nur für diese Studie verwendet werden.

Ich habe die Informationsblätter zur Studie erhalten.

- Ich möchte über die Ergebnisse der Stimmaufnahme informiert werden.

E-mail:

Name des Teilnehmers:

Alter (in Jahren):

Ort Datum Unterschrift der Teilnehmerin/des Teilnehmers




\subsection{Standardtext ,Nordwind und Sonne“}

Einst stritten sich Nordwind und Sonne, wer von ihnen beiden wohl der Stärkere wäre, als ein Wanderer, der in einen warmen Mantel gehüllt war, des Weges kam. Sie wurden einig, dass derjenige für den Stärkeren gelten sollte, der den Wanderer zwingen würde, seinen Mantel abzunehmen.

Der Nordwind blies mit aller Macht, aber je mehr er blies, desto fester hüllte sich der Wanderer in seinen Mantel ein. Endlich gab der Nordwind den Kampf auf. Nun erwärmte die Sonne die Luft mit ihren freundlichen Strahlen, und schon nach wenigen Augenblicken zog der Wanderer seinen Mantel aus.

Da musste der Nordwind zugeben, dass die Sonne von innen der Stärkere war. 


\subsection{Stimmfeld (ein Probandenbeispiel)*}

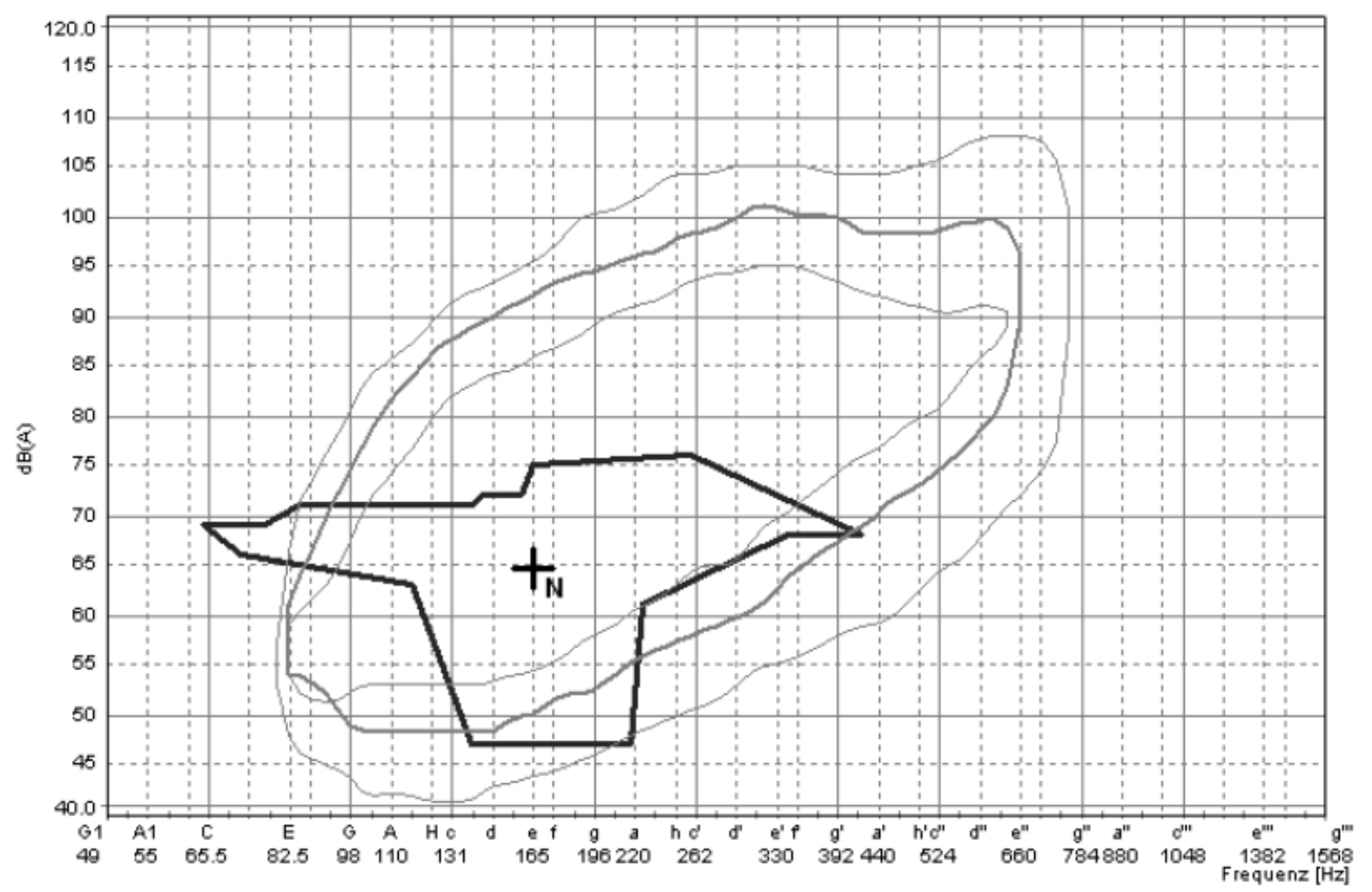

*das Kreuz kennzeichnet die durchschnittliche Stimmlautstärke in dB für eine der beiden Stimmfeldmessungen 


\section{6 lingWAVES Fragebogen VDI}

Anamnese

Fragen
Auswertung

Beruf Frezei normale Interhaituncer

Ich brauche meine Sprechstimme vorwiegend für

Ich brauche meine Singstimme vorwiegend fur

Ich schătze meine Gesprãchigkeit so ein (bitte ankreuzen):

Wie schatzen Sie thre Stimme heute ein?
Anamnese

Fragen
Auswertung

Dies sind Feststellungen, mit denen viele Leute inre Stimme und die Wirkung inrer Stimme auf ihr Leben beschreiben.

Kreuzen Sie die Antwort an, die anzeigt, wie haufig Sie dieselbe Erfahrung machen

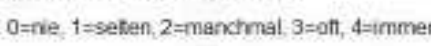

(P) Bevor ich spreche, weiß ich nicht, wie klar meine Stimme klingen wird.

$\begin{array}{lllll}0 & 0 & 2 & 3 & 4\end{array}$

(P) Abends ist meine Stimme schlechter.

$\begin{array}{lllll}0 & 0 & 2 & 3 & 4\end{array}$

(P) Ich habe das Gefühl, dass ich mich anstrengen muss, wenn ich meine Stimme benu...

$\begin{array}{lllll}0 & 0 & 2 & 3 & 4\end{array}$

(E) Ich bin weniger kontaktfreudig wegen meines Stimmproblems.

$\begin{array}{lllll}0 & 0 & 2 & 3 & 4\end{array}$

(E) lch meide großere Gruppen wegen meiner Stimme.

(0) 4 2 2 3 4

(F) Ich fülie mich bei Unterhaltungen wegen meiner Stimme ausgeschiossen.

$\begin{array}{lllll}0 & 0 & 2 & 3 & 4\end{array}$

(F) Anderen fallit es schwer, mich in einem lauten Raum zu verstehen.

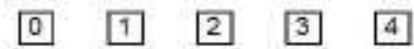

(F) Meine Familie hort mich kaum, wenn ich zuhause nach ihnen rufe.

$\begin{array}{llll}0 & 0 & 2 & 3\end{array}$

(F) Man hört mich wegen meiner Stimme schlecht.

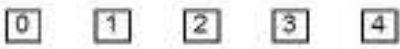

(E) Es ist mir peinlich, wenn Leute mich bitten, etwas zu wiederholen.

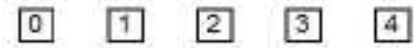

(E) Ich ärgere mich, wenn man mich bittet, etwas zu wiederholen.

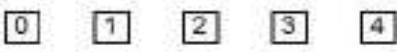

(E) Ich schame mich wegen meines Stimmproblems.

$\begin{array}{llll}0 & 1 & 2 & 3\end{array}$ 


\title{
8.7 Soziodemografische Fragen
}

$\mathrm{Nr}$ :

\begin{abstract}
Soziodemografische Fragen
Geschlecht $\quad \square_{1}$ männl. $\quad \square_{2}$ weibl.

Datum

Lebensalter (in Jahren)

Studienfach

Semesterzahl

Sind Sie derzeit in einer logopädischen Therapie / Sprach- oder Stimmtherapie?

$\square_{0}$ nein $\quad \square_{1}$ ja Wenn ja, weswegen:

Haben Sie jemals eine logopädische Therapie / Sprach- oder Stimmtherapie in Anspruch genommen? $\square_{0}$ nein $\square_{1}$ ja Wenn ja, weswegen:
\end{abstract}

Wann und wie lange (Zahl der Behandlungseinheiten)?

Nehmen Sie derzeit an einem der unten genannten Kurse bzw. an einer ähnlichen Maßnahme teil?

$\begin{array}{lll}\text { Stimmtraining } & \square_{0} \text { nein } & \square_{1} \text { ja } \\ \text { Sprecherziehung } & \square_{0} \text { nein } & \square_{1} \mathrm{ja} \\ \text { Sprechtraining } & \square_{0} \text { nein } & \square_{1} \mathrm{ja} \\ \text { ähnliche Maßnahme? } \square_{0} \text { nein } & \square_{1} \mathrm{ja}\end{array}$

Wenn ja, welche?

Wie würden Sie Ihre Lautstärke beim Sprechen einschätzen?

$\square_{2}$ eher laut $\square_{1}$ eher leise $\square \square_{0}$ normal

Sind Sie in Ihrer Freizeit

Sporttrainer

Lehrender im Unterricht (z. B. Schule, Universität)

Laienschauspieler

$\begin{array}{ll}\square_{0} \text { nein } & \square_{1} \text { ja } \\ \square_{0} \text { nein } & \square_{1} \text { ja } \\ \square_{0} \text { nein } & \square_{1} \text { ja }\end{array}$

Lebt ein Schwerhöriger in Ihrem Umfeld?

$\square_{0}$ nein $\quad \square_{1}$ ja

Wenn ja, wer?

Wurden Sie schon einmal von Freunden / Bekannten / Familienangehörigen darauf hingewiesen, dass sich die Lautstärke Ihrer Stimme verändert hat?

$\square_{0}$ nein $\quad \square_{1}$ ja Wenn ja,

wann?

in welcher Hinsicht? 


\subsection{Fragen aus dem IAF}

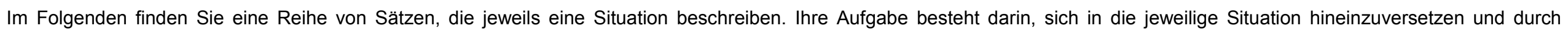

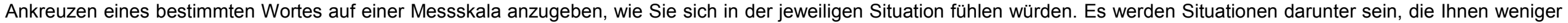

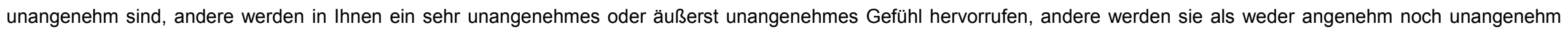
beurteilen.

\section{6: Sie sollen sich am}

nächsten Tag einem psychologischen

Angenehm

Charaktertest unterziehen.

ziemlich

$\square$

12: Wie unangenehm wäre es Ihnen

einem Vorgesetzten zu widersprechen,

wenn Sie sich im Recht fühlten?

19: Wie unangenehm wäre es Ihnen,

sich beim Kellner wegen

schlechter Bedienung zu beschweren?

26: Wie unangenehm wäre es Ihnen,

jemanden, der Sie beleidigte,

zur Rede zu stellen?

35: Wie unangenehm wäre es Ihnen,

einen Vorgesetzten auf einen Fehler

aufmerksam zu machen?

45: Wie unangenehm wäre es Ihnen,

jemanden zur Rede zu stellen,

der sich in einer Warteschlange

vor Sie gedrängelt hat?

\section{Männer:}

Wie unangenehm wäre es Ihnen,

auf einer Gesellschaft eine

unbekannte nette (junge) Dame anzusprechen?

$\square$ ein wenig

$\square$

weder noch

ein wenig

$\square$

$\square$

$\square$

\section{Unangenehm} ziemlich sehr äußerst

$\square \square$

$\square \square$

$\square$

$\square$

$\square$

$\square \quad \square \quad \square$

$\square \quad \square \quad \square$

Wie unangenehm wäre es Ihnen,

auf einer Gesellschaft einen

unbekannten netten (jungen) Mann anzusprechen? $\square$

a




\subsection{Fragen aus dem FPI-R}

Sie finden im Folgenden eine Reihe von Aussagen über bestimmte Verhaltensweisen, Einstellungen und Gewohnheiten. Sie können jede entweder mit „s t i m m t“ oder „s t i m m t $\mathrm{n}$ i c h t" beantworten. Setzen Sie bitte ein Kreuz in die dafür vorgesehene Spalte. Es gibt keine richtigen oder falschen Antworten, weil jeder Mensch das Recht zu eigenen Anschauungen hat. Antworten Sie bitte so, wie es für Sie zutrifft.

Beachten Sie bitte:

- Überlegen Sie nicht erst, welche Antwort vielleicht den besten Eindruck machen könnte, sondern antworten Sie so, wie es für Sie persönlich gilt. Manche Frage kommt Ihnen vielleicht sehr persönlich vor. Bedenken Sie aber, dass Ihre Antworten unbedingt vertraulich behandelt werden.

- Denken Sie nicht lange über einen Satz nach, sondern geben Sie die Antwort, die Ihnen unmittelbar in den Sinn kommt. Natürlich können Sie mit diesen kurzen Fragen nicht alle Besonderheiten berücksichtigt werden. Vielleicht passen deshalb einige nicht gut auf Sie. Kreuzen Sie aber trotzdem immer eine Antwort an, und zwar die, welche noch am ehesten auf Sie zutrifft.

\begin{tabular}{|l|l|l|l|}
\hline Nr. & \multicolumn{1}{|c|}{ stimmt } & $\begin{array}{l}\text { stimmt } \\
\text { nicht }\end{array}$ \\
\hline 12 & $\begin{array}{l}\text { Wenn jemand meinem Freund etwas Böses tut, bin ich } \\
\text { dabei, wenn es heimgezahlt wird }\end{array}$ & \\
\hline 15 & $\begin{array}{l}\text { Ich kann mich erinnern, mal so zornig gewesen zu sein, dass } \\
\text { ich das nächst beste Ding nahm und es zerriss oder } \\
\text { zerschlug }\end{array}$ & $\begin{array}{l}\text { Sind wir in ausgelassener Runde, so überkommt mich oft } \\
\text { eine große Lust zu groben Streichen }\end{array}$ & \\
\hline 20 & $\begin{array}{l}\text { Ich male mir manchmal aus, wie übel es denen eigentlich } \\
\text { ergehen müsste, die mir Unrecht tun }\end{array}$ & & \\
\hline 37 & $\begin{array}{l}\text { Als Kind habe ich manchmal ganz gerne anderen die Arme } \\
\text { umgedreht, an Haaren gezogen, ein Bein gestellt usw. }\end{array}$ & & \\
\hline 40 & $\begin{array}{l}\text { Es macht mir Spaß, anderen Fehler nachzuweisen } \\
\text { menn ich Zuflucht zu körperlicher Gewalt nehmen muss, um } \\
50\end{array}$ & $\begin{array}{l}\text { Wechte zu verteidigen, so tue ich es } \\
\text { Wenn mich jemand anschreit, schreie ich zurück }\end{array}$ & \\
\hline 56 & $\begin{array}{l}\text { Wenn ich wirklich wütend werde, bin ich in der Lage, } \\
\text { jemanden eine runterzuhauen }\end{array}$ & & \\
\hline 90 & $\begin{array}{l}\text { Einem Menschen, der mich schlecht behandelt oder beleidigt } \\
\text { hat, wünsche ich eine harte Strafe }\end{array}$ & & \\
\hline 132 & Lieber bis zum Äußersten gehen als feige sein & & \\
\hline 137 & $\begin{array}{l}\text { Es gab Leute, die mich so ärgerten, dass es zu einer } \\
\text { handfesten Auseinandersetzung kam }\end{array}$ & & \\
\hline
\end{tabular}




\subsection{HADS-D}

Bitte beantworten Sie jede der folgenden Fragen so, wie es für Sie persönlich in der letzten Woche am ehesten zutraf. Machen sie bitte nur ein Kreuz pro Frage und lassen Sie bitte keine Frage aus! Überlegen Sie bitte nicht lange, sondern wählen Sie die Antwort aus, die Ihnen auf Anhieb am zutreffendsten erscheint!

\begin{tabular}{|c|c|}
\hline $\begin{array}{l}\text { 1) Ich fühle mich angespannt oder über- } \\
\text { reizt } \\
\square \text { meistens } \\
\square \text { oft } \\
\square \text { gelegentlich } \\
\square \text { überhaupt nicht }\end{array}$ & $\begin{array}{l}\text { 8) Ich fühle mich in meinen Aktivitäten } \\
\text { gebremst } \\
\square \text { fast immer } \\
\square \text { sehr oft } \\
\square \text { manchmal } \\
\square \text { überhaupt nicht }\end{array}$ \\
\hline $\begin{array}{l}\text { 2) Ich kann mich heute noch so freuen wie } \\
\text { früher } \\
\square \text { ganz genau so } \\
\square \text { nicht ganz so sehr } \\
\square \text { nur noch ein wenig } \\
\square \text { kaum oder gar nicht }\end{array}$ & $\begin{array}{l}\text { 9) Ich habe manchmal ein ängstliches Ge- } \\
\text { fühl in der Magengegend } \\
\square \text { überhaupt nicht } \\
\square \text { gelegentlich } \\
\square \text { ziemlich oft } \\
\square \text { sehr oft }\end{array}$ \\
\hline $\begin{array}{l}\text { 3) Mich überkommt eine schreckliche Vor- } \\
\text { ahnung, dass etwas Schreckliches } \\
\text { passieren könnte } \\
\square \text { ja, sehr stark } \\
\square \text { ja, aber nicht zu stark } \\
\square \text { etwas, aber es macht mir keine Sorgen } \\
\square \text { überhaupt nicht }\end{array}$ & $\begin{array}{l}\text { 10) Ich habe das Interesse an meiner } \\
\text { äußeren Erscheinung verloren } \\
\square \text { ja, das stimmt genau } \\
\square \text { ich kümmere mich nicht so darum, wie } \\
\text { ich sollte } \\
\square \text { evtl. kümmere ich mich zuwenig darum } \\
\square \text { ich kümmere mich so viel darum wie } \\
\text { immer }\end{array}$ \\
\hline $\begin{array}{l}\text { 4) Ich kann lachen und die lustigen Dinge } \\
\text { sehen } \\
\square \text { ja, so viel wie immer } \\
\square \text { nicht mehr ganz so viel } \\
\square \text { inzwischen viel weniger } \\
\square \text { überhaupt nicht }\end{array}$ & $\begin{array}{l}\text { 11) Ich fühle mich rastlos, muss immer in } \\
\text { Bewegung sein } \\
\square \text { Ja, tatsächlich sehr } \\
\square \text { ziemlich } \\
\square \text { nicht sehr } \\
\square \text { überhaupt nicht }\end{array}$ \\
\hline $\begin{array}{l}\text { 5) Mir gehen beunruhigende Dinge durch } \\
\text { den Kopf } \\
\square \text { einen Großteil der Zeit } \\
\square \text { verhältnismäßig oft } \\
\square \text { von Zeit zu Zeit, aber nicht zu oft } \\
\square \text { nur gelegentlich / nie }\end{array}$ & $\begin{array}{l}\text { 12) Ich blicke mit Freude in die Zukunft } \\
\square \text { ja, sehr } \\
\square \text { eher weniger als früher } \\
\square \text { viel weniger als früher } \\
\square \text { kaum bis gar nicht }\end{array}$ \\
\hline $\begin{array}{l}\text { 6) Ich fühle mich glücklich } \\
\square \text { überhaupt nicht } \\
\square \text { selten } \\
\square \text { manchmal } \\
\square \text { nie }\end{array}$ & $\begin{array}{l}\text { 13) Mich überkommt plötzlich ein panik- } \\
\text { artiger Zustand } \\
\square \text { ja, tatsächlich sehr oft } \\
\square \text { ziemlich oft } \\
\square \text { nicht sehr oft } \\
\square \text { überhaupt nicht } \\
\end{array}$ \\
\hline $\begin{array}{l}\text { 7) Ich kann behaglich dasitzen und mich } \\
\text { entspannen } \\
\square \text { Ja, natürlich } \\
\square \text { gewöhnlich schon } \\
\square \text { nicht oft } \\
\square \text { überhaupt nicht }\end{array}$ & $\begin{array}{l}\text { 14) Ich kann mich an einem guten Buch, } \\
\text { einer Radio- oder Fernsehsendung freuen } \\
\square \text { oft } \\
\square \text { manchmal } \\
\square \text { eher selten } \\
\square \text { sehr selten }\end{array}$ \\
\hline
\end{tabular}




\section{Literaturverzeichnis}

Amon I: Die Macht der Stimme. Persönlichkeit durch Klang, Volumen und Dynamik. 4.Auflage; Redline Wirtschaft Finanzbuch Verlag GmbH, München 2008

Angelillo M, di Maio G, Costa G, Angelillo N, Barillari U (2009): Prevalence of occupational voice disorders in teachers. J Prev Med Hyg $\underline{50}$, 26-32

Bargel T, Ramm M: Das Studium der Medizin. Erfahrungen, Probleme und Forderungen aus studentischer Sicht. Hrsg. v. Bundesministerium für Bildung und Wissenschaft, Bonn 1993

Barrett RJ (1988): Clinical writing and the documentary construction of schizophrenia. Cult Med Psychiatry 12, 265-299

Bazil V, Piwinger M: Über die Funktion der Stimme in der Kommunikation; in: Handbuch Kommunikationsmanagement; hrsg. v. Bentele G, Piwinger M, Schönborn G; Luchterhand-Fachverlag, Neuwied 2009, 1-22; Unter: http://www.piwinger.de/aktuell/FunktionDerStimelnDerKommunikation.html (zuletzt abgerufen am 08.01.2012)

Becker P: Interaktions-Angst-Fragebogen. Manual., 3.Auflage; Beltz Test GmbH, Göttingen 1997

Brazeau CMLR, Schroeder R, Rovi S, Boyd Linda (2010): Relationships between medical student burnout, empathy, and professionalism climate. Acad Med $\underline{85}$, S33-S36

Bründler $P$, Bürgisser D, Lämmli D, Bornand J: Einführung in die Psychologie und Pädagogik. Compendio Bildungsmedien, Zürich 2004

Costanzo FS, Markel NN, Costanzo PR (1969): Voice quality profile and perceived emotion. J Couns Psychol 16, 267-270

De Ceballos AG, Carvalho FM, de Araújo TM, Dos Reis EJ (2011): Auditory vocal analysis and factors associated with voice disorders among teachers. Rev Bras Epidemiol 14, 285-295

Dornan T, Bundy C (2004): Learning in practise. What can experience add to early medical education? Consensus survey. BMJ $\underline{329}, 1-6$

Dyrbye LN, Thomas MR, Shanafelt TD (2006): Systematic review of depression, anxiety, and other indicators of psychological distress among U.S. and Canadian medical students. Acad Med 81, 354-373 
Dyrbye LN, Thomas MR, Eacker A, Harper W, Massie FS Jr, Power DV, Huschka M, Novotny PJ, Sloan JA, Shanafelt TD (2007): Race, ethnicity, and medical student well-being in the United States. Arch Intern Med 167, 2103-2109

Dyrbye LN, Thomas MR, Massie FS, Power DV, Eacker A, Harper W, Durning S, Moutier C, Szydlo DW, Novotny PJ (2008): Burnout and suicidal ideation among U.S. medical students. Ann Intern Med 149, 334-341

Dyrbye LN, Thomas MR, Power DV, Durning S, Moutier C, Massie SF, Harper W, Eacker A, Szydlo DW, Jeff A (2010): Burnout and serious thoughts of dropping out of medical school: a multi-institutional study. Acad Med $\underline{85}, 94-102$

Dyrbye LN, Harper W, Durning SJ, Moutier C, Thomas MR, Massie FS Jr, Eacker A, Power DV, Szydlo DW, Sloan JA (2011): Patterns of distress in US medical students. Med Teach $\underline{33}, 834-839$

Eisler RM, Miller PM, Hersen M (1973): Components of assertive behaviour. J Clin Psychol 29, 295-299

Fahrenberg J, Hampel R, Selg H: Das Freiburger Persönlichkeitsinventar-R. Manual., 7. Auflage; Hogrefe Verlag für Psychologie, Göttingen 2001

Faller $\mathrm{H}$, Lang $\mathrm{H}$ : Medizinische Psychologie und Soziologie. 2. Auflage; Springer Medizin Verlag, Heidelberg 2006

Good BJ: Medicine, Rationality, and Experience: An Anthropological Perspective. Cambridge University Press, Cambridge 1994

Grassegger H: Phonetik. Phonologie. 4.Auflage; in: BWT Basiswissen Therapie; hrsg. v. Tesak J; Schultz-Kirchner Verlag, Idstein 2010

Grimm H, Engelkamp J: Sprachpsychologie. Handbuch und Lexikon der Psycholinguistik. Erich Schmidt Verlag, Berlin 1981

Gühring W, Barth J: Anatomie; in: Grundwissen für Zahntechniker, Band 3; hrsg. v. Neuer Merkur Verlag, München 1992

Haber RJ, Lingard L (2001): Learning oral presentation skills. A rhetorical analysis with pedagogical and professional implications. J Gen Intern Med 16, 308-314

Hafferty FW (1998): Beyond curriculum reform: Confronting medicine's hidden curriculum. Acad Med $\underline{73}, 403-407$

Harrigan JA, Gramata JF, Lucic KS, Margolis C (1989): It's how you say it: Physicians' vocal behavior. Soc Sci Med $\underline{28}, 87-92$

Hautzinger M: Kognitive Verhaltenstherapie bei Depressionen. 6.Auflage; Verlagsgruppe Beltz, Weinheim 2003 
Hays RB, Jolly BC, Caldon LJM, McCrorie P, McAvoy PA, McManus IC, Rethans JJ (2002): Is insight important? Measuring capacity to change performance. Med Educ $\underline{36}, 965-971$

Heim E, Knapp PH, Vachon L., Globus G., Nemetz SJ (1968): Emotion, breathing and speech. J Psychosom Res 12, 261-274

Herrmann-Lingen C, Buss U, Snaith RP: Hospital Anxiety and Depression ScaleDeutsche Version. 2.Auflage; Hans Huber Verlag, Bern 2005

Hosse R: Arbeitsmedizin und Sozialmedizin; in: Exaplan. Das Kompendium der klinischen Medizin, Band 2; hrsg. v. Emminger H und Kia T; Urban \& Fischer Verlag, München 2008, 2419-2498

Hottel TL, Hardigan PC (2005): Improvement in the interpersonal communication skills of dental students. J Dent Educ $\underline{69}, 281-284$

International Phonetic Association: Handbook of the International Phonetic Association. A Guide to the Use of the International Phonetic Alphabet. Cambridge University Press, Cambridge 1999

Irvine D (1999): The performance of doctors: new professionalism. Lancet $\underline{353}, 1174-$ 1177

Jadoon NA, Yaqoob R, Raza A, Shehzad MA, Choudhry ZS (2010): Anxiety and depression among medical students: A cross-sectional study. J Pak Med Assoc $\underline{60}, 699-702$

Kimble CE, Musgrove JI (1988): Dominance in arguing mixed-sex dyads: visual dominance patterns, talking time, and speech loudness. J Res Pers 2ㅡ, 1-16

Kimble CE, Seidel SD (1991): Vocal signs of confidence. J Nonverbal Behav 15, 99-105

Kjeldstadli K, Tyssen R, Finset A, Hem E, Gude T, Gronvold NT, Ekeberg O, Vaglum P (2006): Life satisfaction and resilience in medical school--a six-year longitudinal, nationwide and comparative study. BMC Med Educ 19, 6:48, unter: http://www.biomedcentral.com/1472-6920/8/46/ (zuletzt abgerufen am 01.01.2012)

Kötter T, Tautphäus Y, Scherer M, Voltmer E (2011): Was hält die Hausärzte von morgen gesund? Luebeck University Students Trial [LUST]. Vorstellung des Studienprotokolls. Universität zu Lübeck.

Krupat E, Yeager CM, Putnam S (2000): Patient role orientations, doctor-patient fit and visit satisfaction. Psychol Health $\underline{15}, 707-719$

Kühn S, Platte I, Wottawa H: Psychologische Theorien für Unternehmen. Vandenhoeck \& Ruprecht, Göttingen 2006 
Lane H, Tranel B (1971): The lombard sign and the role of hearing in speech. J Speech Hear Res 14, 677-709

Leo T, Eagen K (2008): Professionalism Education: The medical student response. Perspect Biol Med $\underline{51}, 508-516$

Lieb K, Frauenknecht S, Brunnhuber S: Intensivkurs Psychiatrie und Psychotherapie. 6.Auflage; Urban und Fischer Verlag, München 2008

Lingard L, Garwood K, Schryer CF, Spafford MM (2003): A certain art of uncertainty: case presentation and the development of professional identity. Soc Sci Med $\underline{56}$, 603-616

lingWAVES Recorder + Stimmstörungsindex Handbuch. Version 2.5; WEVOS WEVOSYS - Germany 2005

Metzig W, Schuster M: Prüfungsangst und Lampenfieber. 3.Auflage; Springer Verlag, Berlin 2006

Moosbrugger $\mathrm{H}$, Kelava A: Testtheorie und Fragebogenkonstruktion. Springer Medizin Verlag, Heidelberg 2008

Müller K: Kenntnisse und Einstellungen klinisch tätiger Ärzte zum Patienten-Gespräch. Eine Untersuchung zum ärztlichen Kommunikationsverhalten. Phil. Diss. Berlin 2004

Nawka T, Wiesmann U, Gonnermann U (2003): Validierung des Voice Handicap Index (VHI) in der deutschen Fassung. HNO $\underline{51}, 921-929$

Nawka T, Franke I, Galkin E (2006): Objektive Messverfahren in der Stimmdiagnostik. Forum Logopädie $\underline{4}, 14-21$

Nietzsche F: Menschliches, Allzumenschliches 1878 (Sämtliche Werke / Friedrich Nietzsche, Kritische Studienausgabe, Band 2); hrsg. v. Colli G und Montinari M; Dt. Taschenbuch-Verlag de Gruyter, München 1999

Pekkarinen E, Viljanen V (1991): Acoustic conditions for speech communication in classrooms. Scand Audiol 20, 257-263

Péréz Álvarez JC, Hacki, T (2000): Norm-Stimmparameter beim Standardlesetext; in: Aktuelle phoniatrisch-pädaudiologische Aspekte 1999/2000, Band 7; hrsg. v. Gross M; Median-Verlag, Heidelberg 2000, 78-81

Philipp S: Kommunikation und Interaktion; in: Lehrbuch Medizinische Psychologie und Medizinische Soziologie; hrsg. v. Strauß B, Berger U, von Troschke J, Brähler E; Hogrefe Verlag, Göttingen 2004, 341-352

Piok M: Gesprochene Sprache und literarischer Text. Lit Verlag, Berlin 2011 
Pitkala KH, Mantyranta T (2003): Professional socialization revised: medical students' own conceptions related to adoption of the future physician`s role-a qualitative study. Med Teach $\underline{25}, 155-160$

Plewnia CG: Wandel der Arztideale. Entwicklungen in Abhängigkeit von der Dauer der Berufstätigkeit (Internationale Hochschulschriften, Band 302); hrsg. v. Waxmann Verlag GmbH, Münster 1999

Prost W: Manipulation und Überzeugungskunst. Wie Sie andere gewinnen und sich vor Fremdsteuerung schützen. Gabler Fachverlag, Wiesbaden 2009

Radcliffe C, Lester H (2003): Perceived stress during undergraduate medical training: a qualitative study. Med Educ 37, 32-38

Ramig LO, Gray S, Baker K, Corbin-Lewis K, Bruder E, Luschei E, Smith HCM (2001): The aging voice: a review, treatment data and familial and genetic perspectives. Folia Phoniatr Logop $\underline{53}$, 252-265

Raz AE, Fadlon J (2006): "We came to talk with the people behind the disease:" Communication and control in medical education. Cult Med Psychiatry 30, 55-75

Russell A, Oates J, Greenwood KM (1998): Prevalence of voice problems in teachers. J Voice $\underline{12}$, 467-479

Sala E, Viljanen V (1995): Improvement of acoustic conditions for speech communication in classrooms. Appl Acoust 45, 81-91

Santen SA, Holt DB, Kemp JD, Hemphill RR (2010): Burnout in medical students: examining the prevalence and associated factors. South Med J 103, 758-763

Scherer KR (1978): Personality inference from voice quality: the loud voice of extroversion. Eur J Soc Psychol $\underline{8}$, 467-487

Scherer M, Himmel W, Stanske B, Scherer F, Koschak J, Kochen MM, HerrmannLingen C. (2007): Psychological distress in primary care patients with heart failure: A longitudinal study. Br J Gen Pract $\underline{57}$, 801-807

Schmid Mast M, Hall J, Roter D (2008): Caring and dominance affect participants` perceptions and behaviors during a virtual medical visit. J Gen Intern Med $\underline{23}$, 523-527

Schneider B, Bigenzahn W: Stimmdiagnostik. Ein Leitfaden für die Praxis. Springer Verlag, Wien 2007

Schnell R, Hill PB, Esser E: Methoden der empirischen Sozialforschung. 8.Auflage; Oldenbourg Wissenschaftsverlag, München 2008 
Scholl A: Die Befragung. 2. Auflage; UVK Verlagsgesellschaft, Konstanz 2009

Schultz-Coulon HJ, Asche S (1988): Das „Normstimmfeld“ - ein Vorschlag. Sprache Stimme - Gehör $\underline{12}, 5-8$

Schwenk TL, Davis L, Winsatt LA (2010): Depression, stigma, and suicidal Ideation in medical students. JAMA $\underline{304}, 1181-1190$

Siegman AW, Anderson RA, Berger T (1990): The angry voice: Its effects on the experience of anger and cardiovascular reactivity. Psychosom Med $\underline{52}, 631-643$

Simberg S, Sala E, Vehmas K, Laine A (2005): Changes in the prevalence of vocal symptoms among teachers during a twelve-year period. J Voice $\underline{19}, 95-102$

Södersten M, Ternström S, Bohman M (2005): Loud speech in realistic environmental noise: phonetogram data, perceptual voice quality, subjective ratings, and gender differences in healthy speakers. J Voice $\underline{19}$, 29-46

Spornitz UM: Anatomie und Physiologie. Lehrbuch und Atlas für Pflege- und Gesundheitsfachberufe. 4.Auflage; Springer Medizin Verlag, Heidelberg 2004

Stern D, Papadakis M (2006): The developing physician-becoming a professional. N Engl J Med 355, 1794-1799

Svec JG, Granqvist S (2010): Guidelines for selecting microphones for human voice production research. Am J Speech Lang Pathol 19, 356-368

Titze IR (1999): Toward occupational safety criteria for vocalization. Logoped Phoniatr Vocol 24, 49-54

Verdolini K, Ramig LO (2001): Review: occupational risks for voice problems. Logoped Phoniatr Vocol $\underline{26}$, 37-46

Vilkman E (2000): Voice problems at work: a challenge for occupational safety and health arrangements. Folia Phoniatr Logop $\underline{52}, 120-125$

Wendler J, Seidner W, Eysholdt U: Lehrbuch der Phoniatrie und Pädaudiologie. 4.Auflage, Georg Thieme Verlag, Stuttgart 2005

Zick A, Granieri M, Makoul G (2007): First-year medical students' assessment of their own communication skills. Patient Educ Couns $\underline{68}, 161-166$

Zraick RI, Marshall W, Smith-Olinde L, Montague JC (2004): The effect of task on determination of habitual loudness. J Voice 18 , 176-182 


\section{Lebenslauf}

Am 06.01.1984 wurde ich in Leinefelde als Tochter von Petra Strauß, Sachbearbeiterin für den Bereich Kultur, und Peter Strauß, Musikschulpädagoge, geboren. Ich bin verheiratet und habe keine Kinder.

Ich besuchte von 1990 bis 1994 die Johann Carl Fuhlrott-Grundschule und von 1994 bis 1995 das Gottfried Wilhelm Leibniz-Gymnasium in Leinefelde. Nach einem Umzug wechselte ich 1995 auf das Johann Georg Lingemann-Gymnasium in Heiligenstadt. Hier bestand ich meine Abiturprüfung im Jahr 2002.

Zum Wintersemester 2003/2004 nahm ich das Humanmedizinstudium an der GeorgAugust-Universität in Göttingen auf. Am 17. März 2006 legte ich den Ersten Abschnitt der Ärztlichen Prüfung ab. Während meines klinischen Studienabschnitts famulierte ich in den Fächern Allgemeinchirurgie, Unfallchirurgie und Gynäkologie.

Das erste Tertial meines Praktischen Jahres absolvierte ich in der Abteilung Chirurgie im Evangelischen Krankenhaus Weende in Göttingen. Die Schwerpunkte lagen in den Bereichen Viszeralchirurgie, Unfallchirurgie/Orthopädie sowie der plastischrekonstruktiven Chirurgie. Im Anschluss durchlief ich das zweite Tertial in meinem Wahlfach Urologie im Universitätsklinikum Göttingen. Im letzten Tertial des Praktischen Jahres war ich für das Fach Innere Medizin erneut im Evangelischen Krankenhaus Weende in Göttingen tätig. Hier wählte ich die Schwerpunkte Geriatrie und Zentrale Notaufnahme.

Den Zweiten Abschnitt der Ärztlichen Prüfung bestand ich am 8. Dezember 2010.

Seit dem 1. September 2011 arbeite ich als Assistenzärztin in der Abteilung Chirurgie im Krankenhaus Neu-Bethlehem in Göttingen. 